\title{
Multi-agent Control and Optimization under Uncertainty
}

\author{
A Dissertation \\ Presented to \\ the faculty of the School of Engineering and Applied Science \\ University of Virginia
}

\author{
in partial fulfillment \\ of the requirements for the degree \\ Doctor of Philosophy \\ by
}

Shi Pu

August

2016 
APPROVAL SHEET

The dissertation

is submitted in partial fulfillment of the requirements

for the degree of

Doctor of Philosophy

shipu

AUTHOR

The dissertation has been read and approved by the examining committee:

Quanquan Gu

Advisor

Alfredo Garcia

Stephen Patek

Zongli Lin

\section{Gerard Learmonth}

Accepted for the School of Engineering and Applied Science:

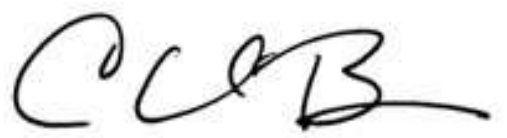

Craig H. Benson, Dean, School of Engineering and Applied Science

August

2016 


\section{UNIVERSITY OF VIRGINIA}

Department of Systems \& Information EngineERING

Dissertation in Partial Fulfillment

of the Requirements for the Degree of

Doctor of Philosophy

\section{Multi-agent Control and Optimization under Uncertainty}

Author:

Supervisors:

Shi PU

Dr. Alfredo Garcia

Dr. Quanquan Gu

August, 2016 


\begin{abstract}
Many natural and engineering systems can be characterized as a collection of interacting agents each having access to local information, making local decisions, having local interactions with neighbors, and seeking to optimize local objectives. The analysis and design of such systems falls under the broader framework of "multi-agent control and optimization". Here depending on the context, "agents" could be animals, autonomous vehicles, power plants, etc. Multi-agent systems have the potential for solving problems that are difficult or impossible for an individual agent or monolithic system to solve. However, the complexity associated with a potentially large number of interacting agents brings about challenging control and optimization problems for system designers and coordinators. Such difficulty is often enhanced by the presence of noise/uncertainty that is pervasive in both biological and engineering systems.

In this dissertation, we focus on the analysis and design of several multi-agent control/optimization algorithms for various problems under uncertainty. Swarming, flocking, schooling and other aggregations of organisms in groups have been studied extensively in biology (see $[64,65,74]$ ). Organisms in swarms can exploit several advantages of staying close to each other for more effective foraging. For example, in [38] Grünbaum explains how social foragers more successfully perform chemotaxis over noisy gradients than individuals. Such biological advantage is also demonstrated in Passino [76] by modeling the behavior of E. coli and M. xanthus bacteria. In our first line of work, we develop a mathematical model for analyzing the benefits of social foraging in a noisy environment. We identify conditions
\end{abstract}


on the nutrient profile ensuring that local agent actions will lead to cohesive foraging. For convex, smooth nutrient profiles we formalize the way in which swarming for social foraging is better at handling the effects of noise when compared to the average of individual foraging strategies. Under a swarming discipline, observational noise realizations that induce trajectories differing too much from the group average are likely to be discarded because of each individual's need to maintain cohesion. As a result, the group trajectories are less affected by noise. Simulation experiments indicate that our theoretical results are also robust to inter-agent communication constraints and non-convex nutrient profiles.

The above results suggest that swarming-like approaches for the control and/or optimization of networked agents may provide an additional level of robustness. This is precisely the gist of our second line of work in which we consider a distributed computing algorithmic scheme for stochastic optimization which relies on modest communication requirements amongst processors and most importantly, does not require synchronization. Specifically, we analyze a scheme with $N>1$ independent threads each implementing a stochastic gradient algorithm. The threads are coupled via a perturbation of the gradient (with attractive and repulsive forces) in a similar manner to mathematical models of flocking. We show that a flocking-like approach for distributed stochastic optimization provides a noise reduction effect similar to that of a centralized stochastic gradient algorithm based upon the average of $N$ gradient samples at each step. When the overhead related to the time needed to gather $N$ samples and synchronization is not negligible, the flocking implementation outperforms the centralized algorithm.

In our last line of work, we consider one of the most important multi-agent systems in any modern economy: the electric power infrastructure. Specifically, we consider the problem of designing the rules by which market dispatch and payment to participants are gradually adjusted while taking into account network and reliability constraints so as to ensure the market clears with an efficient outcome. We propose a class of iterative mechanisms and show this class exhibits many desirable properties (approximately): incentive compatibility, efficiency, individual rationality and (weak) budget balance. In addition, we analyze an iterative 
mechanism for stochastic market clearing, a pressing need given the increasing penetration of highly intermittent renewable generation technologies. In this case, the marginal cost of adjustments may only be estimated with some error. We show that truthful reporting is a Nash equilibrium and the resulting dispatch converges almost surely to the efficient dispatch.

Keywords: Multi-agent systems, collective animal behavior, swarming, flocking, social foraging, noise reduction, distributed stochastic optimization, electricity markets, mechanism design 
To my family. 


\section{Acknowledgments}

First and foremost, I want to express my sincere gratitude to my advisor Dr. Alfredo Garcia for guiding me through the graduate study in UVa. I am very lucky to have Prof. Garcia as my advisor from the very beginning of this journey. Under his direction, I gradually learned how to do research as a $\mathrm{PhD}$ student. Prof. Garcia is very kind and easy-going. We have met and talked a lot over the past four years, and our conversation topic includes everything from research to daily life. At times when I feel frustrated, Prof. Garcia always encourages me and cheers me up. In my eyes he is not only a good mentor but also a great friend. This dissertation is impossible without him.

Next, I want to thank Prof. Gerard Learmonth, Prof. Quanquan Gu, Prof. Stephen Patek and Prof. Zongli Lin for serving in my advisory committee. Their advices and feedbacks helped me improve the dissertation.

I also want to thank my fellow Yue Sun who helped me a lot in my graduate study and life in the US, especially when I just came to UVa. Moreover, I feel grateful to my friends Shuang Chen and Yu Sheng for their valuable suggestions on the dissertation.

I am very fortunate to meet my girlfriend Yan Guo here in UVa. Because of her, my life has become so much more interesting and colorful. She gives me a lot of energy and inspiration for my research as well.

Lastly, I want to thank my parents Jiaying Pu and Shiqin Pan for their love and support. Words are just not enough to express my gratitude to them. 


\section{TABLE OF CONTENTS}

$\begin{array}{ll}\text { Abstract } & \text { i }\end{array}$

Dedication $\quad$ iv

$\begin{array}{ll}\text { Acknowledgments } & \text { V }\end{array}$

List of Figures $\quad$ x

1 Overview 1

1.1 Main Contributions . . . . . . . . . . . . . . . . . . . 5

1.2 Organization of the Dissertation . . . . . . . . . . . . . 6

2 Background $\quad 8$

2.1 Swarming . . . . . . . . . . . . . . . . . . . . 8

2.1.1 Swarming in Biology and Physics ............. 8

2.1.2 Swarming in Engineering . . . . . . . . . . . . . . . . 11

2.2 Stochastic Optimization ......................... 14

2.3 Mechanism Design for Electricity Markets . . . . . . . . . . . . . . . . 15

3 Noise Reduction by Swarming in Social Foraging $\quad 18$

3.1 Introduction . . . . . . . . . . . . . . . . . . . . . . 18

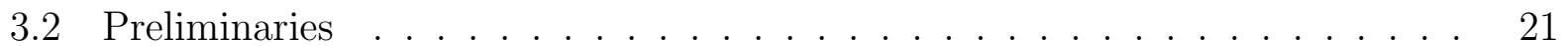


3.3 The Swarm Model . . . . . . . . . . . . . . . . . . . . . . . . 22

3.4 Swarm Cohesion Analysis _. . . . . . . . . . . . . . . . 23

3.4.1 Preliminaries: Macro-micro Decomposition . . . . . . . . . . . . 23

3.4 .2 Swarm Cohesion . . . . . . . . . . . . . . . . . 25

3.5 Social Foraging Properties _ . . . . . . . . . . . . . . . . . . . . . . 32

3.5 .1 Noise Reduction Property _ . . . . . . . . . . . . . . . . 35

3.6 Simulation Examples . . . . . . . . . . . . . . . . . . . . . . . . . . . . 37

3.6 .1 Quadratic Resource Profile . . . . . . . . . . . . . . . . . . 37

$3.6 .2 \quad$ Non-convex Resource Profile . . . . . . . . . . . . . . . . . . . . . . . 39

3.6 .3 Incomplete Graph . . . . . . . . . . . . . . . . . . . . . . 41

3.7 Conclusions of This Chapter . . . . . . . . . . . . . . . . 41

4 A Flocking-based Approach for Distributed Stochastic Optimization 43

4.1 Introduction . . . . . . . . . . . . . . . . . . . . . . . 44

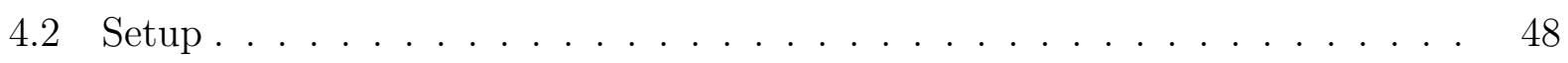

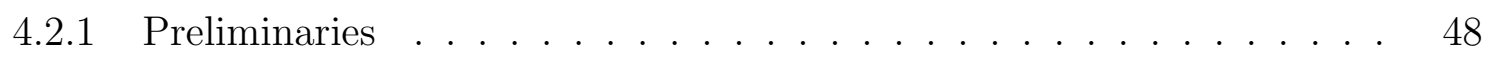

4.2 .2 Problem Statement . . . . . . . . . . . . . . . . . . . . 48

4.3 Analysis . . . . . . . . . . . . . . . . . . . 52

4.3 .1 Preliminaries . . . . . . . . . . . . . . . . . . . 52

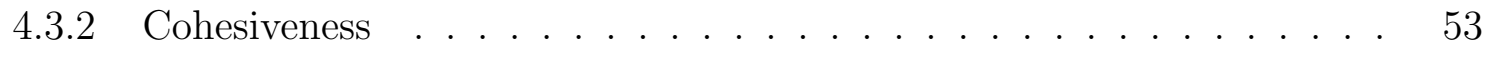

4.4 Noise Reduction in Convex Optimization . . . . . . . . . . . . . . . . . 54

4.4.1 Real-time Performance Comparison . . . . . . . . . . . . . . 57

4.5 Application to Non-Convex Optimization . . . . . . . . . . . . . . 59

$4.5 .1 \quad$ Simulation Examples . . . . . . . . . . . . . . . . . . . . 59

4.5 .2 Asymptotic Noise Reduction . . . . . . . . . . . . . . . . 61

4.6 Conclusions of This Chapter . . . . . . . . . . . . . . . . 63

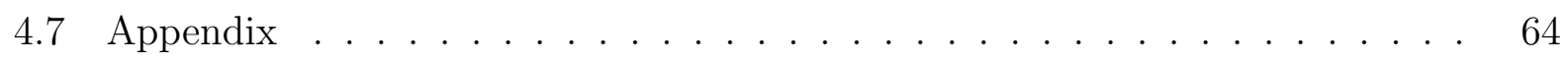

4.7 .1 Proof of Lemma $1 \ldots \ldots \ldots \ldots \ldots$

4.7 .2 Proof of Theorem $3 \ldots \ldots \ldots \ldots$ 
4.7 .3 Proof of Theorem $4 \ldots \ldots \ldots$

4.7 .4 Proof of Proposition $1 \ldots \ldots \ldots \ldots 71$

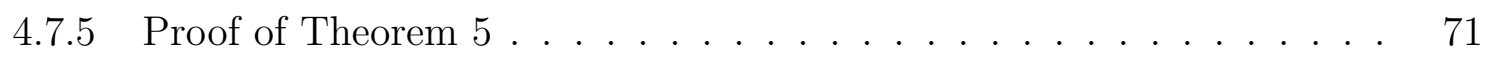

$\begin{array}{lll}5 & \text { Iterative Mechanisms for Electricity Markets } & \mathbf{7 4}\end{array}$

5.1 Introduction . . . . . . . . . . . . . . . . . . . . . 74

5.2 Preliminaries . . . . . . . . . . . . . . . . . . . . . . . . . . 79

5.2 .1 Constrained Economic Dispatch _ . . . . . . . . . . . . . 79

5.2 .2 Mechanism Design . . . . . . . . . . . . . . . . . . . . 79

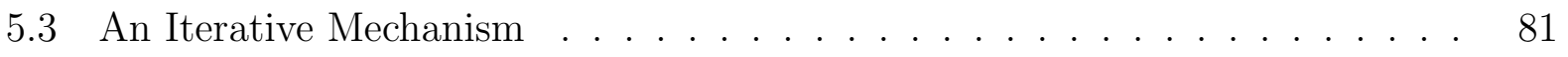

5.3 .1 Remarks . . . . . . . . . . . . . . . . . . . 83

5.4 Analysis . . . . . . . . . . . . . . . . . . . 84

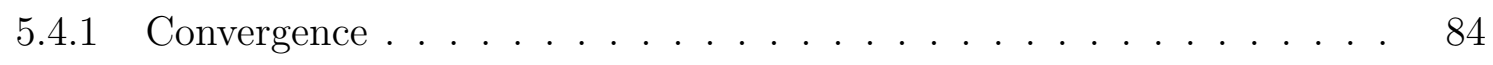

5.4 .2 Incentive Compatibility . . . . . . . . . . . . . . . 86

5.4.3 Participation Constraints and Budget Balance . . . . . . . . . . . 89

5.4 .4 Variations on a Theme . . . . . . . . . . . . . . . . . . 90

5.5 Iterative Mechanism for Stochastic Market Clearing . . . . . . . . . . . . 92

5.5 .1 Preliminaries . . . . . . . . . . . . . . . . . . . . . . 92

5.5 .2 Iterative Mechanism Design _ . . . . . . . . . . . . 95

5.5 .3 Analysis . . . . . . . . . . . . . . . . . . . . . . 97

5.6 Conclusions of this Chapter . . . . . . . . . . . . . . . . . . . . 99

5.7 Appendix A: Proof of Theorem $6 \ldots \ldots 101$

5.7 .1 Preliminary Results . . . . . . . . . . . . . . . . . . . . . . . 101

5.7 .2 Proof of Theorem $6 \ldots \ldots$. . . . . . . . . . . . . 103

5.8 Appendix B: Demand Aggregator and Generator Associated with Multiple Units . . . . . . . . . . . . . . . . . . . . . . . . . . . . . 104

$5.8 .1 \quad$ Mechanism Design . . . . . . . . . . . . . . . . . 105

5.8 .2 Analysis . . . . . . . . . . . . . . . . . . . 107

5.9 Appendix C: Proofs for Stochastic Market Clearing . . . . . . . . . . . 111 
5.9.1 Proof of Lemma 4. . . . . . . . . . . . . . 111

5.9.2 Proof of Theorem 9 . . . . . . . . . . . . . . 112

6 Conclusions $\quad 115$

6.1 Summary of the Dissertation . . . . . . . . . . . . . . . . . 115

6.2 Future Directions . . . . . . . . . . . . . . . . . . . . . . . 117

$\begin{array}{ll}\text { Bibliography } & 118\end{array}$ 


\section{LIST OF FIGURES}

3.1 Social foraging with and without mutual interactions with quadratic resource profile. . . . . . . . . . . . . . . . . . . . . . . 38

3.2 Social foraging with and without mutual interactions with non-quadratic re-

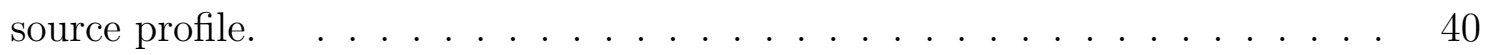

3.3 Social foraging within an incomplete graph with quadratic resource profile. 41

4.1 Performance comparison between the scheme based upon the average of $N=$ 10 gradient samples per step and the flocking-based approach with 10 fully connected threads. . . . . . . . . . . . . . . . . . 47

4.2 Performance comparison for Ackley's function (Case 1) . . . . . . . . . . . . 60

4.3 Performance comparison for Ackley's function (Case 2) . . . . . . . . . . . . 61 


\section{CHAPTER 1}

\section{Overview}

Many natural and engineering systems can be characterized as a collection of interacting agents each having access to local information, making local decisions, having local interactions with neighbors, and seeking to optimize local objectives. A representative sampling includes animal groups, social networks, autonomous vehicle teams, robot teams, sensor networks, and interconnected power systems. The analysis and design of such systems falls under the broader framework of "multi-agent control and optimization". Here depending on the context, "agents" could be animals, autonomous vehicles, power plants, etc. Multi-agent systems have the potential for solving problems which are difficult or impossible for an individual agent or monolithic system to solve. For instance, swarming behavior in nature is advantageous for avoiding predators and increasing the odds of finding food (see Grünbaum [38]). However, the complexity associated with a potentially large number of interacting agents brings about challenging control and optimization problems for system designers and coordinators. Such difficulty is often enhanced by the presence of noise/uncertainty that is pervasive in both biological and engineering systems.

In this dissertation, we focus on the analysis and design of several multi-agent control/optimization algorithms for various problems under uncertainty. Specifically, we first

develop a theory of noise reduction by swarming in the context of animal social foraging, which suggests that swarming-like approaches may provide an additional level of robustness for the control and/or optimization of networked agents. The theory is then applied to the problem of stochastic optimization, for which we propose a distributed swarming-like 
computing algorithmic scheme that relies on modest communication requirements amongst processors and most importantly, does not require synchronization. At last, we propose a class of iterative mechanisms to identify the optimal design in electricity markets under possibly noisy inputs from market participants. This class is shown to exhibit many desirable properties. In what follows, we present the background, motivation, and main results of each problem, respectively.

Swarming, flocking, schooling and other aggregations of organisms in groups has been studied extensively in biology (see $[64,65,74]$ for examples). There is empirical evidence that swarming is a useful strategy for social foraging in a noisy environment (see Grünbaum [38]). However, to our knowledge, there is no mathematical formalization of noise reduction properties of swarming in social foraging. In Chapter 3, we develop a mathematical model for analyzing the benefits of social foraging in a noisy environment. We identify conditions on the nutrient profile ensuring that local agent actions will lead to cohesive foraging. For convex, smooth nutrient profiles we formalize the way in which swarming for social foraging is better at handling the effects of noise when compared to the average of individual foraging strategies. Under a swarming discipline, observational noise realizations that induce trajectories differing too much from the group average are likely to be discarded because of each individual's need to maintain cohesion. As a result, the group trajectories are less affected by noise. Simulation experiments indicate our theoretical results are robust to inter-agent communication constraints and non-convex nutrient profiles. These results suggest that swarming-like approaches for the control and/or optimization of networked agents may offer an additional level of robustness.

In operations research, optimization is the selection of a best element (with regard to some criteria) from some set of available alternatives. In the optimization process, noise is present whenever physical system measurements or computer simulations are used for approximation. Examples include problems where data are collected while the system is operating, or problems where estimates are formed by computer-based Monte Carlo sampling according to a statistical distribution (see $[85,89]$ ). The performance of the single-thread 
stochastic gradient algorithm is highly sensitive to noise. Thus, there is a literature on estimation techniques leading to better gradient estimation often involving increasing sample size (see $[27,89]$ for a survey of gradient estimation techniques). When sampling is undertaken in parallel, synchronization is needed to execute the tasks that can not be executed in parallel. The speed-up obtained by parallel sampling and centralized gradient estimation is limited by overhead related to $(i)$ time spent gathering samples (which could be significant for example in the simulation of complex systems) and (ii) synchronization. When sampling times are not negligible and exhibit large variation, synchronization may cause significant overhead so that real-time performance of stochastic gradient algorithm based upon the average of $N$ samples obtained in parallel is highly affected by large sampling time variability.

Inspired by the aforementioned theory on noise reduction property of social foraging, in Chapter 4 we propose a distributed computing algorithmic scheme for stochastic optimization which relies on modest communication requirements amongst processors and most importantly, does not require synchronization. Specifically, we analyze a scheme with $N>1$ independent threads implementing each a stochastic gradient algorithm. The threads are coupled via a perturbation of the gradient (with attractive and repulsive forces) in a similar manner to mathematical models of flocking, swarming and other group formations found in nature with mild communication requirements (see Gazi and Passino [33]). When the objective function is convex, we show that a flocking-like approach for distributed stochastic optimization provides a noise reduction effect similar to that of a centralized stochastic gradient algorithm based upon the average of $N$ gradient samples at each step. The distributed nature of flocking makes it an appealing computational alternative. We show that when the overhead related to the time needed to gather $N$ samples and synchronization is not negligible, the flocking implementation outperforms a centralized stochastic gradient algorithm based upon the average of $N$ gradient samples at each step. When the objective function is not convex, the flocking-based approach seems better suited to escape locally optimal solutions due to the repulsive force which enforces a certain level of diversity in the set of candidate solutions. Here again, we show that the noise reduction effect is similar to 
that associated to the centralized stochastic gradient algorithm based upon the average of $N$ gradient samples at each step.

Electricity is a complex bundle of services involving multiple power plants and consumers in various locations over a capacity constrained network. A particular feature of many electricity markets is that market clearing is carried out by solving an optimization problem using input information (e.g., bids to buy and/or sell electricity) from market participants. The optimal design can be identified through a mechanism (see Silva et al. [87]), i.e., the rules by which market dispatch and payment to participants in an electricity market are determined while taking into account network and reliability constraints. In a direct mechanism, market participants (i.e., generators and retailers) report their private information (i.e., costs and willingness to pay) to the ISO or RTO which in turn clears the market and determines the associated market compensation and/or charges for each market participant. A mechanism is said to be incentive compatible in dominant strategies (or strategy-proof) if it is optimal for every market participant to truthfully report their private information regardless of the reporting strategies of other participants. A mechanism is said to be efficient if the market clears with the efficient market outcome. A weaker form of incentive compatibility is related to the implementation in a Bayes-Nash equilibrium. This concept relies on a common knowledge assumption on the market participants' private information (see Borgers et al. [8]). While direct mechanisms only require one round of interaction, the nature of the information exchange between market participants and the market-maker is quite complex. For example, in many designs currently in place, each generator (respectively, each retailer) must report their complete cost function (respectively, their willingness to pay) over the set of individual market outcomes. In this rich strategy space, equilibrium strategic behavior can be quite complex often inducing inefficient market outcomes (see for example, Anderson et al. [2] and Holmberg and Newbery [41]).

In Chapter 5, we propose a class of iterative mechanisms where market participants are asked to report the cost and willingness to pay for small adjustments at each iteration. A tentative market dispatch is marginally adjusted ensuring network constraints are satisfied, and 
a stopping criteria is checked. Small adjustments requiring minimal information from market participants at each iteration facilitate the identification of incentives for ensuring truthful reporting of private information. At each iteration, each market participant is charged (or compensated) according to the marginal change in surplus to all other market participants (see $[17,37]$ ). We show that truthful reporting is (approximately) a dominant strategy and that when the mechanism stops, the resulting economic dispatch is approximately efficient. Individual participation constraints are satisfied as the net surplus from trade is approximately shared equally by all participants and the mechanism is approximately weakly budget balanced.

The inherent intermittency of renewable technologies poses new challenges for ensuring a reliable and secure operation of electricity markets. As the marginal cost of renewable electricity is negligible, purely economic considerations would dictate a market clearing outcome in which most renewable output is cleared. However, intermittency may compromise the secure operation of the power grid infrastructure supporting the market. The incorporation of reliability and/or security constraints in market clearing further complicates the task of market design. In the second part of Chapter 5, we consider an iterative mechanism for stochastic market clearing (see $[10,78,107,113]$ ). This is an important feature as the share of renewable (intermittent) capacity in most electricity markets around the world is increasing at a steady pace and ex-post adjustments to market dispatch are the norm. In this case, the marginal cost of adjustments may only be estimated with some error. We show that truthful reporting is a Nash equilibrium and the resulting dispatch converges almost surely to the efficient dispatch.

\subsection{Main Contributions}

To summarize, we restate the main contributions of the dissertation as follows.

- We develop a mathematical model for analyzing the benefits of social foraging in a noisy environment, and identify conditions on the nutrient profile ensuring that local 
agent actions will lead to cohesive foraging.

- We formalize the way in which swarming for social foraging is better at handling the effects of noise when compared to the average of individual foraging strategies. These results suggest that swarming-like approaches for the control and/or optimization of networked agents may provide an additional level of robustness.

- We propose a distributed flocking-like computing algorithmic scheme for stochastic optimization which relies on modest communication requirements amongst processors and most importantly, does not require synchronization.

- We show that a flocking-like approach for distributed stochastic optimization provides a noise reduction effect similar to that of a centralized stochastic gradient algorithm based upon the average of $N$ gradient samples at each step. When the overhead related to the time needed to gather $N$ samples and synchronization is not negligible, the flocking implementation outperforms a centralized stochastic gradient algorithm based upon the average of $N$ gradient samples at each step.

- We propose a class of iterative mechanisms for electricity markets and show this class exhibits many desirable properties (approximately): incentive compatibility, efficiency, individual rationality and (weak) budget balance.

- We analyze an iterative mechanism for stochastic market clearing, in which the marginal cost of adjustments may only be estimated with some error. We show that truthful reporting is a Nash equilibrium and the resulting dispatch converges almost surely to the efficient dispatch.

\subsection{Organization of the Dissertation}

The rest of this dissertation is organized as follows. In Chapter 2, we introduce in more detail some background of the concepts used in this dissertation. In Chapter 3, we develop a mathematical model for analyzing the benefits of social foraging in a noisy environment and 
formalize the way in which swarming for social foraging is better at handling the effects of noise when compared to the average of individual foraging strategies. The results are then applied to the problem of stochastic optimization in Chapter 4 where we propose and analyze a distributed computing algorithmic scheme. In Chapter 5, we propose a class of iterative mechanisms to identify the optimal design in electricity markets under possibly noisy inputs from market participants. We conclude this dissertation in Chapter 6 with a discussion on future directions. 


\section{CHAPTER 2}

\section{Background}

In this chapter, we present some background of the concepts used in this dissertation in more detail. In particular, we review the literature on swarming in Section 2.1. In Section 2.2 we discuss some of the classical methods used in stochastic optimization. In Section 2.3 we present an introduction of electricity markets and the related mechanism design problem.

\subsection{Swarming}

In this section, we present a review of the literature on swarming. In biology, swarming is often referred to as the aggregation of organisms in groups. It can be found in many organisms ranging from simple bacteria to mammals. Examples of swarms include schools of fish, flocks of birds, herds of animals and colonies of bacteria (see Okubo [72]). Swarms have been studied extensively in biology (see $[11,19,22,25,64,65,67,68,72,92]$ ), physics (e.g., $[29,100,101])$ and engineering (e.g., [3,30,42,46,61,73,80, 94, 109]).

\subsubsection{Swarming in Biology and Physics}

Various models have been proposed to describe the swarming phenomena in biology and physics. Breder [11] is one of the earliest work to study swarming behavior mathematically. Inspired by earlier field work, the author proposed that within fish schools there exist attraction and repulsion forces depending on the distance between fish and fish. The repulsion is more effective at short distances, and the attraction dominates at greater distances. A 
simple model was given to represent such effects based on a "school" of two fishes. In the model, cohesiveness of a group is determined by the number of animals, distances between individuals or groups, and the attraction/repulsion forces. The author also discussed the masking or shadowing effects, that is, when the fish school is beyond a certain size, an individual cannot see all the other school members since some of them are "hidden" and out of sight. Therefore, the model is more suitable for small groups. Data from different species of fish were compared to validate the model.

In [72], Okubo introduced a random walk model and approximated it by an advectiondiffusion process. He then demonstrated a kinetic distinction between diffusion (random walk) and the motion of swarming or grouping individuals. By applying Newton's laws of motion, the author constructed ordinary differential equations to represent the dynamics of individual animals. In addition, various aspects of animal grouping were studied including group formation processes, grouping around multiple centers, and group size distributions. Finally, the presented mathematical models were compared with data of animal groupings such as insect swarms, zoo-plankton swarms, fish schools, bird flocks, and mammal herds.

In Vicsek et al. [100], a model with simple dynamics was introduced to investigate the emergence of self-ordered motion in systems of particles with biologically motivated interactions. In this model, particles are driven with a constant absolute velocity, which equals the average moving direction of the particles in their neighborhood along with some random perturbation. A continuous kinetic phase transition was observed in some of the numerical simulations. The observed behaviors were later theoretically explained by Jadbabaie et al. [46], who also derived convergence results for several other similarly inspired models. It was proved there that the Vicsek model is a graphic example of a switched stable linear system.

Swarming is often driven by the advantage of such collective and coordinated behavior in avoiding risk and foraging for food. Grünbaum presented evidence in [38] that schooling behavior can improve the ability of animals performing taxis to climb gradients, while asocial taxis would possibly be ineffective. In this paper, it was assumed that each swarming agent 
performs a biased random walk in a periodic domain, while its motion is affected by the behaviors and positions of its neighbors. The author argued that swarming facilitates taxis because the alignment tendency results in tight angular distributions within groups, thereby weakening the effects of individual sampling errors. As a result, more school members orient up-gradient than in the asocial case. This finding provided a mathematical reason why schooling and swarming is so frequently observed in nature.

Mogilner and Edelstein-Keshet [64] proposed continuum models for swarming behavior when non-local interactions exist. The model consists of integro-differential advectiondiffusion equations where convolution terms are used to describe long range attraction and repulsion. It was shown that the swarm of organisms can be long-lasting, though not globally stable. True locally stable solutions occur only when density dependence is included in the diffusion term. If the density dependence in the repulsion term is of a higher order than in the attraction term, then the swarm profile agrees with observations in biological examples, i.e., the swarm has a constant interior density with sharp edges.

Couzin et al. [19] introduced a self-organizing model of group formation in three dimensional space and used it to investigate the spatial dynamics of animal groups, e.g., fish schools and bird flocks. It was revealed that minor changes in individual-level interactions may result in major group-level behavioral transitions. The authors also presented the first evidence that "collective memory" exists in such animal groups during the transition of a group between distinct types of collective behaviors. The authors then used the model to show how individual differences influence group structure, and how individuals employing local rules accurately change their spatial position within a group.

Cucker and Smale [22] provided a model describing the evolution of a flock for both continuous and discrete time. In the model, every bird adjusts its velocity by adding to it a weighted average of the differences between its velocity and those of the other birds. A constant model parameter $\beta$ captures the rate of decay of the influence between birds in the flock. The authors gave conditions to ensure that the birds' velocities converge to a common one and the distances between birds remain bounded. In particular, when $\beta<1 / 2$ 
convergence of the flock to a common velocity is guaranteed, while for $\beta \geq 1 / 2$ convergence is ensured under some condition on the initial positions and velocities of the birds. Some of the follow-up studies on the model can be found in $[1,15,20,21,39]$.

Strömbom [92] proposed a self-propelled particle model in which attraction is the only force between individuals. The model was shown to generate three different phases: swarms, undirected mills and moving aligned groups. In the zero noise limit, these phases depend on the relative strength of attraction and individual inertia. By restricting the individuals' fields of vision and increasing the noise level in the system, the groups were found to generate both directed mills and three dynamically moving, "rotating chain" structures.

In Gazi [29], a Lagrangian dynamics based approach for modeling swarm dynamics was proposed. Different from the commonly used Newton dynamics that is based on forces, this approach is based on energy. The author introduced the concept of biological potential energy along with its various components, e.g., swarming potential energy, environmental potential energy and predator potential energy. Example kernel functions were discussed and numerical simulations were provided.

Finally, some general references on collective motion of organisms and mathematical models in biology include the review by Vicsek [101], the books by Parrish and Hamner [74], Edelshtein-Keshet [25], Murray [67,68], Britton [12] and Robeva [83].

\subsubsection{Swarming in Engineering}

Swarms have also been studied widely in the context of engineering applications, particularly in developing distributed cooperative control for autonomous multi-agent systems (see [3, $30,32,54,59,73,80,94])$.

Reynolds in his seminal work [80] explored a new approach to present the aggregate motion of a flock in computer animation. This approach was based on simulation, which is an alternative to scripting the paths of each bird individually. The simulated flock was regarded as an elaboration of a particle system with the simulated birds being the particles. The group motion of the simulated flock was realized utilizing a distributed behavioral model 
in which each bird choses its own course. Reynolds gave three rules to prescribe the flock behaviors, i.e., collision avoidance, velocity matching, and flock centering. The animations seem to correspond to an observer's intuition of "flock-like motion".

Significant swarm-related work has been done on formation control of multi-robot teams. In Balch [3], reactive behaviors for four formations and three formation reference types were presented. The desired behaviors were successfully demonstrated in both laboratory experiments and field tests. Suzuki and Yamashita [94] studied a multi-robot system where no global $x-y$ coordinate system exists. The authors investigated a number of formation problems of geometric patterns in the plane by robots. Algorithms were presented for converging and moving the robots to a single point in a finite number of steps. In addition, the paper characterized the class of geometric patterns that the robots can form given their initial configurations.

Vehicle groups may be regarded as another type of swarm. Leonard and Fiorelli [54] presented a framework for coordinated and distributed control of multiple autonomous vehicles using artificial potentials and virtual leaders. Artificial potentials inspired by biological models were designed to define interaction control forces between neighboring vehicles and enforce a desired inter-vehicle spacing. A virtual leader is a moving reference point that were used to manipulate group geometry and direct the motion of the group. The authors constructed a Lyapunov function to prove the stability of the system.

Early work on swarm stability include Gazi and Passino [30,32]. In [30] the authors specified an "individual-based" continuous-time model for swarm aggregation in $N$-dimensional space and studied its stability properties. It was shown that the individuals (autonomous agents or biological creatures) will form a cohesive swarm in a finite time. The authors also obtained an explicit bound on the swarm size, which depends only on the parameters of the swarm model. In [32], an additional factor determining the motion of each individual was introduced, that is, each individual is attracted to the more favorable regions and repulsed from the unfavorable regions. Hence the emergent behavior of the swarm motion is the result of a balance between inter-individual interactions and the environmental effects. Conditions 
for convergence to more favorable regions were provided.

Moreau [66] studied a model of network of agents interacting via time-dependent communication links. The model could be applied to various fields including synchronization, swarming and distributed decision making. In the model, agents update their states based on the information received from neighbors. The author provided necessary and/or sufficient conditions for the convergence of the individual agents' states to a common value, thus extending earlier results including those obtained in Jadbabaie et al. [46]. The stability analysis was done by using a blend of graph-theoretic and system-theoretic tools with the notion of convexity being crucial.

Olfati-Saber [73] presented a theoretical framework for design and analysis of distributed flocking algorithms. Three algorithms were proposed in both free space and in the presence of obstacles. It was shown that the first algorithm embodies all three rules of Reynolds [80], while leading to regular fragmentation. The second and third algorithm both lead to flocking. The author provided a systematic method for the construction of collective potentials for flocking. These potentials were used to penalize deviation from desired latticeshaped objects. Moreover, it was shown that migration of flocks can be preformed using a peer-to-peer network of agents.

Some more recent work on swarm stability and multi-agent control can be found in $[33,56-59,62,93,96,112]$. For a general review of multi-agent coordination and control we refer the readers to Cao et al. [14] and Bullo et al. [13].

Particle swarm optimization (PSO) $[18,86]$ is a stochastic population-based optimization technique motivated by the swarming behavior in nature. PSO searches a space by adjusting the trajectories of individual vectors that are called "particles". Each particle is defined within the context of a topological neighborhood, and its movement is influenced by its own best known position as well as its neighbors' best results. Shi [86] studied the effects of certain parameters on the performance of PSO. In Clerc and Kennedy [18], various properties of the algorithm were investigated and a generalized model was presented. For a review on the recent progress of PSO we refer readers to Bonyadi and Michalewicz [7]. 


\subsection{Stochastic Optimization}

In this section, we review some of the methods used in stochastic optimization (optimization in the presence of noisy observations).

Noise is present whenever physical system measurements or computer simulations are used for approximation. Examples include problems where data are collected while the system is operating, or problems where estimates are formed by computer-based Monte Carlo sampling according to a statistical distribution (see [85, 89]). The presence of noise might mislead an optimization algorithm throughout the entire process and result in false optimal solutions.

Sample average method is a widely accepted approximation approach in the presence of noisy observations (see $[27,52,85]$ ). The basic idea of such methods is that after a random sample is generated, the expected value is approximated by the corresponding sample average. The obtained sample average optimization problem is solved, and the procedure is repeated several times until a stopping criterion is satisfied. For instance, Kleywegt et al. [52] studied a Monte Carlo simulation-based approach to stochastic discrete optimization problems. It was shown that when the sample size increases, the optimal solution obtained from the sample average approximation problem provides the "exact" optimal solution with probability increasing at an exponential rate in the sample size $N$.

Stochastic approximation (SA) schemes have been popular for solving stochastic optimization problems. In 1951, Robbins and Monro in their seminal work [82] proposed a classical SA algorithm for solving a root finding problem, where the function $M(x)$ is represented as an expected value. It was assumed that there is a constant $\alpha$, such that the equation $M(x)=\alpha$ has a unique root at $x=\theta$. Though $M(x)$ cannot be directly observed, measurements of the random variable $N(x)$ can be obtained where $\mathbb{E}[N(x)]=M(x)$. The structure of the algorithm is to then generate iterates of the form: $x_{n+1}-x_{n}=a_{n}\left(\alpha-N\left(x_{n}\right)\right)$. Under suitable conditions, $x_{n}$ converges in $L^{2}$ (and hence also in probability) to $\theta$. A major insight of the algorithm was that, if the step-size sequence $a_{n}>0$ are chosen to satisfy $\sum_{n} a_{n}=\infty$ and 
$\sum_{n} a_{n}^{2}<\infty$, then there is an implicit averaging that eliminates the noise effects in the long run. Various modified SA algorithms have been proposed thereafter (see [35, 69, 77, 88, 111] for examples). These methods have been shown to be competitive with the sample average approach. For a comprehensive review of SA algorithms and applications we refer readers to $[9,53]$.

Recently there has been considerable interest in distributed implementation of stochastic gradient/subgradient algorithms (see for examples [16, 90, 98]). Srivastava and Nedić [90] considered the problem of reaching agreement on a set of local variables and the problem of minimizing the sum of local objective functions when the local variables are constrained to local convex sets. Two types of diminishing step size sequences were employed to account for communication noise and subgradient errors. In addition, asymptotic error bounds were established for the constant step size policy. Towfic and Sayed [98] studied distributed optimization over a network of learners in which each learner possesses a convex cost function. It was shown that when small constant step-sizes are employed, the expected distance between the optimal solution vector and that obtained at each node in the network can be made arbitrarily small. Dynamic convex optimization problems under noisy information exchange were investigated in Cavalcante and Stanczak [16]. It was assumed there that each agent has access to a local time-varying cost function, and the objective is to find a time-invariant minimizer of the sum of these local functions. The authors provided sufficient conditions for almost sure convergence of the algorithm as the number of iterations tend to infinity.

\subsection{Mechanism Design for Electricity Markets}

In this section, we present an introduction of electricity markets and the related mechanism design problem.

Electricity is a complex bundle of services involving multiple power plants and consumers in various locations over a capacity constrained network. A particular feature of many electricity markets is that market clearing is carried out by solving an optimization problem using input information (e.g., bids to buy and/or sell electricity) from market participants. 
This type of clearing is needed because other more traditional clearing protocols (e.g., bilateral trading) may not reliably or timely satisfy physical constraints. Market clearing is thus akin to a "black box" since the underlying optimization model is not necessarily transparent to market participants. The use of relatively sophisticated "black box" optimization models for clearing has important implications for market design. Some market participants because of their relative size, location or cost may have the ability to significantly alter market clearing outcomes by manipulating the information they provide to the ISO/RTO in charge of clearing. Depending upon the pricing rules in place, some market participants may find it advantageous to manipulate market clearing outcomes. This could possibly result in inefficient outcomes as well as distorted prices which provide incorrect investment incentives. Because of the "black box" nature of market clearing, it may be exceedingly hard to detect and/or identify certain types of market power abuse.

The history of electricity markets is fundamentally different from most commodity markets which gradually evolved towards standard terms of trade and institutions that helped increase liquidity and reduce transaction risks (see for example McMillan [63]). Most electricity markets evolved from the coordinated or pooled operation of vertically integrated (and highly regulated) utilities. This evolution was rather artificial in that it was imposed by governments intent on bringing an element of competition to an industry no longer considered a natural monopoly. Not surprisingly many alternative designs for the operation of electricity markets have been tried out by different countries. At times, concerns over economic efficiency have motivated comprehensive reforms. This was the case of the NETA reform in the UK (see $[26,48]$ ) according to which a uniform pricing rule was replaced by pay-as-bid pricing rule (among other important changes). More than thirty years after the first electricity market began operating, the task of properly tuning market rules to ensure economically efficient outcomes (i.e., market design) seems far from complete (see for example the discussion prompted by FERC's Notice of Proposed Rulemaking Docket No. RM16-5-000 regarding the relaxation of the price cap).

The identification of the optimal design for an electricity market can be formulated as a 
mechanism design problem (see Silva et al. [87]). Mechanism design is a field of game theory in which the rules of a game are designed in order to induce a desired equilibrium outcome resulting from the interaction of rational agents with private information (see Borgers et al. [8]). In a direct mechanism, market participants (i.e., generators and retailers) report their private information (i.e., costs and willingness to pay) to the ISO or RTO which in turn clears the market and determines the associated market compensation and/or charges for each market participant. A mechanism is said to be incentive compatible in dominant strategies (or strategy-proof) if it is optimal for every market participant to truthfully report their private information regardless of the reporting strategies of other participants. A mechanism is said to be efficient if the market clears with the efficient market outcome. A weaker form of incentive compatibility is related to implementation in a Bayes-Nash equilibrium. This concept relies on a common knowledge assumption on the market participants' private information (see Borgers et al. [8]). 


\section{CHAPTER 3}

\section{Noise Reduction by Swarming in Social Foraging}

In this chapter, we develop a mathematical model for analyzing the benefits of social foraging in a noisy environment. We identify conditions on the nutrient profile ensuring that local agent actions will lead to cohesive foraging. For convex, smooth nutrient profiles we formalize the way in which swarming for social foraging is better at handling the effects of noise when compared to the average of individual foraging strategies. Under a swarming discipline, observational noise realizations that induce trajectories differing too much from the group average are likely to be discarded because of each individual's need to maintain cohesion. As a result, the group trajectories are less affected by noise. Simulation experiments indicate that our theoretical results are robust to inter-agent communication constraints and nonconvex nutrient profiles. These results suggest that swarming-like approaches for the control of networked agents may provide an additional level of robustness.

\subsection{Introduction}

Swarming, flocking, schooling and other aggregations of organisms in groups have been studied extensively in biology (see $[64,65,74]$ ). Organisms in swarms can exploit several advantages of staying close to each other for more effective foraging. For example, in [38] Grünbaum explained how social foragers more successfully perform chemotaxis over noisy gradients than individuals. Such biological advantage was also demonstrated in Passino [76] by modeling the behavior of E. coli and M. xanthus bacteria. 
There exists a growing literature on mathematical models for swarming behavior aimed at formalizing the delicate balance between inter-individual interactions and the individual interaction with the environment. For example, in $[32,59]$ the authors developed a model in which the motion of each individual is affected by an attraction potential to the other individuals (over long distances), a repulsion potential from the other individuals (on short distances) and lastly, attraction to locations with more favorable nutrient profiles. Swarms have also been studied in physics (see $[97,100]$ ) where the collective behavior of "self-propelled particles" is studied, and in the context of engineering applications for developing distributed cooperative control, coordination, and learning strategies for autonomous multi-agent systems (see $[42,61,94,108,109]$ for examples).

Sensing or observational noise/error makes foraging a more difficult task (see $[102,103])$. Liu and Passino [59] investigated a model on foraging in a noisy environment, but the observation noise was assumed to be bounded, and only a specific nutrient profile (i.e., a "plain" resource profile) was considered. The advantage of social foraging over individual action was not formalized either. Noise effects have also been considered in the context of bio-inspired extremum seeking (see $[91,109,114]$ ). In Stanković and Stipanović [91], a stochastic-approximation type protocol was proposed to attenuate noise effects, while Zou et al. [114] adopted a modified PSO (particle swarm optimization) method. Wu and Zhang [109] analyzed a bio-inspired scheme for robotic sensing agents which was shown to reduce noise. Agents share all measurements so that an $H_{\infty}$ filter can be implemented. They switch from cooperative exploration to individual exploration when a significantly improved signal-to-noise ratio (SNR) is obtained. In our setting to be described below, agents do not attempt to explicitly cooperate in order to filter out noise. Instead, each agent moves in a response to independent noisy measurements of the underlying nutrient potential which is further perturbed by a swarming (or flocking) potential (a function of relative coordinates of neighboring agents).

Collective behaviors other than swarming may also reduce noise in a system. For example, Tabareau et al. [95] explains mathematically why synchronization may help protect 
interconnected nonlinear dynamic systems from noise. Specifically, synchronization makes the state trajectories of the noisy system approach that of a noise-free one. Nevertheless, swarming is distinct from synchronization in that swarm agents tend to keep a distance from each other. Moreover, the group objective of foraging is fundamentally different from that of synchronizing an interconnected nonlinear dynamic system.

There is empirical evidence that swarming is a useful strategy for social foraging in a noisy environment (see Grünbaum [38]). However, to our knowledge, there is no mathematical formalization of noise reduction properties of swarming in social foraging. In this chapter we develop a mathematical model for analyzing the benefits of social foraging in a noisy environment. We identify conditions on the nutrient profile ensuring that local agent actions will lead to cohesive foraging. For convex, smooth nutrient profiles we formalize the way in which swarming for social foraging is better at handling the effects of noise when compared to the average of individual foraging strategies. Under a swarming discipline, observational noise realizations that induce trajectories differing too much from the group average are likely to be discarded because of each individual's need to maintain cohesion. As a result, the group trajectories are less affected by noise. We report results of a simulation testbed in which many of our standing assumptions are relaxed. The simulation experiments indicate that our theoretical results are robust to inter-agent communication constraints and non-convex nutrient profiles.

The remainder of this chapter is organized as follows. In Section 3.2 some preliminaries are provided. Section 3.3 introduces the model for agents, mutual interactions, and the foraging environment. In Section 3.4, swarm cohesion analysis is performed under conditions satisfied by a wide class of nutrient profiles. We analyze social foraging properties within the swarm in Section 3.5 assuming that the nutrient profile is strongly convex. Simulation examples are provided in Section 3.6. We conclude this chapter in Section 3.7. 


\subsection{Preliminaries}

In the analysis, we shall make use of certain concepts in graph theory and Brownian motion which we briefly review below.

A graph $G$ is a pair $(\mathcal{V}, \mathcal{E})$, where $\mathcal{V}$ is a set of vertices and $\mathcal{E}$ is a subset of $\mathcal{V} \times \mathcal{V}$ called edges (see Godsil and Royle [36]). The adjacency matrix $\left[\alpha_{i j}\right] \in \mathbb{R}$ of a graph is a matrix with nonzero elements satisfying the property $\alpha_{i j}>0 \Leftrightarrow(i, j) \in \mathcal{E}$. Self-joining edges are excluded, i.e., $\alpha_{i i}=0, \forall i$.

A one-dimensional Brownian motion is a real-valued stochastic process $B(t), t \geq 0$ that has the following properties (see Durrett [23]):

(i) If $t_{0}<t_{1}<\cdots<t_{n}$, then $B\left(t_{0}\right), B\left(t_{1}\right)-B\left(t_{0}\right), \ldots B\left(t_{n}\right)-B\left(t_{n-1}\right)$ are independent.

(ii) If $s, t>0$, then

$$
\mathbb{P}(B(s+t)-B(s) \in A)=\int_{A}(2 \pi t)^{-1 / 2} \exp \left(-x^{2} / 2 t\right) d x
$$

where $A \subset \mathbb{R}$ and $\mathbb{P}(\cdot)$ denotes the probability function.

(iii) With probability $1, t \rightarrow B(t)$ is continuous.

Notice that (ii) indicates that $B(s+t)-B(s)$ has a normal distribution with mean 0 and variance $t$.

The Kronecker product of matrices $A \in \mathbb{R}^{n \times n}$ and $B \in \mathbb{R}^{l \times l}$ is an $n l \times n l$ block matrix defined as follows:

$$
A \otimes B=\left(\begin{array}{cccc}
a_{11} B & a_{12} B & \ldots & a_{1 n} B \\
a_{21} B & a_{22} B & \ldots & a_{2 n} B \\
\vdots & \vdots & \ddots & \ldots \\
a_{n 1} B & a_{n 2} B & \ldots & a_{n n} B
\end{array}\right)
$$

$|\cdot|$ denotes the absolute value function. 


\subsection{The Swarm Model}

We adopt a similar swarm model as in Gazi and Passino [32] and consider a swarm composed of an interconnection of $N$ "agents" moving in an $m$-dimensional space. The equation of motion we consider for agent $i$ is given by

$$
d x_{i}=u_{i} d t
$$

where $x_{i} \in \mathbb{R}^{m}$ is the state of agent $i$ and $u_{i} \in \mathbb{R}^{m}$ is its control input. This is a kinetic model since it ignores the lower-level dynamics of the individual agents. Nevertheless, a kinetic model can be viewed as an approximation of a model with point mass swarm member dynamics for some organisms such as bacteria (see Gazi and Passino [32]).

We consider a control input given by

$$
u_{i}=\sum_{j=1, j \neq i}^{N} \alpha_{i j} g\left(x_{i}-x_{j}\right)-\nabla \sigma\left(x_{i}\right)+\sqrt{2 T} \frac{d B_{i}}{d t},
$$

where $g(\cdot)$ represents the function of mutual attraction and repulsion between the individuals and is an odd function of the form (see Gazi and Passino [31])

$$
g(y)=-y\left[g_{a}(\|y\|)-g_{r}(\|y\|)\right]
$$

where $g_{a}: \mathbb{R}^{+} \rightarrow \mathbb{R}^{+}$represents (the magnitude of) the attraction term and it has long range, whereas $g_{r}: \mathbb{R}^{+} \rightarrow \mathbb{R}^{+}$represents (the magnitude of) the repulsion term and it has short range, and $\|\cdot\|$ is the Euclidean norm. Assume that there exists an equilibrium distance $\rho>0$ such that $g_{a}(\rho)=g_{r}(\rho)$. For $\|y\|>\rho$ we have $g_{a}(\|y\|)>g_{r}(\|y\|)$, and for $\|y\|<\rho$ we have $g_{a}(\|y\|)<g_{r}(\|y\|)$. In this work we will consider linear attraction functions, i.e., $g_{a}(\|y\|)=a$ for some $a>0$ and all $\|y\|$, and repulsion functions satisfying

$$
g_{r}(\|y\|)\|y\|^{2} \leq b
$$

uniformly for some $b>0$.

The term $-\nabla \sigma\left(x_{i}\right)$ represents the motion of the individuals toward regions with higher nutrient concentration and away from regions with high concentration of toxic substances. 
Note that the implicit assumption that the individuals know the gradient of the profile (with noise/sensing errors) at their positions is not very restrictive since it is known that some organisms such as bacteria are able to construct local approximations to gradients (see Passino [76] for instance). In particular, $\sigma(x)$ is assumed to be differentiable everywhere and attains its minimum at $x=\mathbf{0}$, i.e., the nutrient concentration peaks at the origin.

$A=\left[\alpha_{i j}\right] \in \mathbb{R}^{N \times N}$ denotes the coupling matrix with $\alpha_{i j} \in\{0,1\} . \alpha_{i j}=1$ indicates that agent $i$ interacts with agent $j$. We assume that the corresponding graph $\mathcal{G}$ is undirected $\left(A=A^{T}\right)$ and connected, that is, there exists a path between any two different vertices in $\mathcal{V}$.

Unlike the model studied in $[32,59]$, we are assuming that the measurement of gradient is subject to white noise $\sqrt{2 T} d B_{i} / d t$ of uniform strength $\sqrt{2 T}$, and $B_{i}$ is an $m$-dimensional Brownian motion. This noise term is essentially the continuous-time counterpart of Gaussian white noise sequence in discrete time (see Cucker and Mordecki [21], Section 4.1 for reference). It follows that $\left\{x_{i}(t) \mid t \geq 0\right\}$ is a stochastic process governed by the stochastic differential equation (3.4). Notice that white noise is widely used in the literature (see $[21,39,95])$. Its structure allows us to quantify the effects of swarming on the social foraging process.

In the following sections, we will first perform cohesion analysis for the swarm under conditions satisfied by a wide class of nutrient profiles. Then we will study social foraging properties of the swarm under noise and compare them with the average performance of individuals.

\subsection{Swarm Cohesion Analysis}

\subsubsection{Preliminaries: Macro-micro Decomposition}

To facilitate analysis, we introduce a "macro-micro" decomposition which disintegrate the system into two parts: one system that describes the macroscopic dynamics and a second system that describes the microscopic dynamics. We start by setting the macroscopic part 
for the solution as the system's center of mass:

$$
\bar{x}=\frac{1}{N} \sum_{i=1}^{N} x_{i}
$$

The microscopic variables are then taken as the fluctuations with respect to the center of mass:

$$
e_{i}=x_{i}-\bar{x}
$$

Recall that

$$
d x_{i}=u_{i} d t=\sum_{j=1, j \neq i}^{N} \alpha_{i j} g\left(x_{i}-x_{j}\right) d t-\nabla \sigma\left(x_{i}\right) d t+\sqrt{2 T} d B_{i}
$$

where $g(\cdot)$ is an odd function. The macroscopic and microscopic parts of (3.4) can be written as

$$
\begin{aligned}
d \bar{x} & =\frac{1}{N} \sum_{i=1}^{N}\left[\sum_{j=1, j \neq i}^{N} \alpha_{i j} g\left(x_{i}-x_{j}\right) d t-\nabla \sigma\left(x_{i}\right) d t+\sqrt{2 T} d B_{i}\right] \\
& =-\frac{1}{N} \sum_{i=1}^{N} \nabla \sigma\left(x_{i}\right) d t+\frac{\sqrt{2 T}}{N} \sum_{i=1}^{N} d B_{i}
\end{aligned}
$$

and

$$
\begin{aligned}
d e_{i}=\sum_{j=1, j \neq i}^{N} \alpha_{i j} g\left(x_{i}-x_{j}\right) d t-\nabla \sigma\left(x_{i}\right) d t+\frac{1}{N} \sum_{i=1}^{N} \nabla \sigma\left(x_{i}\right) d t & \\
& +\sqrt{2 T} d B_{i}-\frac{\sqrt{2 T}}{N} \sum_{i=1}^{N} d B_{i}
\end{aligned}
$$

respectively. In light of (3.1),

$$
g\left(x_{i}-x_{j}\right)=-\left(x_{i}-x_{j}\right)\left[g_{a}\left(\left\|x_{i}-x_{j}\right\|\right)-g_{r}\left(\left\|x_{i}-x_{j}\right\|\right)\right]=-\left(x_{i}-x_{j}\right)\left[a-g_{r}\left(\left\|x_{i}-x_{j}\right\|\right)\right] .
$$

We have

$$
\sum_{j=1, j \neq i}^{N} \alpha_{i j} g\left(x_{i}-x_{j}\right)=-a \sum_{j=1, j \neq i}^{N} \alpha_{i j}\left(x_{i}-x_{j}\right)+\sum_{j=1, j \neq i}^{N} \alpha_{i j} g_{r}\left(\left\|x_{i}-x_{j}\right\|\right)\left(x_{i}-x_{j}\right) .
$$


Consequently, (3.6) becomes

$$
\begin{array}{r}
d e_{i}=-a \sum_{j=1, j \neq i}^{N} \alpha_{i j}\left(x_{i}-x_{j}\right) d t+\sum_{j=1, j \neq i}^{N} \alpha_{i j} g_{r}\left(\left\|x_{i}-x_{j}\right\|\right)\left(x_{i}-x_{j}\right) d t-\nabla \sigma\left(x_{i}\right) d t \\
+\frac{1}{N} \sum_{j=1}^{N} \nabla \sigma\left(x_{j}\right)+\sqrt{2 T} d B_{i}-\frac{\sqrt{2 T}}{N} \sum_{j=1}^{N} d B_{j} .
\end{array}
$$

\subsubsection{Swarm Cohesion}

We begin our study of the swarm behavior by investigating the cohesiveness of the swarm under some general conditions. To this end, let

$$
V_{i}=(1 / 2)\left\|e_{i}\right\|^{2}
$$

and define a Lyapunov function

$$
\bar{V}=(1 / N) \sum_{i=1}^{N} V_{i}
$$

to indicate the group's cohesiveness. We then define asymptotic swarming for the stochastic system (3.4):

Definition 1. (Swarming) System (3.4) has a (time-asymptotic) swarming if and only if the ensemble average of the Lyapunov function $\bar{V}$ is uniformly bounded in time $t$.

Applying Ito's formula ${ }^{1}$ to $V_{i}=(1 / 2)\left\|e_{i}\right\|^{2}$,

$$
\begin{aligned}
d V_{i}= & d e_{i} \cdot e_{i}+\frac{1}{2} d e_{i} \cdot d e_{i} \\
= & -a \sum_{j=1, j \neq i}^{N} \alpha_{i j}\left(x_{i}-x_{j}\right)^{T} e_{i} d t+\sum_{j=1, j \neq i}^{N} \alpha_{i j} g_{r}\left(\left\|x_{i}-x_{j}\right\|\right)\left(x_{i}-x_{j}\right)^{T} e_{i} d t \\
& -\left[\nabla \sigma\left(x_{i}\right)-\frac{1}{N} \sum_{j=1}^{N} \nabla \sigma\left(x_{j}\right)\right]^{T} e_{i} d t+\left[\sqrt{2 T} d B_{i}-\frac{\sqrt{2 T}}{N} \sum_{j=1}^{N} d B_{j}\right]^{T} e_{i} \\
& +m T\left(1-\frac{1}{N}\right) d t
\end{aligned}
$$

\footnotetext{
${ }^{1}$ See $\varnothing k s e n d a l[71]$ for a reference on stochastic differential equations.
} 
It follows that

$$
\begin{aligned}
d \bar{V}= & \frac{1}{N} d V_{i} \\
= & -\frac{a}{N} \sum_{i=1}^{N} \sum_{j=1, j \neq i}^{N} \alpha_{i j}\left(x_{i}-x_{j}\right)^{T} e_{i} d t+\frac{1}{N} \sum_{i=1}^{N} \sum_{j=1, j \neq i}^{N} \alpha_{i j} g_{r}\left(\left\|x_{i}-x_{j}\right\|\right)\left(x_{i}-x_{j}\right)^{T} e_{i} d t \\
& -\frac{1}{N} \sum_{i=1}^{N} \nabla^{T} \sigma\left(x_{i}\right) e_{i} d t+\frac{\sqrt{2 T}}{N} \sum_{i=1}^{N} d B_{i}^{T} e_{i}+m T \frac{(N-1)}{N} d t \\
= & -\frac{a}{N} \sum_{i=1}^{N} \sum_{j=1, j \neq i}^{N} \alpha_{i j}\left(x_{i}-x_{j}\right)^{T} e_{i} d t+\frac{1}{2 N} \sum_{i=1}^{N} \sum_{j=1, j \neq i}^{N} \alpha_{i j} g_{r}\left(\left\|x_{i}-x_{j}\right\|\right)\left\|x_{i}-x_{j}\right\|^{2} d t \\
& -\frac{1}{N} \sum_{i=1}^{N} \nabla^{T} \sigma\left(x_{i}\right) e_{i} d t+\frac{\sqrt{2 T}}{N} \sum_{i=1}^{N} d B_{i}^{T} e_{i}+m T \frac{(N-1)}{N} d t .
\end{aligned}
$$

Let $L=\left[l_{i j}\right]$ be the Laplacian matrix associated with the adjacency matrix $A$, with $l_{i i}=\sum_{j} \alpha_{i j}$ and $l_{i j}=-\alpha_{i j}$ when $i \neq j$. Denote by $\operatorname{Tr}(L)$ the trace of $L$. For an undirected graph, the Laplacian matrix is symmetric positive semi-definite (see Godsil and Royle [36] for reference). Notice that

$$
-\sum_{i=1}^{N} \sum_{j=1, j \neq i}^{N} \alpha_{i j}\left(e_{i}-e_{j}\right)^{T} e_{i}=\sum_{i=1}^{N} \sum_{j=1}^{N} l_{i j}\left(e_{i}-e_{j}\right)^{T} e_{i}=\sum_{i=1}^{N} \sum_{j=1}^{N} l_{i j} e_{j}^{T} e_{i} .
$$

Let $\mathbf{e}=\left[e_{1}^{T}, \ldots, e_{N}^{T}\right]^{T}$. Since graph $\mathcal{G}$ is connected, $\lambda_{2}(L)>0$. We have (refer to Godsil and Royle [36])

$$
\sum_{i=1}^{N} \sum_{j=1}^{N} l_{i j} e_{j}^{T} e_{i}=\mathbf{e}^{T}\left(L \otimes I_{m}\right) \mathbf{e} \geq \lambda_{2} \mathbf{e}^{T} \mathbf{e}=\lambda_{2} \sum_{i=1}^{N}\left\|e_{i}\right\|^{2},
$$

where $\lambda_{2}:=\lambda_{2}(L)$ is the second-smallest eigenvalue of $L$. It is also called the algebraic connectivity of $\mathcal{G}$.

In conclusion,

$$
\begin{aligned}
d \bar{V} \leq-\frac{a \lambda_{2}}{N} \sum_{i=1}^{N}\left\|e_{i}\right\|^{2} d t & +\frac{1}{2 N} \sum_{i=1}^{N} \sum_{j=1, j \neq i}^{N} \alpha_{i j} g_{r}\left(\left\|x_{i}-x_{j}\right\|\right)\left\|x_{i}-x_{j}\right\|^{2} d t \\
& -\frac{1}{N} \sum_{i=1}^{N} \nabla^{T} \sigma\left(x_{i}\right) e_{i} d t+\frac{\sqrt{2 T}}{N} \sum_{i=1}^{N} d B_{i}^{T} e_{i}+m T \frac{(N-1)}{N} d t .
\end{aligned}
$$

We now make the following standing assumptions. 
Assumption 1. (Strong convexity) The gradient of the resource profile satisfies

$$
(\nabla \sigma(y)-\nabla \sigma(x))^{T}(y-x) \geq \kappa\|y-x\|^{2}
$$

for some $\kappa>0$ and for all $x, y$.

Assumption 2. (Bounded gradient) There exists $\eta>0$ such that $\|\nabla \sigma(y)\| \leq \eta$, for all $y$.

Assumption 2 requires only that the gradient of the profile is bounded. This is a fairly mild assumption which is satisfied by most nutrient profiles used in biological models. The following result provides a characterization of the swarm cohesiveness.

Theorem 1. Suppose that either Assumption 1 or Assumption 2 hold. Then, the ensemble average of $\bar{V}$ is uniformly bounded in $t$.

1. If Assumption 1 is satisfied, then

$$
\mathbb{E}[\bar{V}] \leq e^{-2\left(\kappa+a \lambda_{2}\right) t} \bar{V}(0)+\left[\frac{b|\operatorname{Tr}(L)|}{4 N\left(\kappa+a \lambda_{2}\right)}+\frac{m T(N-1)}{2 N\left(\kappa+a \lambda_{2}\right)}\right]\left[1-e^{-2\left(\kappa+a \lambda_{2}\right) t}\right] .
$$

In the long run,

$$
\mathbb{E}[\bar{V}] \leq \psi_{1}^{*}=\frac{b|\operatorname{Tr}(L)|}{4 N\left(\kappa+a \lambda_{2}\right)}+\frac{m T(N-1)}{2 N\left(\kappa+a \lambda_{2}\right)} .
$$

2. If Assumption 2 is satisfied, then

$$
\mathbb{E}[\bar{V}] \leq e^{-c_{1} t} \bar{V}(0)+\frac{c_{2}}{c_{1}}\left(1-e^{-c_{1} t}\right) .
$$

where

$$
c_{1} \in\left(0,2 a \lambda_{2}\right) \text { is arbitrary, and } c_{2}=\frac{\eta^{2}}{2\left(2 a \lambda_{2}-c_{1}\right)}+\frac{b|\operatorname{Tr}(L)|}{2 N}+m T \frac{(N-1)}{N} .
$$

In particular,

$$
\mathbb{E}[\bar{V}] \leq \max \left\{\bar{V}(0), \psi_{2}^{*}\right\}
$$

and in the long run, $\mathbb{E}[\bar{V}] \leq \psi_{2}^{*}$. Here

$$
\psi_{2}^{*}=\frac{1}{2 a \lambda_{2}}\left[\sqrt{\frac{\eta^{2}}{4 a \lambda_{2}}+\frac{b|\operatorname{Tr}(L)|}{2 N}+m T \frac{(N-1)}{N}}+\sqrt{\frac{\eta^{2}}{4 a \lambda_{2}}}\right]^{2} .
$$


Proof. Case 1: Refer to Equation (3.11). Notice that

$$
\sum_{i=1}^{N} \nabla^{T} \sigma\left(x_{i}\right) e_{i}=\sum_{i=1}^{N}\left(\nabla^{T} \sigma\left(x_{i}\right)-\nabla^{T} \sigma(\bar{x})\right)\left(x_{i}-\bar{x}\right) \geq \sum_{i=1}^{N} \kappa\left\|x_{i}-\bar{x}\right\|^{2}=2 \kappa N \bar{V}
$$

where the inequality follows from Assumption 1. By (3.2),

$$
g_{r}\left(\left\|x_{i}-x_{j}\right\|\right)\left\|x_{i}-x_{j}\right\|^{2} \leq b
$$

It follows that

$$
\begin{aligned}
d \bar{V} \leq & -\frac{a \lambda_{2}}{N} \sum_{i=1}^{N}\left\|e_{i}\right\|^{2} d t+\frac{1}{2 N} \sum_{i=1}^{N} \sum_{j=1, j \neq i}^{N} \alpha_{i j} g_{r}\left(\left\|x_{i}-x_{j}\right\|\right)\left\|x_{i}-x_{j}\right\|^{2} d t \\
& -\frac{1}{N} \sum_{i=1}^{N} \nabla^{T} \sigma\left(x_{i}\right) e_{i} d t+\frac{\sqrt{2 T}}{N} \sum_{i=1}^{N} d B_{i}^{T} e_{i}+m T \frac{(N-1)}{N} d t \\
\leq & -2 a \lambda_{2} \bar{V} d t-2 \kappa \bar{V} d t+\frac{b|T r(L)|}{2 N} d t+m T \frac{(N-1)}{N} d t+\frac{\sqrt{2 T}}{N} \sum_{i=1}^{N} d B_{i}^{T} e_{i} .
\end{aligned}
$$

We then have

$$
\begin{aligned}
d\left[e^{2\left(\kappa+a \lambda_{2}\right) t} \bar{V}\right] & =e^{2\left(\kappa+a \lambda_{2}\right) t} d \bar{V}+2\left(\kappa+a \lambda_{2}\right) e^{2\left(\kappa+a \lambda_{2}\right) t} \bar{V} d t \\
& \leq\left[\frac{b|\operatorname{Tr}(L)|}{2 N}+m T \frac{(N-1)}{N}\right] e^{2\left(\kappa+a \lambda_{2}\right) t} d t+\frac{\sqrt{2 T}}{N} e^{2\left(\kappa+a \lambda_{2}\right) t} \sum_{i=1}^{N} d B_{i}^{T} e_{i} .
\end{aligned}
$$

Integrating both sides,

$$
\begin{aligned}
\bar{V} \leq e^{-2\left(\kappa+a \lambda_{2}\right) t} \bar{V}(0)+\left[\frac{b|\operatorname{Tr}(L)|}{4 N\left(\kappa+a \lambda_{2}\right)}+\frac{m T(N-1)}{2 N\left(\kappa+a \lambda_{2}\right)}\right]\left[1-e^{-2\left(\kappa+a \lambda_{2}\right) t}\right] \\
+e^{-2\left(\kappa+a \lambda_{2}\right) t} \int_{0}^{t} \frac{\sqrt{2 T}}{N} e^{2\left(\kappa+a \lambda_{2}\right) s} \sum_{i=1}^{N} d B_{i}^{T}(s) e_{i} .
\end{aligned}
$$

Taking ensemble average yields

$$
\mathbb{E}[\bar{V}] \leq e^{-2\left(\kappa+a \lambda_{2}\right) t} \bar{V}(0)+\left[\frac{b|\operatorname{Tr}(L)|}{4 N\left(\kappa+a \lambda_{2}\right)}+\frac{m T(N-1)}{2 N\left(\kappa+a \lambda_{2}\right)}\right]\left[1-e^{-2\left(\kappa+a \lambda_{2}\right) t}\right] .
$$

In the long run,

$$
\mathbb{E}[\bar{V}] \leq \psi_{1}^{*}=\frac{b|\operatorname{Tr}(L)|}{4 N\left(\kappa+a \lambda_{2}\right)}+\frac{m T(N-1)}{2 N\left(\kappa+a \lambda_{2}\right)}
$$


Case 2: Assumption 2 holds. By Equation (3.11) and (3.2),

$$
\begin{aligned}
d \bar{V} \leq & -2 a \lambda_{2} \bar{V} d t+\frac{b|\operatorname{Tr}(L)|}{2 N} d t+m T \frac{(N-1)}{N} d t+\frac{1}{N} \sum_{i=1}^{N} \eta\left\|e_{i}\right\| d t+\frac{\sqrt{2 T}}{N} \sum_{i=1}^{N} d B_{i}^{T} e_{i} \\
\leq & -2 a \lambda_{2} \bar{V}_{t} d t+\left[\frac{b|\operatorname{Tr}(L)|}{2 N}+m T \frac{(N-1)}{N}\right] d t+\sqrt{2} \eta \sqrt{\bar{V}_{t}} d t+\frac{\sqrt{2 T}}{N} \sum_{i=1}^{N} d B_{i}^{T} e_{i} \\
= & -c_{1} \bar{V} d t-\left(2 a \lambda_{2}-c_{1}\right) \bar{V} d t+\sqrt{2} \eta \sqrt{\bar{V}} d t+\left[\frac{b|\operatorname{Tr}(L)|}{2 N}+m T \frac{(N-1)}{N}\right] d t \\
& +\frac{\sqrt{2 T}}{N} \sum_{i=1}^{N} d B_{i}^{T} e_{i} \\
= & -c_{1} \bar{V} d t-\left(2 a \lambda_{2}-c_{1}\right)\left[\sqrt{\bar{V}}-\frac{\sqrt{2} \eta}{2\left(2 a \lambda_{2}-c_{1}\right)}\right]^{2} \\
& +\left[\frac{\eta^{2}}{2\left(2 a \lambda_{2}-c_{1}\right)}+\frac{b|T r(L)|}{2 N}+m T \frac{(N-1)}{N}\right] d t+\frac{\sqrt{2 T}}{N} \sum_{i=1}^{N} d B_{i}^{T} e_{i} \\
\leq & -c_{1} \bar{V} d t+c_{2} d t+\frac{\sqrt{2 T}}{N} \sum_{i=1}^{N} d B_{i}^{T} e_{i},
\end{aligned}
$$

where

$$
c_{1} \in\left(0,2 a \lambda_{2}\right) \text { is arbitrary, } c_{2}=\frac{\eta^{2}}{2\left(2 a \lambda_{2}-c_{1}\right)}+\frac{b|\operatorname{Tr}(L)|}{2 N}+m T \frac{(N-1)}{N} \text {. }
$$

Applying Ito's formula to $e^{c_{1} t} \bar{V}$,

$$
d\left(e^{c_{1} t} \bar{V}\right)=e^{c_{1} t} d \bar{V}+c_{1} e^{c_{1} t} \bar{V} d t \leq c_{2} e^{c_{1} t} d t+\frac{\sqrt{2 T}}{N} e^{c_{1} t} \sum_{i=1}^{N} d B_{i}^{T} e_{i} .
$$

Integrating the stochastic differential inequality,

$$
\bar{V}_{t} \leq e^{-c_{1} t} \bar{V}(0)+\frac{c_{2}}{c_{1}}\left(1-e^{-c_{1} t}\right)+e^{-c_{1} t} \int_{0}^{t} \frac{\tau}{N} e^{c_{1} s} \sum_{i=1}^{N} d B_{i}^{T} e_{i}
$$

Taking an ensemble average on both sides yields

$$
\mathbb{E}[\bar{V}] \leq e^{-c_{1} t} \bar{V}(0)+\frac{c_{2}}{c_{1}}\left(1-e^{-c_{1} t}\right)
$$


It follows that

$$
\mathbb{E}[\bar{V}] \leq\left(\bar{V}(0)-\frac{c_{2}}{c_{1}}\right) e^{-c_{1} t}+\frac{c_{2}}{c_{1}} \leq \begin{cases}\bar{V}(0) & \text { if } \bar{V}(0) \geq \frac{c_{2}}{c_{1}} \\ \frac{c_{2}}{c_{1}} & \text { if } \bar{V}(0)<\frac{c_{2}}{c_{1}}\end{cases}
$$

Therefore

$$
\mathbb{E}[\bar{V}] \leq \max \left\{\bar{V}(0), \frac{c_{2}}{c_{1}}\right\}, \forall t
$$

In the long run,

$$
\mathbb{E}[\bar{V}] \leq \frac{c_{2}}{c_{1}}=\frac{\eta^{2}}{2 c_{1}\left(2 a \lambda_{2}-c_{1}\right)}+\frac{b|\operatorname{Tr}(L)|}{2 c_{1} N}+m T \frac{(N-1)}{c_{1} N} .
$$

Notice that the above inequality is valid for all $c_{1} \in\left(0,2 a \lambda_{2}\right)$, of which we look for the minimum over all possible $c_{1}$ 's. Define

$$
\begin{aligned}
\psi_{2}\left(c_{1}\right) & =\frac{\eta^{2}}{2 c_{1}\left(2 a \lambda_{2}-c_{1}\right)}+\frac{b|\operatorname{Tr}(L)|}{2 c_{1} N}+m T \frac{(N-1)}{c_{1} N} \\
& =\left[\frac{\eta^{2}}{4 a \lambda_{2}}+\frac{b|\operatorname{Tr}(L)|}{2 N}+m T \frac{(N-1)}{N}\right] \frac{1}{c_{1}}+\frac{\eta^{2}}{4 a \lambda_{2}} \frac{1}{\left(2 a \lambda_{2}-c_{1}\right)} .
\end{aligned}
$$

By Cauchy-Schwarz inequality, when

$$
\frac{1}{c_{1}}=\frac{1}{2 a \lambda_{2}}\left(1+\sqrt{\frac{c_{4}}{c_{3}}}\right)
$$

$\psi_{2}\left(c_{1}\right)$ attains its minimum

$$
\psi_{2}^{*}=\frac{1}{2 a \lambda_{2}}\left(\sqrt{c_{3}}+\sqrt{c_{4}}\right)^{2} .
$$

Here

$$
c_{3}=\frac{\eta^{2}}{4 a \lambda_{2}}+\frac{b|\operatorname{Tr}(L)|}{2 N}+m T \frac{(N-1)}{N}, c_{4}=\frac{\eta^{2}}{4 a \lambda_{2}} .
$$

Since $(3.15)$ is valid for all $c_{1} \in\left(0,2 a \lambda_{2}\right)$, it holds true that $\mathbb{E}[\bar{V}] \leq \psi_{2}^{*}$ in the long run.

Remark 1. Since the ensemble average of $\bar{V}$ is uniformly bounded in $t$, (time-asymptotic) swarming is achieved in both cases. 
Remark 2. We may expect that if $\bar{V}(0)$ is large, i.e., agents are widely dispersed initially, $\bar{V}$ will decrease and agents get closer from the beginning. If $\bar{V}(0)$ is small compared to the upper bound, $\bar{V}$ may increase first due to the inter-agent repulsion force, but still remains bounded. In Case 1, the speed of swarming depends on $2\left(\kappa+a \lambda_{2}\right)$, i.e., larger algebraic connectivity, stronger attraction and stronger convexity of $\sigma$ facilitate swarming. In Case 2, $2 a \lambda_{2}$ determines the speed.

Remark 3. $\psi_{1}^{*}$ is a tight bound. Supposing that the inequality in Assumption 1 is indeed an equality and graph $\mathcal{G}$ is complete (every two agents directly interact with each other), then $\psi_{1}^{*}$ is exactly the ensemble average of $\bar{V}$ in the long run (See Fig. 3.1(c) for a reference). $\psi_{2}^{*}$ is a looser bound compared to $\psi_{1}^{*}$. Nevertheless, as $\lambda_{2} \rightarrow \infty \psi_{2}^{*}$ approaches the exact swarm size.

Remark 4. The resulting swarm size depends on the inter-individual attraction/repulsion parameters $(a$ and $b)$ and the parameters of the nutrient profile $(\kappa$ and $\eta)$. The noise strength $T$ also plays a role. The dependence of the swarm size on these parameters could be explained as follows: larger attraction (larger a) leads to a smaller swarm size, while larger repulsion (larger $b$ ) leads to a larger swarm size. In Case 1, stronger convexity of $\sigma$ (larger $\kappa$ ) leads to a smaller swarm size. In Case 2, a fast changing landscape (larger $\eta$ ) leads to a larger swarm size. Stronger noise (larger $T$ ) results in a larger swarm in both cases, which is intuitively true.

Remark 5. The algebraic connectivity $\lambda_{2}$ plays a crucial role in determining $\psi_{1}^{*}$ and $\psi_{2}^{*}$. When $N$ is fixed, larger $\lambda_{2}$ leads to smaller bounds. In a complete graph, $\lambda_{2}=N$ and $\operatorname{Tr}(L)=N(N-1)$, in which case

$$
\begin{aligned}
& \lim _{N \rightarrow \infty} \psi_{1}^{*}=\lim _{N \rightarrow \infty}\left[\frac{b(N-1)}{4(\kappa+a N)}+\frac{m T(N-1)}{2 N(\kappa+a N)}\right]=\frac{b}{4 a}, \\
& \lim _{N \rightarrow \infty} \psi_{2}^{*} \\
& =\lim _{N \rightarrow \infty} \frac{1}{2 a N}\left[\sqrt{\frac{\eta^{2}}{4 a N}+\frac{b(N-1)}{2}+\frac{m T(N-1)}{N}}+\sqrt{\frac{\eta^{2}}{4 a N}}\right]^{2} \\
& =\frac{b}{4 a} .
\end{aligned}
$$


This result implies that in a large group, the ultimate bound of swarm size depends only on the interplay between inter-individual attraction and repulsion.

\subsection{Social Foraging Properties}

In this section, we investigate how swarming behavior affects social foraging under Assumption 1 and an additional Lipschitz condition.

Let $F_{i}=(1 / 2)\left\|x_{i}\right\|^{2}$, a function of the distance between agent $i$ and the optimum. Let

$\bar{F}=(1 / N) \sum_{i=1}^{N} F_{i}$ to indicate the social foraging properties: smaller $\bar{F}$ implies a prior group foraging condition. Hence we define successful social foraging for the stochastic system (3.4) as following:

Definition 2. (Social Foraging) System (3.4) achieves a successful social foraging if and only if the ensemble average of the Lyapunov function $\bar{F}$ can be made arbitrarily small.

Assumption 3. (Lipschitz continuity) $\|\nabla \sigma(y)-\nabla \sigma(x)\| \leq \mu\|y-x\|$ for some $\mu \geq \kappa$ and for all $x, y$.

Theorem 2. Suppose that Assumption 1 and Assumption 3 hold. The ensemble average of $\bar{F}$ is uniformly bounded. More precisely,

$$
\begin{aligned}
& \mathbb{E}[\bar{F}] \\
& \leq e^{-2 \kappa t}\left[U(0)+\frac{(\mu-\kappa)}{a \lambda_{2}} \bar{V}(0)\right]+\left[\frac{m T}{2 \kappa N}+\frac{(\mu-\kappa)(b|\operatorname{Tr}(L)|+2 m T(N-1))}{4 a \kappa \lambda_{2} N}\right]\left(1-e^{-2 \kappa t}\right) \\
& +\left|1-\frac{(\mu-\kappa)}{a \lambda_{2}}\right|\left\{e^{-2\left(\kappa+a \lambda_{2}\right) t} \bar{V}(0)+\left[\frac{b|\operatorname{Tr}(L)|}{4 N\left(\kappa+a \lambda_{2}\right)}+\frac{m T(N-1)}{2 N\left(\kappa+a \lambda_{2}\right)}\right]\left[1-e^{-2\left(\kappa+a \lambda_{2}\right) t}\right]\right\} .
\end{aligned}
$$

Here $U:=(1 / 2)\|\bar{x}\|^{2}$. In the long run,

$$
\begin{aligned}
\left.\mathbb{E}[\bar{F}] \leq \phi^{*}=\frac{m T}{2 \kappa N}+\frac{(\mu-\kappa)(b|\operatorname{Tr}(L)|}{4 a \kappa \lambda_{2} N}+2 m T(N-1)\right) \\
+\left|1-\frac{(\mu-\kappa)}{a \lambda_{2}}\right|\left[\frac{b|\operatorname{Tr}(L)|}{4 N\left(\kappa+a \lambda_{2}\right)}+\frac{m T(N-1)}{2 N\left(\kappa+a \lambda_{2}\right)}\right] .
\end{aligned}
$$


Proof. Notice that

$$
\begin{aligned}
\bar{F} & =\frac{1}{N} \sum_{i=1}^{N} \frac{1}{2}\left(e_{i}+\bar{x}\right) \cdot\left(e_{i}+\bar{x}\right) \\
& =\frac{1}{N} \sum_{i=1}^{N} \frac{1}{2}\left\|e_{i}\right\|^{2}+\frac{1}{N} \sum_{i=1}^{N} \frac{1}{2}\|\bar{x}\|^{2}+\frac{1}{N} \sum_{i=1}^{N} e_{i} \cdot \bar{x}=\bar{V}+U
\end{aligned}
$$

According to Equation (3.5) and Ito's lemma,

$$
\begin{aligned}
d U & =d \bar{x} \cdot \bar{x}+\frac{1}{2} d \bar{x} \cdot d \bar{x} \\
& =-\frac{1}{N} \sum_{i=1}^{N} \nabla^{T} \sigma\left(x_{i}\right) \bar{x} d t+\frac{\sqrt{2 T}}{N} \sum_{i=1}^{N} d B_{i}^{T} \bar{x}+\frac{m T}{N} d t \\
& =-\frac{1}{N} \sum_{i=1}^{N} \nabla^{T} \sigma\left(x_{i}\right) x_{i} d t+\frac{1}{N} \sum_{i=1}^{N} \nabla^{T} \sigma\left(x_{i}\right) e_{i} d t+\frac{\sqrt{2 T}}{N} \sum_{i=1}^{N} d B_{i}^{T} \bar{x}+\frac{m T}{N} d t .
\end{aligned}
$$

Since $\sigma(\cdot)$ attains its minimum at the origin, $\nabla \sigma((0,0))=0$. By Assumption 1 and (3.17),

$$
-\frac{1}{N} \sum_{i=1}^{N} \nabla^{T} \sigma\left(x_{i}\right) x_{i} \leq-2 \kappa \bar{F}=-2 \kappa(U+\bar{V}) .
$$

By Assumption 3,

$$
\frac{1}{N} \sum_{i=1}^{N} \nabla^{T} \sigma\left(x_{i}\right) e_{i}=\frac{1}{N} \sum_{i=1}^{N}\left(\nabla^{T} \sigma\left(x_{i}\right)-\nabla^{T} \sigma(\bar{x})\right)\left(x_{i}-\bar{x}\right) \leq \frac{1}{N} \sum_{i=1}^{N} \mu\left\|e_{i}\right\|^{2}=2 \mu \bar{V} .
$$

We have

$$
\begin{aligned}
d U & \leq-2 \kappa(U+\bar{V}) d t+2 \mu \bar{V} d t+\frac{m T}{N} d t+\frac{\sqrt{2 T}}{N} \sum_{i=1}^{N} d B_{i}{ }^{T} \bar{x} \\
& =-2 \kappa U d t+2(\mu-\kappa) \bar{V} d t+\frac{m T}{N} d t+\frac{\sqrt{2 T}}{N} \sum_{i=1}^{N} d B_{i}{ }^{T} \bar{x}
\end{aligned}
$$

Recalling (3.11) and (3.2),

$$
\begin{aligned}
d \bar{V} \leq & -\frac{a \lambda_{2}}{N} \sum_{i=1}^{N}\left\|e_{i}\right\|^{2} d t+\frac{1}{2 N} \sum_{i=1}^{N} \sum_{j=1, j \neq i}^{N} \alpha_{i j} g_{r}\left(\left\|x_{i}-x_{j}\right\|\right)\left\|x_{i}-x_{j}\right\|^{2} d t \\
& -\frac{1}{N} \sum_{i=1}^{N} \nabla^{T} \sigma\left(x_{i}\right) e_{i} d t+\frac{\sqrt{2 T}}{N} \sum_{i=1}^{N} d B_{i}^{T} e_{i}+m T \frac{(N-1)}{N} d t \\
\leq & -2 a \lambda_{2} \bar{V} d t-2 \kappa \bar{V} d t+\frac{b|T r(L)|}{2 N} d t+m T \frac{(N-1)}{N} d t+\frac{\sqrt{2 T}}{N} \sum_{i=1}^{N} d B_{i}^{T} e_{i} .
\end{aligned}
$$


Define

$$
W=U+\frac{(\mu-\kappa)}{a \lambda_{2}} \bar{V}
$$

By (3.19) and (3.21), we obtain

$$
\begin{aligned}
d W \leq & -2 \kappa U d t+\left[2(\mu-\kappa)-\frac{(\mu-\kappa)}{a \lambda_{2}}\left(2 \kappa+2 a \lambda_{2}\right)\right] \bar{V} d t+\frac{m T}{N} d t \\
& +\frac{(\mu-\kappa)}{a \lambda_{2}}\left[\frac{b|T r(L)|}{2 N}+m T \frac{(N-1)}{N}\right] d t+\frac{\sqrt{2 T}}{N} \sum_{i=1}^{N} d B_{i}^{T} \bar{x}+\frac{(\mu-\kappa)}{a \lambda_{2}} \frac{\sqrt{2 T}}{N} \sum_{i=1}^{N} d B_{i}^{T} e_{i} \\
= & -2 \kappa W d t+\frac{m T}{N} d t+\frac{(\mu-\kappa)(b|T r(L)|+2 m T(N-1))}{2 a \lambda_{2} N} d t+\frac{\sqrt{2 T}}{N} \sum_{i=1}^{N} d B_{i}^{T} \bar{x} \\
& +\frac{(\mu-\kappa)}{a \lambda} \frac{\sqrt{2 T}}{N} \sum_{i=1}^{N} d B_{i}^{T} e_{i} .
\end{aligned}
$$

Applying Ito's lemma to $e^{2 \kappa t} W$,

$$
\begin{aligned}
d\left(e^{2 \kappa t} W\right)=e^{2 \kappa t} d W+2 \kappa e^{2 \kappa t} W d t \leq\left[\frac{m T}{N}\right. & \left.+\frac{(\mu-\kappa)(b|\operatorname{Tr}(L)|+2 m T(N-1))}{2 a \lambda_{2} N}\right] e^{2 \kappa t} d t \\
& +\frac{\sqrt{2 T}}{N} e^{2 \kappa t}\left[\sum_{i=1}^{N} d B_{i}{ }^{T} \bar{x}+\frac{(\mu-\kappa)}{a \lambda_{2}} \sum_{i=1}^{N} d B_{i}{ }^{T} e_{i}\right] .
\end{aligned}
$$

Integrating both sides and taking ensemble average, we get

$$
\mathbb{E}[W] \leq e^{-2 \kappa t} W(0)+\left[\frac{m T}{2 \kappa N}+\frac{(\mu-\kappa)(b|\operatorname{Tr}(L)|+2 m T(N-1))}{4 a \kappa \lambda_{2} N}\right]\left(1-e^{-2 \kappa t}\right) .
$$

By (3.12) and (3.17),

$$
\begin{aligned}
& \mathbb{E}[\bar{F}]=\mathbb{E}[W]+\left[1-\frac{(\mu-\kappa)}{a \lambda_{2}}\right] \mathbb{E}[V] \\
& \leq e^{-2 \kappa t}\left[U(0)+\frac{(\mu-\kappa)}{a \lambda_{2}} \bar{V}(0)\right]+\left[\frac{m T}{2 \kappa N}+\frac{(\mu-\kappa)(b|\operatorname{Tr}(L)|+2 m T(N-1))}{4 a \kappa \lambda_{2} N}\right]\left(1-e^{-2 \kappa t}\right) \\
& +\left|1-\frac{(\mu-\kappa)}{a \lambda_{2}}\right|\left\{e^{-2\left(\kappa+a \lambda_{2}\right) t} \bar{V}(0)+\left[\frac{b|\operatorname{Tr}(L)|}{4 N\left(\kappa+a \lambda_{2}\right)}+\frac{m T(N-1)}{2 N\left(\kappa+a \lambda_{2}\right)}\right]\left[1-e^{-2\left(\kappa+a \lambda_{2}\right) t}\right]\right\} .
\end{aligned}
$$

In the long run,

$$
\begin{aligned}
\mathbb{E}[\bar{F}] \leq \phi^{*}=\frac{m T}{2 \kappa N}+\frac{(\mu-\kappa)(b|\operatorname{Tr}(L)|+2 m T(N-1))}{4 a \kappa \lambda_{2} N} & \\
+\left|1-\frac{(\mu-\kappa)}{a \lambda_{2}}\right| & {\left[\frac{b|\operatorname{Tr}(L)|}{4 N\left(\kappa+a \lambda_{2}\right)}+\frac{m T(N-1)}{2 N\left(\kappa+a \lambda_{2}\right)}\right] . }
\end{aligned}
$$


Remark 6 . Theorem 2 shows that social foraging is successful since for either $b=0$ (i.e., no repulsive force) or $b / a \sim 1 / N$ (i.e., increasing relative attractive force with increasing group size), distances between agents and the optimal location can be made arbitrarily small. The speed of group foraging depends on $\kappa$ and $a \lambda_{2}$. Agents move faster towards the optimum in a field with larger gradients, larger inter-agent attraction, and when graph connectivity is strong.

Remark 7. $\phi^{*}$ is exact when $\kappa=\mu$ (See Fig. 1(e) for a reference). When $\kappa<\mu$, the tightness of $\phi^{*}$ depends on detailed information of $\nabla \sigma(\cdot)$.

Remark 8. $\phi^{*}$ implies the ultimate social foraging properties. It is determined by interindividual attraction/repulsion ( $a$ and $b)$, gradient of the nutrient profile $(\kappa$ and $\mu)$, algebraic connectivity $\lambda_{2}$, the number of agents $N$, and the noise strength $T$. Larger repulsion (larger $b$ ) and stronger noise (larger $T$ ) impedes social foraging. Larger gradient (large $\kappa$ and smaller $\mu$ ), stronger algebraic connectivity (larger $\lambda_{2}$ ), larger group size (larger $N$ ) and stronger attraction (larger $a$ ) makes for foraging success. The dependence of $\phi^{*}$ on $a, \lambda_{2}$ and $N$ demonstrates the benefit of swarming behavior. Furthermore, when $\mathcal{G}$ is complete,

$$
\begin{aligned}
\lim _{N \rightarrow \infty} \phi^{*}= & \lim _{N \rightarrow \infty}\left\{\frac{m T}{2 \kappa N}+\frac{(\mu-\kappa)(N-1)}{2 \kappa a N}\left(\frac{b}{2}+\frac{m T}{N}\right)\right. \\
& \left.+\left|1-\frac{(\mu-\kappa)}{a N}\right|\left[\frac{b(N-1)}{4(\kappa+a N)}+\frac{m T(N-1)}{2 N(\kappa+a N)}\right]\right\} \\
= & \frac{\mu b}{4 \kappa a} .
\end{aligned}
$$

In a large group, the ultimate foraging properties depends only on two factors: the interplay between inter-individual attraction and repulsion, and the gradient of the nutrient profile. As this limit is not a function of $T$, the effect of noise disappears altogether.

\subsubsection{Noise Reduction Property}

Let us now compare the performance of a swarming discipline with individual foraging (i.e., no interaction exists between agents). In this scenario,

$$
d x_{i}=-\nabla \sigma\left(x_{i}\right) d t+\sqrt{2 T} d B_{i}
$$


Let $H_{i}=(1 / 2)\left\|x_{i}\right\|^{2}$. We have

$$
\begin{aligned}
d H_{i} & =d x_{i} \cdot x_{i}+(1 / 2) d x_{i} \cdot d x_{i} \\
& =-\nabla^{T} \sigma\left(x_{i}\right) x_{i} d t+\sqrt{2 T} d B_{i}^{T} x_{i}+m T d t \\
& \geq-2 \mu H_{i} d t+m T d t+\sqrt{2 T} d B_{i}^{T} x_{i} .
\end{aligned}
$$

Resembling the proof of Theorem 2, we conclude that:

$$
\mathbb{E}\left[H_{i}\right] \geq e^{-2 \mu t} H_{i}(0)+\frac{m T}{2 \mu}\left(1-e^{-2 \mu t}\right),
$$

and in the long run,

$$
\liminf \operatorname{si}_{t}\left[H_{i}(t)\right] \geq \frac{m T}{2 \mu}
$$

The noise reduction property by swarming in social foraging can be formalized as follows.

Corollary 1. Suppose that graph $\mathcal{G}$ is complete. If either $b=0$ or $b / a \sim 1 / N$, there exists an $N^{*}>0$ and a $t^{*}<\infty$ such that $\mathbb{E}[\bar{F}]<\mathbb{E}\left[H_{i}\right]$ for all $t>t^{*}$.

Proof. Given that $\mathcal{G}$ is complete, $\lambda_{2}=N$ and $|\operatorname{Tr}(L)|=N(N-1)$. The ultimate upper bound obtained in Theorem 2 is

$$
\phi^{*}=\frac{m T}{2 \kappa N}+\frac{(\mu-\kappa)(N-1)}{2 \kappa a N}\left(\frac{b}{2}+\frac{m T}{N}\right)+\left|1-\frac{(\mu-\kappa)}{a N}\right|\left[\frac{b(N-1)}{4(\kappa+a N)}+\frac{m T(N-1)}{2 N(\kappa+a N)}\right] .
$$

If $b=0$ or $b / a \sim 1 / N, \phi^{*}$ is strictly monotonically decreasing to 0 in the group size. Thus, there exists an $N^{*}>0$ such that

$$
\phi^{*}<\frac{m T}{2 \mu} \leq \lim \inf _{t} E\left[H_{i}\right]
$$

for all $N>N^{*}$. Since the upper bound of $\mathbb{E}[\bar{F}]$ converges to $\phi^{*}$ in $t$, there exists a $t^{*}<\infty$ such that $\mathbb{E}[\bar{F}(t)]<\mathbb{E}\left[H_{i}(t)\right]$ for all $t>t^{*}$.

Remark 9 . This result can be extended to the case that $\lambda_{2} \sim N$ while $\mathcal{G}$ need not be complete. 
Corollary 1 demonstrates that when $b=0$ (i.e., no repulsive force) or $b / a \sim 1 / N$ (i.e., increasing relative attractive force with increasing group size) a swarming discipline for foraging exhibits better performance than individual foraging. For convex, smooth nutrient profiles a large realization of noise leads to a large perturbation of the gradient which in turn induces a trajectory that is too dissimilar from that of the group. The swarming discipline forces individuals to discard large noise perturbations of the gradient in their effort to maintain cohesion. As a result, the group trajectories are less affected by noise. It is important to emphasize here that this noise reduction effect is not related to averaging.

\subsection{Simulation Examples}

In this section, we provide some simulation examples to relax the assumptions used in the theory developed in the preceding sections.

\subsubsection{Quadratic Resource Profile}

First we consider a quadratic resource profile satisfying Assumption 1 and 3. For the example, we choose an $m=2$ dimensional space for ease of visualization of the results and use the region $[50,150] \times[50,150]$ in the space. In the simulations we use a complete network with $N=15$ individuals. The attraction/repulsion function $g(y)=-y\left[a-c \exp \left(-\|y\|^{2}\right)\right]$ is adopted with parameters $a=0.01$ and $c=0.05$. The equilibrium distance for $g(\cdot)$ is $\rho=\sqrt{\ln (c / a)}=1.27$. Notice that $g_{r}(\|y\|)\|y\|^{2}=c \exp \left(-\|y\|^{2}\right)\|y\|^{2} \leq c e^{-1} \simeq 0.136=$ b. Initially, all the agents are distributed randomly in the $[50,200] \times[50,200]$ interval. We consider noise level $T=2$. Simulations are run for agents with and without mutual interactions. This allows us to analyze how swarming affect foraging in the presence of noise. Note that in the no-interaction case, each individual could be regarded as a single foraging group $(N=1)$.

The particular nutrient profile we consider in this example is $\sigma(y)=0.01\|y\|^{2}$, whose gradient is given by $\nabla \sigma(y)=0.02 y$. Simulation running time is $500 \mathrm{~s}$. 


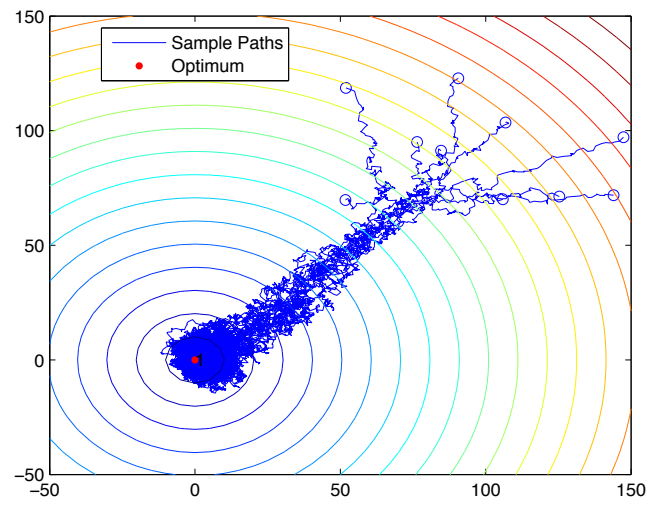

(a) Sample paths with swarming.

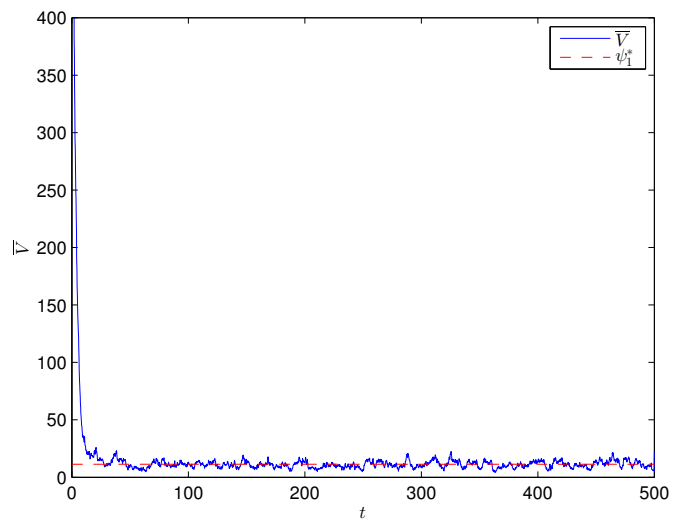

(c) $\bar{V}$ (swarming).

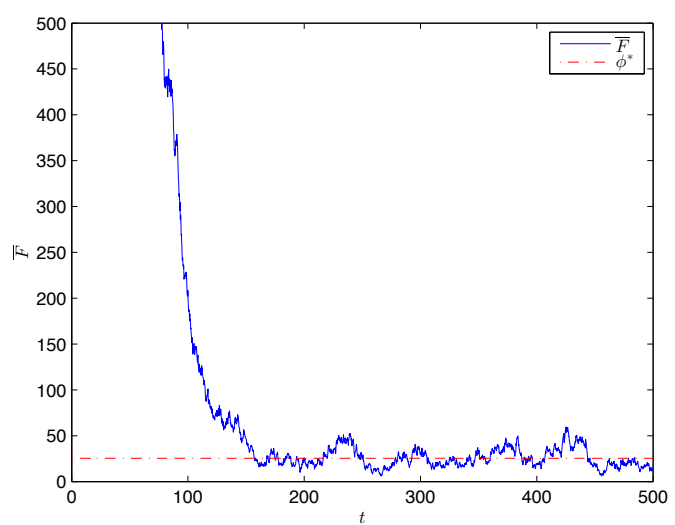

(e) $\bar{F}$ (swarming).

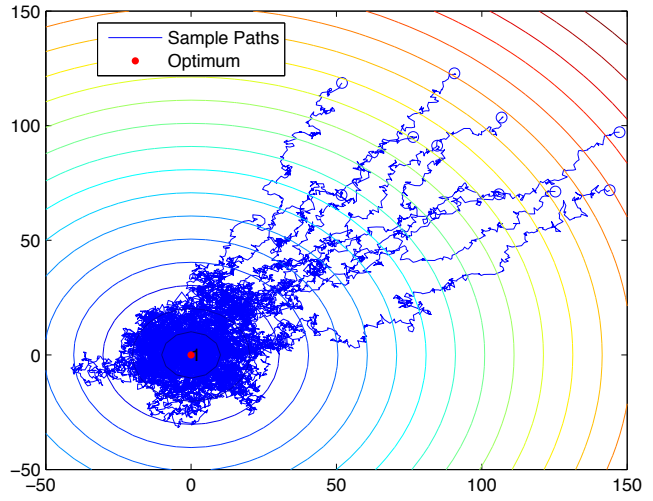

(b) Independent sample paths.

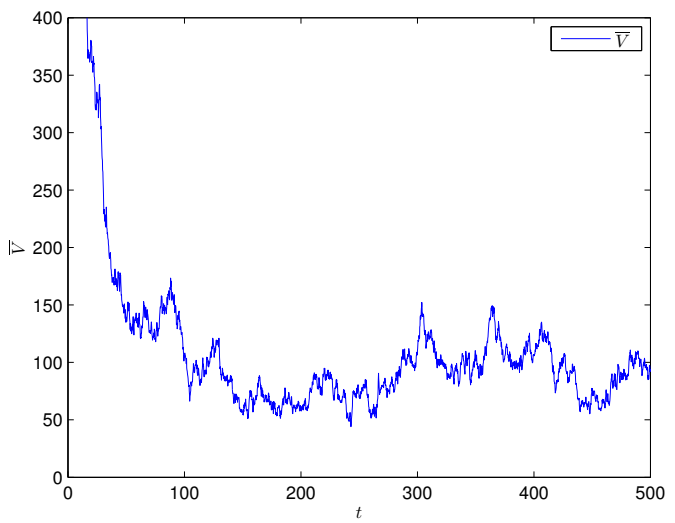

(d) $\bar{V}$ (no interaction).

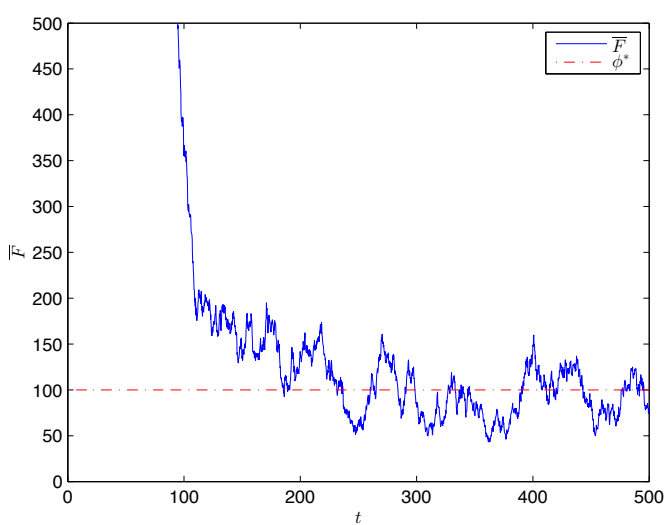

(f) $\bar{F}$ (no interaction).

Figure 3.1: Social foraging with and without mutual interactions with quadratic resource profile. 
Fig. 3.1(a)(b) plots the trajectories of the individuals. With mutual interactions, the agents form a cohesive group and collectively move towards the global minimum (nutrient center). By contrast, individuals' movements look more random without mutual interactions, and they cannot get as close to the optimum as when they form a swarm. This simulation example suggests that swarming (for social foraging) provides a noise reduction effect.

Cohesive properties are further illustrated in Fig. 3.1(c)(d) that plots the average fluctuations $\bar{V}$. When agents interact, $\bar{V}$ decreases at first and remains at a level around $\psi_{1}^{*}$ as expected. By contrast, when agents do not interact, fluctuations stay large, and the foraging group is dispersed.

Foraging properties are shown in Fig. 3.1(e)(f). In the presence of mutual interaction, $\bar{F}$ decreases to a level around $\phi^{*}$ which is lower than when individuals do not interact.

\subsubsection{Non-convex Resource Profile}

It was assumed that resource function $\sigma(\cdot)$ is strongly convex in Section 3.5 and in the above example. Our results are likely to hold for non-convex nutrient profiles as well. To illustrate, we consider nutrient profile $\sigma(y)=\ln \left(\|y\|^{2}+1\right)$, whose gradient is given by

$$
\nabla \sigma(y)=2 y /\left(\|y\|^{2}+1\right)
$$

Notice that $\|\nabla \sigma(y)\|$ first increases and then decreases in $\|y\|$. Other settings stay the same as in Section 3.6.1 except that running time is 7500 s.

In this case, agents are still able to reach and stay close to the optimum when they form a swarm. On the contrary, without mutual interactions their motions are almost random. In Fig. 3.2(d) we observe that even when an individual gets close to the nutrient center, it will go away because of noise. Therefore, swarming is critical for foraging success in this scenario. 


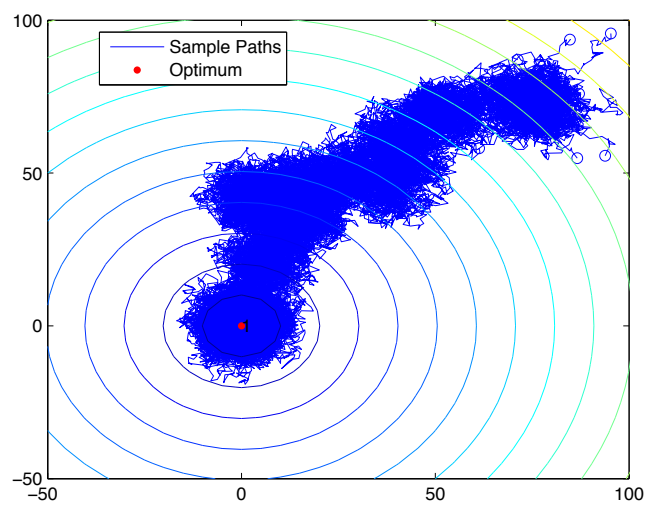

(a) Sample paths with swarming.

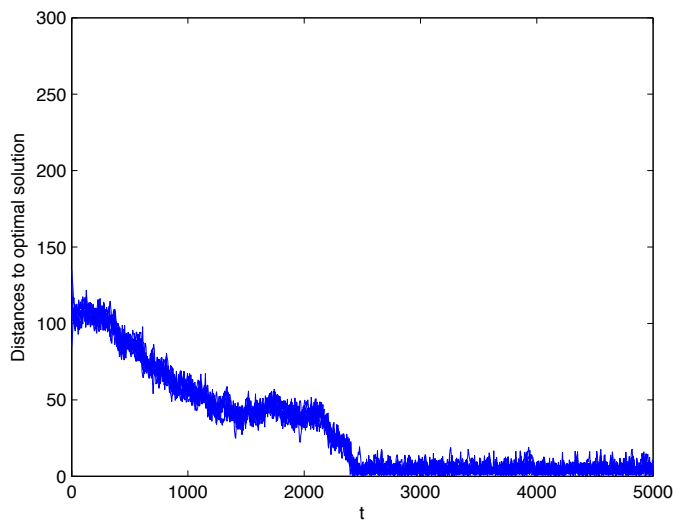

(c) Distance to optimum with swarming.

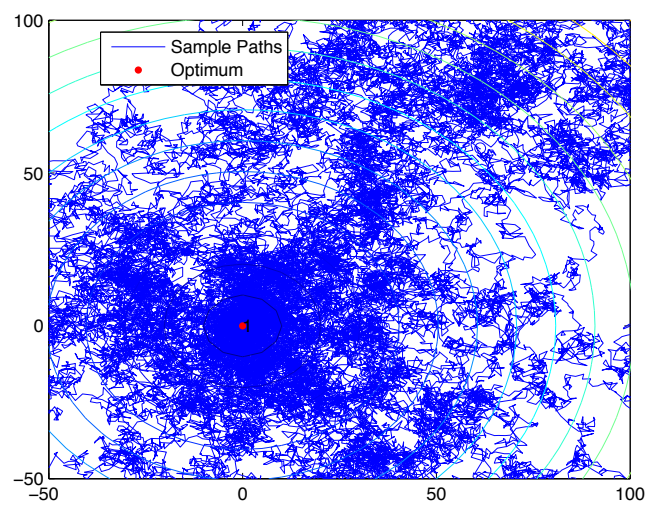

(b) Independent sample paths.

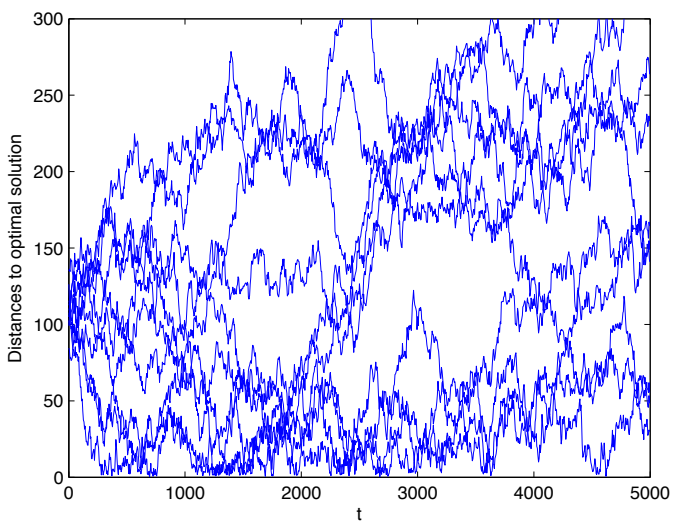

(d) Distance to optimum without interaction.

Figure 3.2: Social foraging with and without mutual interactions with non-quadratic resource profile. 


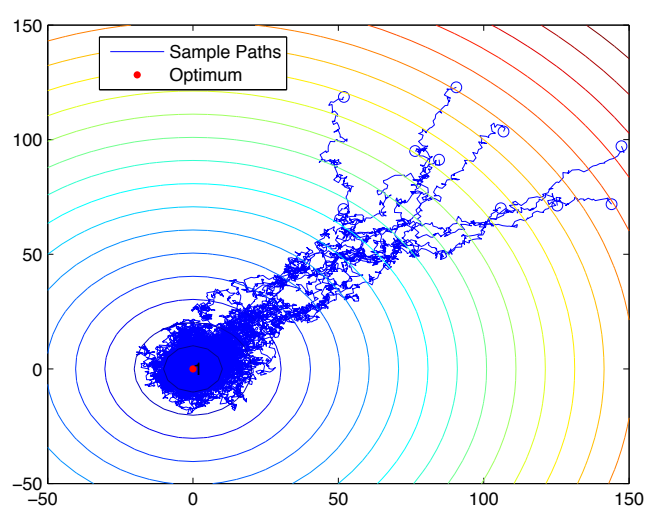

(a) Sample paths within an incomplete graph.

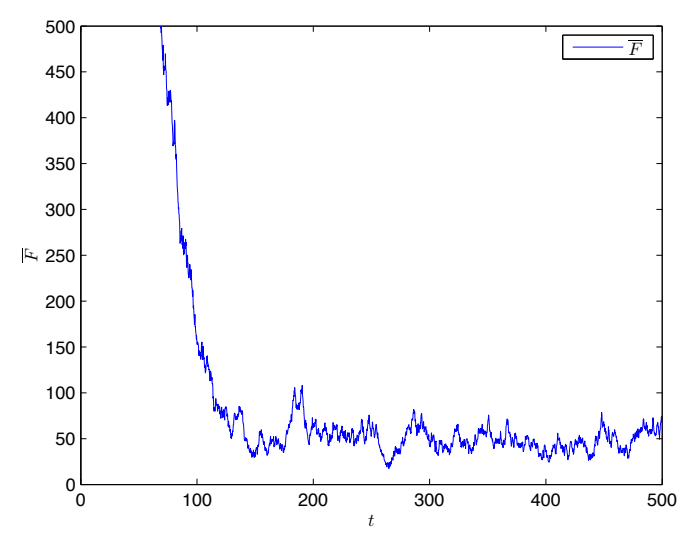

(b) $\bar{F}$ (incomplete graph).

Figure 3.3: Social foraging within an incomplete graph with quadratic resource profile.

\subsubsection{Incomplete Graph}

We have been running simulations within a complete network. However, in a large group, an individual usually communicate with a few neighbors. In this example we consider the case in which each agent communicates with his 6 nearest neighbors. Our setting here agrees with Ballerini et al. [4] where evidence was given to prove that collective animal behavior (swarming) depends on topological rather than metric distance.

Aside from network connectivity we follow the settings used in Section 3.6.1. Compared with Fig. 3.1(a)(b), we find that the group foraging behavior shown in Fig. 3.3 falls between the previous two. Agents form a swarm that is more cohesive than in the interaction-free case. Nonetheless it is more dispersed than in a complete network.

\subsection{Conclusions of This Chapter}

There is empirical evidence that swarming is a useful strategy for social foraging in a noisy environment. To our knowledge, there is no mathematical formalization of noise reduction properties of swarming in social foraging. In this chapter we develop a mathematical model for analyzing the benefits of social foraging in a noisy environment. We identify conditions 
on the nutrient profile ensuring that local agent actions will lead to cohesive foraging. For convex, smooth nutrient profiles we formalize the way in which swarming for social foraging is better at handling the effects of noise when compared to the average of individual foraging strategies. Under a swarming discipline, observational noise realizations that induce trajectories differing too much from the group average are likely to be discarded because of each individual's need to maintain cohesion. As a result, the group trajectories are less affected by noise. In a limited test-bed we relax the main assumption in our analysis. The results indicate that the noise reduction properties of swarming in social foraging are robust to inter-agent communication constraints and non-convex nutrient profiles. These results suggest that swarming-like approaches for the control of networked agents may provide an additional level of robustness. 


\section{CHAPTER 4}

\section{A Flocking-based Approach for Distributed Stochastic Optimization}

Inspired by the forementioned theory on the noise reduction property of social foraging, in this chapter, we consider a distributed computing algorithmic scheme for stochastic optimization which relies on modest communication requirements amongst processors and most importantly, does not require synchronization. Specifically, we analyze a scheme with $N>1$ independent threads implementing each a stochastic gradient algorithm. The threads are coupled via a perturbation of the gradient (with attractive and repulsive forces) in a similar manner to mathematical models of flocking, swarming and other group formations found in nature with mild communication requirements. When the objective function is convex, we show that a flocking-like approach for distributed stochastic optimization provides a noise reduction effect similar to that of a centralized stochastic gradient algorithm based upon the average of $N$ gradient samples at each step. The distributed nature of flocking makes it an appealing computational alternative. We show that when the overhead related to the time needed to gather $N$ samples and synchronization is not negligible, the flocking implementation outperforms a centralized stochastic gradient algorithm based upon the average of $N$ gradient samples at each step. When the objective function is not convex, the flocking-based approach seems better suited to escape locally optimal solutions due to the repulsive force which enforces a certain level of diversity in the set of candidate solutions. Here again, we show that the noise reduction effect is similar to that associated to the centralized stochastic 
gradient algorithm based upon the average of $N$ gradient samples at each step.

\subsection{Introduction}

Swarms, flocks and other group formations can be found in nature in many organisms ranging from simple bacteria to mammals (see $[72,75,80]$ for references). Such collective and coordinated behavior is believed to be effective for avoiding predators and/or for increasing the chances of finding food (foraging) (see $[38,79]$ ). In this chapter we introduce a novel distributed scheme for stochastic optimization wherein multiple independent computing threads implement each a stochastic gradient algorithm which is further perturbed by repulsive and attractive terms (a function of the relative distance between solutions). Thus, the updating of individual solutions is coupled in a similar manner to mathematical models of swarming, flocking and other group formations found in nature (see Gazi and Passino [33]). We show that this coupling endows the flocking scheme with an important robustness property as noise realizations that induce trajectories differing too much from the group average are likely to be discarded.

The performance of the single-thread stochastic gradient algorithm is highly sensitive to noise. Thus, there is a literature on estimation techniques leading to better gradient estimation often involving increasing sample size (see $[27,89]$ for a survey of gradient estimation techniques). When sampling is undertaken in parallel, synchronization is needed to execute the tasks that can not be executed in parallel. The speed-up obtained by parallel sampling and centralized gradient estimation is limited by overhead related to (i) time spent gathering samples (which could be significant for example in the simulation of complex systems) and (ii) synchronization. In contrast, the noise reduction obtained in a flocking-based approach with $N>1$ threads does not require synchronization since each thread only needs the information on the current solution identified by neighboring threads (where the notion of neighborhood is related to a given network topology). When sampling times are not negligible and exhibit large variation, synchronization may cause significant overhead so that real-time performance of stochastic gradient algorithm based upon the average of $N$ sam- 
ples obtained in parallel is highly affected by large sampling time variability. In contrast, the real-time performance of a flocking-based implementation with $N>1$ threads may be superior as each thread can asynchronously update its solution based upon a small sample size and still reap the benefits of noise reduction stemming from the flocking discipline.

To illustrate the noise reduction property, consider the minimization of the function $f(x)=\ln \left(\|x\|^{2}+1\right)$ where $x \in \mathbb{R}^{2}$. The unique optimal solution is $x^{*}=(0,0)$. Suppose that the gradient $\nabla f(x)$ is observed with noise so that the basic iteration in a stochastic gradient descent algorithm can be written as:

$$
x(k+1)=x(k)+\Gamma(k)(-\nabla f(x(k))+\varepsilon(k)),
$$

where $\Gamma(k)>0$ is the step size, and the collection $\{\varepsilon(k): k>0\}$ is i.i.d (independent and identically distributed) with mean zero and variance $\sigma^{2}$. The stochastic gradient method with constant step size $\Gamma(k)=0.02$ and normally distributed noise with $\sigma^{2}=0.18$ is unable to approximate the optimal solution given the large magnitude of noise (relative to the gradient). One solution to this conundrum is to implement an improved version based upon the average of $N=10$ samples of the gradient at each step. Then, the variance of noise is reduced to $\sigma^{2} / N=0.018$. Supposing that each step takes $0.02 s$, the performance of this approach is shown in Figure 4.1(a) ${ }^{1}$.

In this dissertation we advocate a different tack. We introduce an additional perturbation to the gradient so that the basic iteration for thread $i$ is:

$$
x(i, k+1)=x(i, k)+\tilde{\Gamma}(i, k)\left[-\nabla f(x(i, k))+\varepsilon(i, k)-\sum_{j=1, j \neq i}^{N} \alpha_{i j} \nabla_{x(i)} J\left(\left\|x(i, k)-x\left(j_{i}, k\right)\right\|\right)\right],
$$

where $J\left(\left\|x(i, k)-x\left(j_{i}, k\right)\right\|\right)$ is the flocking potential between threads $i$ and $j . \alpha_{i j}=1$ if thread $i$ has access to the current solution $x\left(j^{i}, k\right)$ by thread $j$ and $\alpha_{i j}=0$ otherwise. The term $\nabla_{x(i)} J\left(\left\|x(i, k)-x\left(j^{i}, k\right)\right\|\right)$ is a combination of repulsive and attractive "forces" depending upon the relative distance $\left\|x(i, k)-x\left(j^{i}, k\right)\right\|$ (see Gazi and Passino [30] for reference).

The performance of the flocking-discipline of $N=10$ fully connected threads (again with constant step size $\tilde{\Gamma}(i, k)=0.02$ and $\left.\sigma^{2}=0.18\right)$ is depicted in Figure 4.1(b). The

\footnotetext{
${ }^{1}$ In this illustration example we assume zero variance among sampling times.
} 
performance of the flocking scheme (as measured by the average solution) can be seen to be comparable with the scheme based upon the average of $N=10$ samples at each step (see Figure 4.1(b)). The times needed for the solutions identified by each scheme to reach the ball $B_{0.1}\left(x^{*}\right)=\left\{x \in \mathbb{R}^{2} \mid\left\|x-x^{*}\right\|<0.1\right\}$ are fairly similar. For a total of 100 sample paths the mean time and standard deviation of the centralized scheme and the flocking scheme are (992.6, 1454.6) and $(969.1,1223.4)$, respectively.

This noise reduction effect can be succinctly explained as follows. Under a flocking discipline, noise realizations that induce trajectories differing too much from the group average are likely to be discarded because of the attractive potential effect on each individual thread which leads to cohesion. The noise reduction enabled by a flocking-discipline is fundamentally different from that associated with averaging of independent gradient samples.

Our work is related to the extensive literature in stochastic approximation method dating to Robbins and Monro [82] and Kiefer and Wolfowitz [50]. These work includes the analysis of convergence (conditions for convergence, rates of convergence, proper choice of step size) in the context of diverse noise models (see Kushner and Yin [53]). Recently there has been considerable interest in parallel or distributed implementation of stochastic gradient algorithms (see $[16,60,90,98,104]$ for examples). However, they mainly aim at minimizing a sum of convex functions which is different from our objective.

Our work is also linked with population-based algorithms for simulation-based optimization. In these approaches, at every iteration, the quality of each solution in the population is evaluated and a new population of solutions is randomly generated according to a given rule designed to achieve an acceptable trade-off between "exploration" and "exploitation" effort. Recent efforts have focused on model-based algorithms (see Hu et al. [44]) which differ from population-based approaches in that candidate solutions are generated at each iteration by sampling from a "model" which is a probability distribution over the solution space. The basic idea is to modify the model based on the sampled solutions in order to bias the future search towards regions containing high quality solutions (see $\mathrm{Hu}$ [43] for a recent survey). These approaches are inherently centralized in that the updating of populations (or models) 

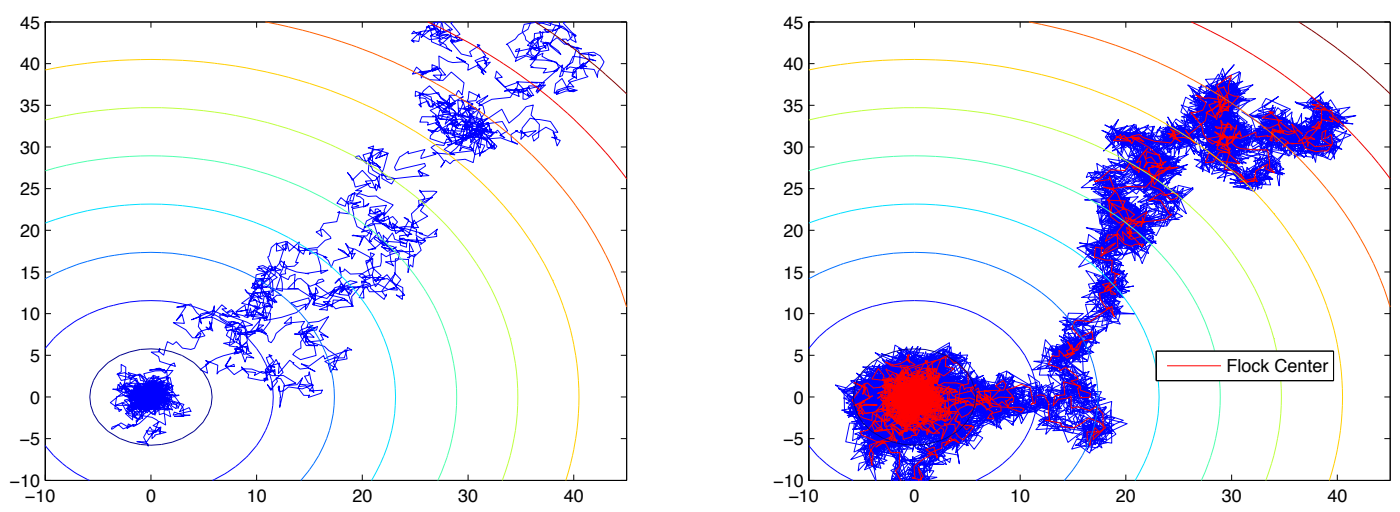

(a) Sample paths for sample average scheme with (b) Sample paths with flocking discipline ( $N$ $N$ samples. threads).
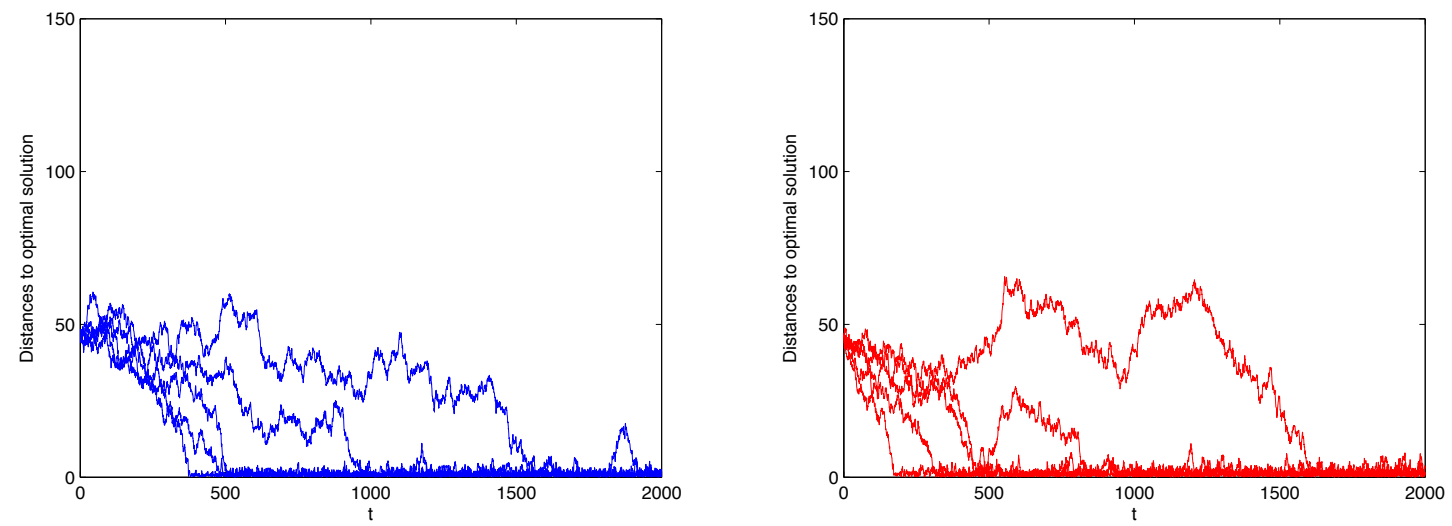

(c) Distance to optimum for sample average (d) Distance between the average flocking solution scheme with $N$ samples. and the optimum.

Figure 4.1: Performance comparison between the scheme based upon the average of $N=10$ gradient samples per step and the flocking-based approach with 10 fully connected threads. 
is undertaken after the quality of all candidate solutions is evaluated.

The structure of this chapter is as follows. Section 4.2 introduces the optimization problem of interest. In Section 4.3, we perform cohesion analysis of the flocking-based approach. Section 4.4 formalizes the noise reduction properties of the flocking-based algorithmic scheme for convex optimization. In Section 4.5, we apply the flocking-based algorithm to the optimization of general non-convex functions. Section 4.6 concludes the chapter.

\subsection{Setup}

\subsubsection{Preliminaries}

In the analysis of this chapter we shall make use of certain graph theoretic concepts which we briefly review below. A graph $\mathcal{G}$ is a pair $(\mathcal{V}, \mathcal{E})$, where $\mathcal{V}$ is a set of vertices and $\mathcal{E}$ is a subset of $\mathcal{V} \times \mathcal{V}$ called edges. A graph $(\mathcal{V}, \mathcal{E})$ is called undirected if $(i, j) \in \mathcal{E} \Leftrightarrow(i, j) \in \mathcal{E}$. The adjacency matrix $A=\left[\alpha_{i j}\right] \in \mathbb{R}$ of a graph is a matrix with nonzero elements satisfying the property $\alpha_{i j}>0 \Leftrightarrow(i, j) \in \mathcal{E}$. Self-joining edges are excluded, i.e., $\alpha_{i i}=0, \forall i$. The Laplacian matrix $L$ associated with a graph $(\mathcal{V}, \mathcal{E})$ is defined as $L=\left[l_{i j}\right]$, where $l_{i i}=\sum_{j} \alpha_{i j}$ and $l_{i j}=-\alpha_{i j}$ where $i \neq j$. For an undirected graph, the Laplacian matrix is symmetric positive semi-definite (see Godsil and Royle [36]).

\subsubsection{Problem Statement}

We consider the problem

$$
\min _{x \in \mathbb{R}^{m}} f(x)
$$

where $f: \mathbb{R}^{m} \rightarrow \mathbb{R}$ is a differentiable function that is not available in closed form. To solve this problem, a black-box noisy simulation model is used. In this context, noise can have many sources such as modeling and discretization error, incomplete convergence, and finite sample size for Monte-Carlo methods (see for instance Kleijnen [51]). Assume that we have $N$ computing threads that can generate gradient samples in parallel. Every gradient sample 
is subject to i.i.d noise $\varepsilon \in \mathbb{R}^{m}$ of mean zero and variance $\sigma^{2}$ in each dimension.

In the rest of this section, we present two algorithms for solving the problem. First, we introduce a centralized stochastic gradient-descent algorithm. Then we propose the flockingbased approach. In both cases, we assume that the step size is a constant value $(\Gamma$ and $\tilde{\Gamma}$, respectively).

\section{A Centralized Algorithm}

A centralized stochastic gradient-descent algorithm is of the form:

$$
x(k+1)=x(k)+\Gamma u(k), \quad k \in \mathbb{N}
$$

where $u(k)=-\nabla f(x(k))+\epsilon(k)$, with $\epsilon(k)$ being the random simulation noise. Assume that sampling takes place through $N$ parallel computing threads, where each thread contributes one sample in a single step. Then, $\epsilon(k)$ is given by an average of $N$ i.i.d random vectors: $\epsilon(k)=(1 / N) \sum_{i=1}^{N} \varepsilon(i)$. Each $\varepsilon(i)$ has $m$ i.i.d components of mean zero and variance $\sigma^{2}$.

In what follows, we approximate the discrete-time process (4.2) by a continuous-time system. Let $\Delta t(k)$ be the time needed to gather $N$ samples for calculating $u(k)$. Then in continuous-time $t, x(k)$ is obtained at $t(k)=\sum_{l<k} \Delta t(l)$. Denote by $x_{t}$ be the identified solution at time $t$. We get $x_{t(k)}=x(k)$ and $x_{t}=x_{t(k-1)}$ for all $t \in(t(k-1), t(k))$, i.e., the continuous-time solution changes only upon updates. By (4.2),

$$
x_{t}=x_{0}-\sum_{t(l)<t} \nabla f\left(x_{t(l)}\right) \Gamma+\sum_{t(l)<t} \epsilon(l) \Gamma .
$$

For ease of analysis, we define a new variable $y_{t}:=x_{t / \Gamma}$. It follows that

$$
\begin{aligned}
y_{t} & =x_{0}-\sum_{t(l)<t / \Gamma} \nabla f\left(x_{t(l)}\right) \Gamma+\sum_{t(l)<t / \Gamma} \epsilon(l) \Gamma \\
& =y_{0}-\sum_{\Gamma t(l)<t} \nabla f\left(y_{\Gamma t(l)}\right) \Gamma+\sum_{t(l)<t / \Gamma} \epsilon(l) \Gamma .
\end{aligned}
$$

Assume that all $\Delta t(k)$ 's are i.i.d with mean $\mathbb{E}(\Delta t(k))=\Delta t$, and let $n_{t}$ be the cardinality of $\{l: \Gamma t(l)<t\}$. By the (strong) law of large numbers, for small $\Gamma>0$,

$$
\frac{\Gamma n_{t}}{t}=\frac{n_{t}}{t / \Gamma} \simeq \frac{1}{\Delta t}, \text { and } \frac{n_{t}}{t} \gg 0 .
$$


Hence,

$$
\sum_{\Gamma t(l)<t} \nabla f\left(y_{\Gamma t(l)}\right) \Gamma=\frac{\Gamma n_{t}}{t}\left[\sum_{\Gamma t(l)<t} \nabla f\left(y_{\Gamma t(l)}\right) \frac{t}{n_{t}}\right] \simeq \frac{\Gamma n_{t}}{t} \int_{0}^{t} \nabla f\left(y_{t}\right) d t \simeq \frac{1}{\Delta t} \int_{0}^{t} \nabla f\left(y_{t}\right) d t .
$$

Note that $\sum_{t(l)<t / \Gamma} \epsilon(l) \Gamma$ has mean zero and variance $\Gamma^{2} n_{t} \sigma^{2} / N$ in each dimension. In light of (4.3),

$$
\frac{\Gamma^{2} n_{t} \sigma^{2}}{N}=\frac{\Gamma n_{t}}{t} \Delta t \frac{\Gamma \sigma^{2} t}{N \Delta t} \simeq \frac{\Gamma \sigma^{2}}{N \Delta t} t
$$

We have

$$
\sum_{t(l)<t / \Gamma} \epsilon(l) \Gamma \simeq \sigma \sqrt{\frac{\Gamma}{N \Delta t}} B_{t}
$$

where $B_{t}$ is the standard $m$-dimensional Brownian motion.

Define $\gamma=1 / \Delta t$ and $\tau_{N}=\sigma \sqrt{\Gamma \Delta t / N}$. Then $y_{t}$ approximately satisfies the following stochastic Ito integration:

$$
y_{t}=y_{0}-\gamma \int_{0}^{t} \nabla f\left(y_{t}\right) d t+\int_{0}^{t} \tau_{N} \gamma d B_{t}
$$

which is usually written in its differential form:

$$
d y_{t}=-\nabla f\left(y_{t}\right) \gamma d t+\tau_{N} \gamma d B_{t}
$$

\section{A Flocking-based Algorithm}

A flocking-based implementation also has $N$ computing threads. In contrast to the centralized approach, each thread $i$ independently implement a stochastic gradient algorithm based on only one sample at each step:

$$
x(i, k+1)=x(i, k)+\tilde{\Gamma} u(i, k), \quad k \in \mathbb{N}
$$

where

$$
u(i, k)=-\nabla f(x(i, k))+\varepsilon(i, k)-\sum_{j=1, j \neq i}^{N} \alpha_{i j} \nabla_{x(i)} J\left(\left\|x(i, k)-x\left(j_{i}, k\right)\right\|\right) .
$$


Here $x\left(j_{i}, k\right)$ denotes the current solution of thread $j \neq i$ at the time of thread $i$ 's update, and noise term $\varepsilon(i, k)$ comes from one sampling. Thus each $\varepsilon(i, k)$ is i.i.d. with $m$ i.i.d components of mean zero and variance $\sigma^{2}$.

The additional term $-\sum_{j=1, j \neq i}^{N} \alpha_{i j} \nabla_{x_{i}} J\left(\left\|x(i, k)-x\left(j_{i}, k\right)\right\|\right)$ represents the function of mutual attraction and repulsion between individual threads (see Gazi and Passino [31] for reference). $A=\left[\alpha_{i j}\right] \in \mathbb{R}^{N \times N}$ is the coupling matrix with $\alpha_{i j} \in\{0,1\} . \alpha_{i j}=1$ indicates that thread $i$ is informed of the solution identified by threads $j$. We assume that the corresponding graph $\mathcal{G}$ is undirected $\left(A=A^{T}\right)$ and connected.

Denote by $\Delta t(i, k)$ the time needed by thread $i$ to gather one sample for $u(i, k)$, then $x(i, k)$ is obtained at $t(i, k)=\sum_{l<k} \Delta t(i, l)$ in continuous-time. Let $x_{i, t}$ be the solution of thread $i$ at time $t$, satisfying $x_{t(i, k)}=x(i, k)$ and $x_{i, t}:=x_{t(i, k-1)}$ for all $t \in(t(i, k-1), t(i, k))$. Then for each thread $i \in\{1, \ldots, N\}$, the scheme can be written as follows:

$$
x_{i, t(i, k+1)}=x_{i, t(i, k)}+\tilde{\Gamma}\left[-\nabla f\left(x_{i, t(i, k)}\right)+\varepsilon(i, k)-\sum_{j=1, j \neq i}^{N} \alpha_{i j} \nabla_{x_{i}} J\left(\left\|x_{i, t(i, k)}-x_{j, t(i, k)}\right\|\right)\right] .
$$

Define function $g(\cdot)$ as $g(x)=-\nabla_{x} J(\|x\|)$. Let $y_{i, t}=x_{i, t / \tilde{\Gamma}}$ and assume that all $\Delta t(i, k)$ 's are i.i.d with $\mathbb{E}(\Delta t(i, k))=\tilde{\Delta} t$. Similar to (4.4), the dynamics of $y_{i, t}$ can be approximated by

$$
d y_{i, t}=\left[-\nabla f\left(y_{i, t}\right)+\sum_{j=1, j \neq i}^{N} \alpha_{i j} g\left(y_{i, t}-y_{j, t}\right)\right] \tilde{\gamma} d t+\tau \tilde{\gamma} d B_{i, t},
$$

where $\tilde{\gamma}=1 / \tilde{\Delta} t$ and $\tau=\sigma \sqrt{\tilde{\Gamma} \tilde{\Delta} t}$.

Remark 10. In what follows, we shall use the same specification for $g(\cdot)$ as in Chapter 3 (see Gazi and Passino [31]), i.e., $g(\cdot)$ is an odd function of the form:

$$
g(x)=-x\left[g_{a}(\|x\|)-g_{r}(\|x\|)\right],
$$

where $g_{a}: \mathbb{R}^{+} \rightarrow \mathbb{R}^{+}$represents (the magnitude of) the attraction term and it has long range, whereas $g_{r}: \mathbb{R}^{+} \rightarrow \mathbb{R}^{+}$represents (the magnitude of) the repulsion term and it has short range, and $\|\cdot\|$ is the Euclidean norm. We assume that $J(\cdot)$ has a unique minimizer, and there is an equilibrium distance $\rho>0$ such that $g_{a}(\rho)=g_{r}(\rho)$, and for $\|x\|>\rho$ we have 
$g_{a}(\|x\|)>g_{r}(\|x\|)$, and for $\|x\|<\rho$ we have $g_{a}(\|x\|)<g_{r}(\|x\|)$. In this work we consider linear attraction functions, i.e., $g_{a}(\|x\|)=a$ for some $a>0$ and all $\|x\|$, and repulsion functions satisfying $g_{r}(\|x\|)\|x\|^{2} \leq b$ uniformly for some $b>0$.

The choice of parameters $a$ (i.e. attraction) and $b$ (i.e. repulsion) reflects the emphasis on exploration (higher values of $b$ ) versus exploitation (higher values of $a$ ). The potential function is reminiscent of penalty function methods for constrained optimization in which the gradient of the objective function is perturbed so as to ensure updated solutions remain within the feasible region. The difference is that in the flocking approach, potential-induced attraction/repulsion forces keep the updated solutions in a moving ball with fixed size rather than a rigid region. In light of its functionality, the analysis would not change much if we had adopted a different potential function.

\subsection{Analysis}

In this section we study the stochastic processes $\left\{y_{i, t}: t \geq 0\right\}$ associated with each one of the $N>1$ threads in the flocking-based approach. The average solution $\bar{y}_{t}=(1 / N) \sum_{i=1}^{N} y_{i, t}$ will be of particular importance in characterizing the performance of the flocking approach. The first part of the analysis pertains to a characterization of cohesiveness of the solutions identified by the different threads. To this end, we will analyze the process $\left\{\bar{V}_{t}: t>0\right\}$ defined as

$$
\bar{V}_{t}=\frac{1}{N} \sum_{i=1}^{N} \frac{1}{2}\left\|y_{i, t}-\bar{y}_{t}\right\|^{2} .
$$

\subsubsection{Preliminaries}

We consider the stochastic differential equation governing $\bar{V}_{t}$. Let $V_{i, t}=(1 / 2)\left\|e_{i, t}\right\|^{2}$ with $e_{i, t}=y_{i, t}-\bar{y}_{t}$. We have $\bar{V}_{t}=(1 / N) \sum_{i=1}^{N} V_{i, t}$. Applying Ito's formula,

$$
d V_{i, t}=d e_{i, t} \cdot e_{i, t}+\frac{1}{2} d e_{i, t} \cdot d e_{i, t}
$$

where $d e_{i, t}=d y_{i, t}-d \bar{y}_{t}$. 


\section{Lemma 1.}

$$
\begin{aligned}
& d \bar{V}_{t} \\
& =-\frac{a}{N} \sum_{i=1}^{N} \sum_{j=1, j \neq i}^{N} \alpha_{i j}\left(e_{i, t}-e_{j, t}\right)^{T} e_{i, t} \tilde{\gamma} d t+\frac{1}{2 N} \sum_{i=1}^{N} \sum_{j=1, j \neq i}^{N} \alpha_{i j} g_{r}\left(\left\|y_{i, t}-x_{j, t}\right\|\right)\left\|y_{i, t}-y_{j, t}\right\|^{2} \tilde{\gamma} d t \\
& \quad-\frac{1}{N} \sum_{i=1}^{N} \nabla^{T} f\left(y_{i, t}\right) e_{i, t} \tilde{\gamma} d t+\frac{\tau}{N} \tilde{\gamma} \sum_{i=1}^{N} d B_{i, t}^{T} e_{i, t}+\frac{m \tau^{2} \tilde{\gamma}^{2}(N-1)}{2 N} d t .
\end{aligned}
$$

Proof. See Appendix 4.7.1.

\subsubsection{Cohesiveness}

Let $L=\left[l_{i j}\right]$ be the Laplacian matrix associated with the adjacency matrix $A$. Notice that

$$
-\sum_{i=1}^{N} \sum_{j=1, j \neq i}^{N} \alpha_{i j}\left(e_{i, t}-e_{j, t}\right)^{T} e_{i, t}=\sum_{i=1}^{N} \sum_{j=1}^{N} l_{i j}\left(e_{i, t}-e_{j, t}\right)^{T} e_{i, t}=\sum_{i=1}^{N} \sum_{j=1}^{N} l_{i j} e_{j, t}^{T} e_{i, t} .
$$

Let $\mathbf{e}_{t}=\left[e_{1, t}^{T}, \ldots, e_{N, t}^{T}\right]^{T}$. Since graph $\mathcal{G}$ is connected, $\lambda_{2}(L)>0$ (see Godsil and Royle [36]) and

$$
\sum_{i=1}^{N} \sum_{j=1}^{N} l_{i j} e_{j, t}^{T} e_{i, t}=\mathbf{e}^{T}\left(L \otimes I_{m}\right) \mathbf{e} \geq \lambda_{2} \mathbf{e}^{T} \mathbf{e}=\lambda_{2} \sum_{i=1}^{N}\left\|e_{i}\right\|^{2}
$$

where $\lambda_{2}:=\lambda_{2}(L)$ is the second-smallest eigenvalue of $L$, or the algebraic connectivity of $\mathcal{G}$.

The following result provides a characterization of degree of cohesiveness of sample paths associated with different individual threads.

Theorem 3. Assume there exists $\eta>0$ such that $\|\nabla f(x)\| \leq \eta$ for all $x$. Then the ensemble average of $\bar{V}_{t}$ is uniformly bounded in $t$ :

$$
\mathbb{E}\left[\bar{V}_{t}\right] \leq e^{-c_{1} \tilde{\gamma} t} \bar{V}_{0}+\frac{c_{2}}{c_{1}}\left(1-e^{-c_{1} \tilde{\gamma} t}\right)
$$

where $c_{1} \in\left(0,2 a \lambda_{2}\right)$ is arbitrary, and

$$
c_{2}=\frac{\eta^{2}}{2\left(2 a \lambda_{2}-c_{1}\right)}+\frac{b|\operatorname{Tr}(L)|}{2 N}+\frac{m \tau^{2}(N-1)}{2 N} \tilde{\gamma}
$$


In particular,

$$
\mathbb{E}\left[\bar{V}_{t}\right] \leq \max \left\{\bar{V}_{0}, \psi^{*}(N)\right\},
$$

and in the long run, $\mathbb{E}\left[\bar{V}_{t}\right] \leq \psi^{*}(N)$ where

$$
\psi^{*}(N)=\frac{1}{2 a \lambda_{2}}\left[\sqrt{\frac{\eta^{2}}{4 a \lambda_{2}}+\frac{b|\operatorname{Tr}(L)|}{2 N}+\frac{m \tau^{2}(N-1)}{2 N} \tilde{\gamma}}+\sqrt{\frac{\eta^{2}}{4 a \lambda_{2}}}\right]^{2} .
$$

Proof. See Appendix 4.7.2.

Remark 11. Note that the upper bound on the ensemble average of $\bar{V}_{t}$ is decreasing in $a$ (attraction potential) and increasing in $b$ (repulsive potential). Hence, the relative strength of these parameters implies a trade-off between exploration (less cohesive solutions) and exploitation (more cohesive solutions).

Remark 12. Note further that the algebraic connectivity $\lambda_{2}$ is critical in determining the upper bound of $\bar{V}_{t}$. When $N$ is fixed, a larger $\lambda_{2}$ leads to a smaller upper bound. In a complete graph, $\lambda_{2}$ achieves its maximum value $N$. In this case,

$$
\lim _{N \rightarrow \infty} \psi^{*}(N)=\lim _{N \rightarrow \infty} \frac{1}{2 a N}\left[\sqrt{\frac{\eta^{2}}{4 a N}+\frac{b(N-1)}{2}+\frac{m \tau^{2}(N-1)}{2 N}} \tilde{\gamma}+\sqrt{\frac{\eta^{2}}{4 a N}}\right]^{2}=\frac{b}{4 a} .
$$

With a large number of threads, cohesiveness is ensured by the choice of $b / a$ governing the interplay between inter-individual attraction and repulsion.

\subsection{Noise Reduction in Convex Optimization}

In this section we formalize the noise reduction properties of the flocking-based algorithmic scheme for convex optimization. Repulsion amongst threads prevents duplication of search effort which may arise for instance, when there are multiple locally optimal solutions. Thus, for convex optimization problems, there is no need for a "repulsion" amongst individual threads and in this section we set $b=0$. As we shall see below, when the underlying problem 
is not convex, repulsion amongst threads does facilitate the identification of a globally optimal solution.

We make the following standing assumptions:

Assumption 4. (Strong convexity) $\left(\nabla f(x)-\nabla f\left(x^{\prime}\right)\right)^{T}\left(x-x^{\prime}\right) \geq \kappa\left\|x-x^{\prime}\right\|^{2}$ for some $\kappa>0$ and for all $x, x^{\prime}$.

Assumption 5. (Lipschitz) $\left\|\nabla f(x)-\nabla f\left(x^{\prime}\right)\right\| \leq \mu\left\|x-x^{\prime}\right\|$ for some $\mu \geq \kappa$ and for all $x \neq x^{\prime}$.

Since $b=0$, Equation (4.8) can be simplified to

$$
\begin{array}{r}
d \bar{V}_{t}=-\frac{a}{N} \sum_{i=1}^{N} \sum_{j=1, j \neq i}^{N} \alpha_{i j}\left(e_{i, t}-e_{j, t}\right)^{T} e_{i} \tilde{\gamma} d t-\frac{1}{N} \sum_{i=1}^{N} \nabla^{T} f\left(y_{i, t}\right) e_{i, t} \tilde{\gamma} d t+\frac{\tau}{N} \tilde{\gamma} \sum_{i=1}^{N} d B_{i, t}^{T} e_{i, t} \\
+\frac{m \tau^{2} \tilde{\gamma}^{2}(N-1)}{2 N} d t
\end{array}
$$

Let us introduce a measure $U_{t}=(1 / 2)\left\|\bar{y}_{t}-x^{*}\right\|^{2}$, of the distance between the average solution identified by all threads at time $t / \Gamma$ and the unique optimal solution $x^{*}$. Let $F_{i, t}=(1 / 2)\left\|y_{i, t}-x^{*}\right\|^{2}$ and $\bar{F}_{t}=(1 / N) \sum_{i=1}^{N} F_{i, t}$. Notice that

$$
\begin{aligned}
\bar{F}_{t} & =\frac{1}{N} \sum_{i=1}^{N} \frac{1}{2}\left(y_{i, t}-\bar{y}_{t}+\bar{y}_{t}-x^{*}\right) \cdot\left(y_{i, t}-\bar{y}_{t}+\bar{y}_{t}-x^{*}\right) \\
& =\frac{1}{N} \sum_{i=1}^{N} \frac{1}{2}\left\|y_{i, t}-\bar{y}_{t}\right\|^{2}+\frac{1}{N} \sum_{i=1}^{N} \frac{1}{2}\left\|\bar{y}_{t}-x^{*}\right\|^{2}+\frac{1}{N} \sum_{i=1}^{N}\left(y_{i, t}-\bar{y}_{t}\right) \cdot\left(\bar{y}_{t}-x^{*}\right) \\
& =\bar{V}_{t}+U_{t} .
\end{aligned}
$$

The following result provides a characterization of the performance of the flocking-based approach to stochastic optimization under Assumptions 1 and 2.

Theorem 4. Suppose that Assumptions 1 and 2 hold. The ensemble average of $U_{t}$ is uniformly bounded:

$$
\begin{aligned}
\mathbb{E}\left[U_{t}\right] & =\frac{1}{2} \mathbb{E}\left[\left\|\bar{y}_{t}-x^{*}\right\|^{2}\right] \\
& \leq e^{-2 \kappa \tilde{\gamma} t}\left[U_{0}+\frac{(\mu-\kappa)}{a \lambda_{2}} \bar{V}_{0}\right]+\frac{m \tau^{2} \tilde{\gamma}}{4 \kappa N}\left[1+\frac{(\mu-\kappa)(N-1)}{a \lambda_{2}}\right]\left(1-e^{-2 \kappa \tilde{\gamma} t}\right) .
\end{aligned}
$$


In the long-run the upper bound is:

$$
\limsup _{t \rightarrow \infty} \frac{1}{2} \mathbb{E}\left[\left\|\bar{y}_{t}-x^{*}\right\|^{2}\right] \leq \phi^{*}(N)=\frac{m \tau^{2} \tilde{\gamma}}{4 \kappa N}\left[1+\frac{(\mu-\kappa)(N-1)}{a \lambda_{2}}\right] .
$$

Proof. See Appendix 4.7.3.

Corollary 2. Let $\bar{x}_{t}=(1 / N) \sum_{i=1}^{N} x_{i, t}$. We have

$$
\frac{1}{2} \mathbb{E}\left[\left\|\bar{x}_{t}-x^{*}\right\|^{2}\right] \leq e^{-2 \kappa \tilde{\gamma} \tilde{\Gamma} t}\left[U_{0}+\frac{(\mu-\kappa)}{a \lambda_{2}} \bar{V}_{0}\right]+\frac{m \tau^{2} \tilde{\gamma}}{4 \kappa N}\left[1+\frac{(\mu-\kappa)(N-1)}{a \lambda_{2}}\right]\left(1-e^{-2 \kappa \tilde{\gamma} \tilde{\Gamma} t}\right) .
$$

Proof. Since $y_{i, t}=x_{i, t / \tilde{\Gamma}}, \mathbb{E}\left[\left\|\bar{x}_{t}-x^{*}\right\|^{2}\right]=\mathbb{E}\left[U_{\tilde{\Gamma} t}\right]$. The above result follows immediately from Theorem 4.

Note that if we choose $a$ such that $a \lambda_{2} \sim N, \phi^{*}(N) \sim 1 / N$, then the long-run upper bound of $\mathbb{E}\left[\left\|\bar{x}_{t}-x^{*}\right\|^{2}\right]$ is monotonically decreasing in $N$. In what follows, we will show that the flocking-based approach exhibits a noise reduction property that is similar to that of a stochastic gradient algorithm based upon the average of $N$ gradient samples.

Let us assume there is no time overhead in the centralized algorithm so that $\Delta t=\tilde{\Delta} t$. Also assume $\Gamma=\tilde{\Gamma}$. It follows that $\gamma=\tilde{\gamma}, \tau_{N}=\sigma \sqrt{\Gamma /(\gamma N)}$ and $\tau=\sigma \sqrt{\Gamma / \gamma}$.

The stochastic differential equation for the algorithm based upon the average of $N$ gradient samples is:

$$
d y_{t}=-\nabla f\left(y_{t}\right) \gamma d t+\tau_{N} \gamma d B_{t} .
$$

Let $G_{t}=(1 / 2)\left\|y_{t}-x^{*}\right\|^{2}$. It follows that

$$
d G_{t}=d y_{t} \cdot y_{t}+\frac{1}{2} d y_{t} \cdot d y_{t}=-\nabla^{T} f\left(y_{t}\right) y_{t} \gamma d t+\tau_{N} \gamma d B_{t}^{T} y_{t}+\frac{1}{2} m \tau_{N}^{2} \gamma^{2} d t .
$$

Then,

$$
d G_{t} \leq-2 \kappa \gamma G_{t} d t+\frac{1}{2} m \tau_{N}^{2} \gamma^{2} d t+\tau_{N} \gamma d B_{t}^{T} y_{t} .
$$

As in the proof of Theorem 2, it can be shown that

$$
\mathbb{E}\left[G_{t}\right] \leq e^{-2 \kappa \gamma t} G_{0}+\frac{m \tau_{N}^{2} \gamma}{4 \kappa}\left(1-e^{-2 \kappa \gamma t}\right)=e^{-2 \kappa \gamma t} G_{0}+\frac{m \sigma^{2} \Gamma}{4 \kappa N}\left(1-e^{-2 \kappa \gamma t}\right) .
$$


Therefore,

$$
\frac{1}{2} \mathbb{E}\left[\left\|x_{t}-x^{*}\right\|^{2}\right]=\mathbb{E}\left[G_{\Gamma t}\right] \leq e^{-2 \kappa \gamma \Gamma t} G_{0}+\frac{m \sigma^{2} \Gamma}{4 \kappa N}\left(1-e^{-2 \kappa \gamma \Gamma t}\right) .
$$

In the long run,

$$
\limsup _{t \rightarrow \infty} \frac{1}{2} \mathbb{E}\left[\left\|x_{t}-x^{*}\right\|^{2}\right] \leq \frac{m \sigma^{2} \Gamma}{4 \kappa N}
$$

Since $\tau=\sigma \sqrt{\Gamma / \gamma}$, a comparison with the upper bound obtained in Corollary 2 (when $\left.a \lambda_{2} \sim N\right)$ readily indicates that the flocking-based approach exhibits a noise reduction property that is similar to that of a stochastic gradient algorithm based upon the average of $N$ gradient samples. In the next section, we show that the flocking-based approach outperforms (in real-time) stochastic gradient algorithm based upon the average of $N$ gradient samples when overhead due to synchronization is taken into account.

\subsubsection{Real-time Performance Comparison}

When sampling is undertaken in parallel, synchronization is needed to execute the tasks that can not be executed in parallel. Hence, the improvement obtained by parallel sampling and centralized gradient estimation is limited by overhead related to (i) time spent gathering samples and (ii) synchronization. See for example Hill and Marty [40] for a discussion on Amdahl's law in multi-core processing. In what follows we will account for overhead by assuming the total time needed to implement an iteration of stochastic gradient algorithm with $N$ samples obtained in parallel is monotonically increasing in $N$, i.e., $\Delta t \sim N^{1 / \beta} \tilde{\Delta} t$ where $\beta>1$. The parameter $\beta>1$ encapsulates the relative burden of overhead so that when $\beta \gg 1$, the burden is relatively weak but increases with values closer to 1 .

In a way similar to the analysis presented in the previous section, we get

$$
d G_{t} \geq-2 \mu \gamma G_{t} d t+\frac{1}{2} m \tau_{N}^{2} \gamma^{2} d t+\tau_{N} \gamma d B_{t}^{T} y_{t} .
$$

A lower bound could then be obtained:

$$
\mathbb{E}\left[G_{t}\right] \geq e^{-2 \mu \gamma t} G_{0}+\frac{m \tau_{N}^{2} \gamma}{4 \mu}\left(1-e^{-2 \mu \gamma t}\right) .
$$


Then,

$$
\frac{1}{2} \mathbb{E}\left[\left\|x_{t}-x^{*}\right\|^{2}\right]=\mathbb{E}\left[G_{\Gamma t}\right] \geq e^{-2 \mu \gamma \Gamma t} G_{0}+\frac{m \tau_{N}^{2} \gamma}{4 \mu}\left(1-e^{-2 \mu \gamma \Gamma t}\right),
$$

and in the long run,

$$
\frac{1}{2} \mathbb{E}\left[\left\|x_{t}-x^{*}\right\|^{2}\right] \geq \frac{m \tau_{N}^{2} \gamma}{4 \mu}=\frac{m \sigma^{2} \Gamma}{4 \mu N} .
$$

In what follows we consider two specific scenarios.

In the case that the two algorithms use the same step size $\Gamma=\tilde{\Gamma}$, recalling that $\gamma=1 / \Delta t$ and $\tilde{\gamma}=1 / \tilde{\Delta} t$, we have $\gamma=(\tilde{\Delta} t / \Delta t) \tilde{\gamma} \sim N^{-1 / \beta} \tilde{\gamma}$. It follows that $2 \mu \gamma \Gamma /(2 \kappa \tilde{\gamma} \tilde{\Gamma}) \sim N^{-1 / \beta}$. Hence the convergence speed of the centralized scheme is slower than that of a flocking-based scheme. The long-run performance of the centralized implementation is bounded below by $m \sigma^{2} \Gamma /(4 \mu N) \sim 1 / N$, which is on the same level of the flocking-based algorithm.

In the case that the two algorithms use stepsizes proportional to the average sampling times, i.e., $\Gamma / \Delta t=\tilde{\Gamma} / \tilde{\Delta} t, \mathbb{E}\left[\left\|\bar{x}_{t}-x^{*}\right\|^{2}\right]$ and $\mathbb{E}\left[\left\|x_{t}-x^{*}\right\|^{2}\right]$ have the same convergence speed since

$$
2 \mu \tilde{\gamma} \tilde{\Gamma}=2 \mu \tilde{\Gamma} / \tilde{\Delta} t=2 \mu \Gamma / \Delta t=2 \mu \gamma \Gamma
$$

However,

$$
\frac{m \tau_{N}^{2} \gamma}{4 \mu}=\frac{m \sigma^{2} \Gamma}{4 \mu N}=\frac{m \sigma^{2} \tilde{\Gamma}}{4 \mu N} \frac{\Delta t}{\tilde{\Delta} t} \sim N^{1 / \beta-1} .
$$

We formalize the claim that the flocking approach (each thread with a single gradient sample) outperforms the stochastic gradient approach based upon $N$ samples per step in finite time.

Proposition 1. Suppose $\Delta t=N^{1 / \beta} \tilde{\Delta} t$ for some $\beta>0$ and $\Gamma / \Delta t=\tilde{\Gamma} / \tilde{\Delta} t$. Then there exists $N^{*}<\infty$ and $t^{*}<\infty$ such

$$
\mathbb{E}\left[\left\|x_{t}-x^{*}\right\|^{2}\right]>\mathbb{E}\left[\left\|\bar{x}_{t}-x^{*}\right\|^{2}\right]
$$

for all $N>N^{*}$ and $t>t^{*}$.

Proof. See Appendix 4.7.4. 


\subsection{Application to Non-Convex Optimization}

In this section, we apply the flocking-based algorithm to the optimization of general nonconvex functions. We will provide some motivating simulation examples first and then discuss the global asymptotic properties of this scheme.

\subsubsection{Simulation Examples}

In this part, we illustrate with a limited simulation testbed the performance benefits of a flocking-based approach when the objective function is not convex. The results indicate that the noise reduction property is maintained. They also suggest that a flocking-based approach seems better suited to escape locally optimal solutions than a stochastic gradient descent based upon the average of $N$ samples. This is likely due to the repulsive force which enforces a certain level of diversity in the set of candidate solutions. The flocking based-gradient descent dynamics are thus more likely to lead to globally optimal solutions. We assume that $\Gamma / \Delta t=\tilde{\Gamma} / \tilde{\Delta} t$ in the following simulation examples.

\section{Ackley's Function (Case 1)}

We first consider Ackley's function

$$
f(x, y)=-20 \exp \left(-0.2 \sqrt{0.5\left(x^{2}+y^{2}\right)}\right)-\exp (0.5(\cos (2 \pi x)+\cos (2 \pi y)))+e+20
$$

It has a global minimum at $x^{*}=(0,0)$ and various local optima.

We use $N=20$ parallel threads, and the attraction/repulsion function $g(x)=-x[4-$ $\left.800 \exp \left(-\|x\|^{2}\right)\right]$ is adopted. In the flocking-based approach, each thread is randomly connected with 8 other threads. Overhead parameter $\beta=5$. Sampling times are constant with $\tilde{\Delta} t=1$ and $\Delta t=N^{1 / \beta} \tilde{\Delta} t \simeq 1.82$, respectively. Noise level is $\sigma=0.5$. Initially, all the sampling points were distributed randomly in the $[10,15] \times[10,15]$ interval. Simulations run 10 times (60s each). Performances of the centralized algorithm are shown in Figure 4.2(a). It 

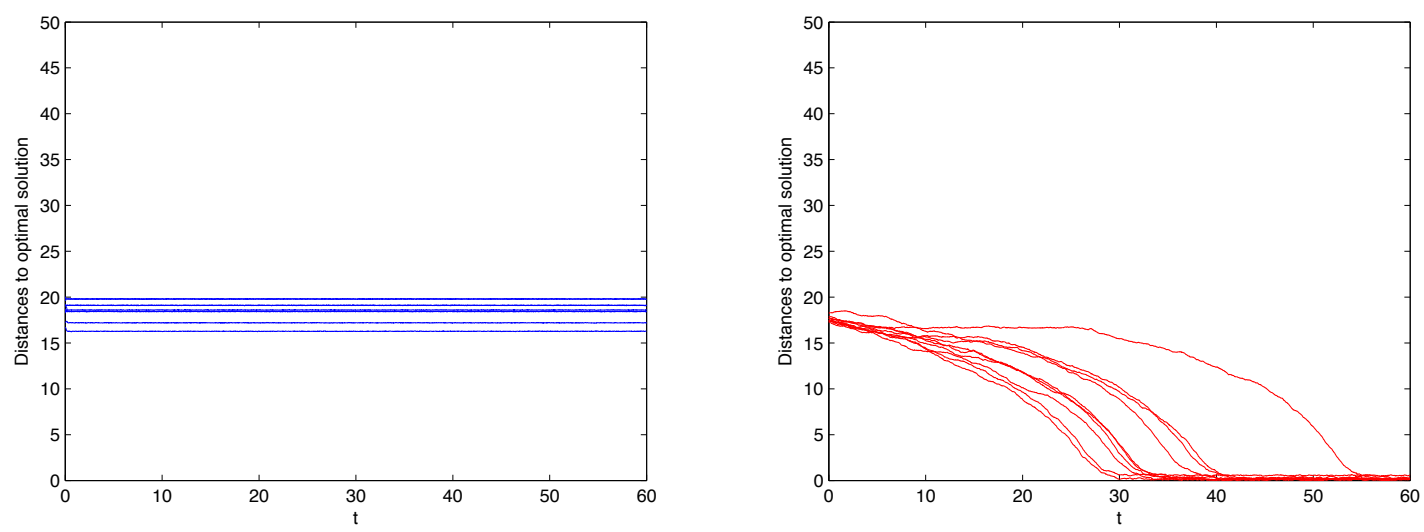

(a) Distance to optimum for sample average (b) Distance to optimum with flocking discipline. scheme with $N$ samples.

Figure 4.2: Performance comparison for Ackley's function (Case 1).

is clear that all independent threads got trapped in local optima. By contrast, we can see in Figure 4.2 that $\bar{x}_{t}$ in the flocking-based scheme approaches the global optimum successfully. This is likely due to the repulsive force which enforces a certain level of diversity in the set of candidate solutions.

\section{Ackley's Function (Case 2)}

In this case we assume that the noise level is $\sigma=7$. We use $N=30$ computing threads, and the attraction/repulsion function $g(x)=-x\left[3-0.01 \exp \left(-\|x\|^{2}\right)\right]$ is adopted. In the flockingbased approach, each thread randomly communicates with 8 other threads. Overhead parameter $\beta=1.5$. Sampling times are constant with $\tilde{\Delta} t=0.02$ and $\Delta t=N^{1 / \beta} \tilde{\Delta} t \simeq 0.092$, respectively. Initially, the sampling points were distributed randomly in the $[10,12] \times[10,12]$ interval. Simulations run for 36s.

We can see in Figure 4.3(a) that individual threads operating in parallel are not able to approach the globally optimal solution. On the contrary, the flocking discipline allows convergence to the global optimum successfully (see Figure 4.3(b)). 

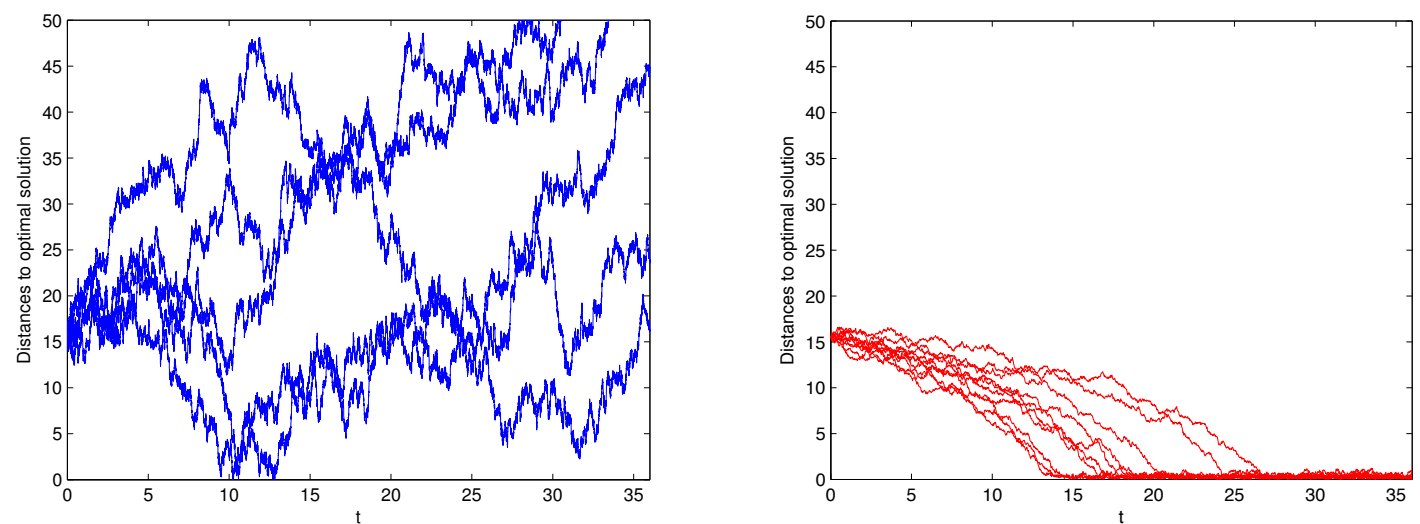

(a) Distance to optimum for sample average (b) Distance to optimum with flocking discipline. scheme with $N$ samples.

Figure 4.3: Performance comparison for Ackley's function (Case 2).

\subsubsection{Asymptotic Noise Reduction}

We now discuss the asymptotic noise reduction properties of the flocking-based algorithmic scheme for non-convex optimization. We start by reviewing the asymptotic performance of the centralized algorithm based upon the average of $N$ samples per step. Recalling (4.4),

$$
d y_{t}=-\nabla f\left(y_{t}\right) \gamma d t+\tau_{N} \gamma d B_{t},
$$

The limiting density of $y_{t}$ (which solves a related Fokker-Planck equation (see $\left.[28,81]\right)$ ) is

$$
\hat{\pi}\left(y_{t}\right)=\frac{\exp \left\{-2 f(x) /\left(\tau_{N}^{2} \gamma\right)\right\}}{\int \exp \left\{-2 f(y) /\left(\tau_{N}^{2} \gamma\right)\right\}}=\frac{\exp \left\{-2 N f(y) /\left(\sigma^{2} \tilde{\Gamma}\right)\right\}}{\int \exp \left\{-2 N f(y) /\left(\sigma^{2} \tilde{\Gamma}\right)\right\}}
$$

assuming that $\Gamma=\tilde{\Gamma}$.

We return now to the flocking scheme. By Equation (4.6) and the relation $g(x)=$ $-\nabla_{x} J(\|x\|)$, we have

$$
d y_{i, t}=\left[-\nabla f\left(y_{i, t}\right)-\sum_{j=1, j \neq i}^{N} \alpha_{i j} \nabla_{x_{i}} J\left\|y_{i, t}-y_{j, t}\right\|\right] \tilde{\gamma} d t+\tau \tilde{\gamma} d B_{i, t} .
$$

Let $\mathbf{y}_{t}=\left[y_{1, t}^{T}, \ldots, y_{N, t}^{T}\right]^{T} \in \mathbb{R}^{N \times m}, \mathbf{B}_{\mathbf{t}}=\left[B_{1, t}^{T}, \ldots, B_{N, t}^{T}\right]^{T} \in \mathbb{R}^{N \times m}$. Define

$$
H\left(\mathbf{y}_{t}\right)=\sum_{i=1}^{N} f\left(y_{i, t}\right)+\frac{1}{2} \sum_{i=1}^{N} \sum_{j=1, j \neq i}^{N} \alpha_{i j} J\left(\left\|y_{i, t}-y_{j, t}\right\|\right) .
$$


We can rewrite (4.21) in a compact form:

$$
d \mathbf{y}_{t}=-\nabla H\left(\mathbf{y}_{t}\right) \tilde{\gamma} d t+\tau \tilde{\gamma} d \mathbf{B}_{t}
$$

The Fokker-Planck equation related to the stochastic differential equation (4.23) is:

$$
\frac{\partial p_{t}}{\partial t}=-\nabla \cdot\left(\nabla H(\mathbf{y}) \tilde{\gamma} p_{t}\right)+\frac{\tau^{2} \tilde{\gamma}^{2}}{2} \nabla^{2} p_{t}
$$

where $p_{t}:=p\left(\mathbf{y}_{t}, t \mid \mathbf{y}_{0}, 0\right)$ is the probability density of $\mathbf{y}_{t}$.

Proposition 2. Assume that $\int \exp \left\{-2 H(\mathbf{y}) / \tau^{2}\right\} d \mathbf{y}$ is finite. Then $\mathbf{y}_{t}$ weakly approaches a unique equilibrium, which is a Gibbs distribution with density

$$
\pi(\mathbf{y})=\frac{1}{K} \exp \left\{-2 H(\mathbf{y}) /\left(\tau^{2} \tilde{\gamma}\right)\right\}
$$

where

$$
K=\int \exp \left\{-2 H(\mathbf{y}) /\left(\tau^{2} \tilde{\gamma}\right)\right\} d \mathbf{y}
$$

Proposition 2 comes from the standard theory of diffusion (see $[28,81]$ ). The assumption is required for the distribution (4.25) to be well-defined. It is satisfied when $H(\mathbf{y})$ grows rapidly enough, or when the feasible region $X$ has reflected boundaries.

We now show that for large values of the parameter $a$ (attractive force) the asymptotic noise reduction properties of the flocking-based algorithmic scheme is the same to that of a centralized algorithm based upon the average of $N$ samples per step.

Theorem 5. The asymptotic probability distribution of $\bar{y}_{t}$ has density:

$$
\pi^{*}(\bar{y})=\frac{\exp \left\{-2 N f(\bar{y}) /\left(\sigma^{2} \tilde{\Gamma}\right)\right\}}{\int \exp \left\{-2 N f(\bar{y}) /\left(\sigma^{2} \tilde{\Gamma}\right)\right\} d \bar{y}},
$$

as $a \rightarrow \infty$.

Proof. See Appendix 4.7.5.

Remark 13. The asymptotic probability distribution of $\bar{x}_{t}$ is the same as that of $\bar{y}_{t}$ as $a \rightarrow \infty$. 
Remark 14 . The limiting probability density of $\bar{x}_{t}$ as $a \rightarrow \infty$ does not depend on the specific network topology as long as $\lambda_{2}>0$, i.e., the network is connected. This is a very mild networking requirement satisfied by many simple topologies (e.g. ring, line, bus, mesh).

Remark 15. As $N \rightarrow \infty, \pi^{*}(\bar{x})$ concentrates on the global minima of $f$, in which case the average solution of the flocking scheme is guaranteed to approximate a global minimum (see Geman and Hwang [34] for a reference).

\subsection{Conclusions of This Chapter}

In recent years, the paradigm of cloud computing has emerged as an architecture for computing that makes use of distributed (networked) computing resources. In this chapter, we analyze a distributed computing algorithmic scheme for stochastic optimization which relies on modest communication requirements amongst processors and most importantly, does not require synchronization. The proposed distributed algorithmic framework may provide significant speed-up in application domains in which sampling times are non-negligible. This is the case, for example, in the optimization of complex systems for which performance may only be evaluated via computationally intensive black-box simulation models.

The scheme considered in this chapter has $N>1$ computing threads operating under a connected network. At each step, each thread independently computes a new solution by using a noisy estimation of the gradient, which is further perturbed by a combination of repulsive and attractive terms depending upon the relative distance to solutions identified by neighboring threads. When the objective function is convex, we showed that a flocking-like approach for distributed stochastic optimization provides a noise reduction effect similar to that of a centralized stochastic gradient algorithm based upon the average of $N$ gradient samples at each step. When the overhead related to the time needed to gather $N$ samples and synchronization is not negligible, the flocking implementation outperforms a centralized stochastic gradient algorithm based upon the average of $N$ gradient samples at each step. When the objective function is not convex, the flocking-based approach seems better suited to escape locally optimal solutions due to the repulsive force which enforces a certain level 
of diversity in the set of candidate solutions. Here again, we showed that the noise reduction effect is similar to that associated to the centralized stochastic gradient algorithm based upon the average of $N$ gradient samples at each step.

\subsection{Appendix}

\subsubsection{Proof of Lemma 1}

Since $A=A^{T}$ and $g(\cdot)$ is odd,

$$
\begin{aligned}
d \bar{y}_{t} & =\frac{1}{N} \sum_{i=1}^{N} d y_{i, t} \\
& =\frac{1}{N} \sum_{i=1}^{N}\left[-\nabla f\left(y_{i, t}\right) \tilde{\gamma} d t+\sum_{j=1, j \neq i}^{N} \alpha_{i j} g\left(y_{i, t}-y_{j, t}\right) \tilde{\gamma} d t+\tau \tilde{\gamma} d B_{i, t}\right] \\
& =-\frac{1}{N} \sum_{i=1}^{N} \nabla f\left(y_{i, t}\right) \tilde{\gamma} d t+\frac{\tau}{N} \tilde{\gamma} \sum_{i=1}^{N} d B_{i, t} .
\end{aligned}
$$

It follows that

$$
\begin{aligned}
d e_{i, t}= & d y_{i, t}-d \bar{y}_{t} \\
= & \sum_{j=1, j \neq i}^{N} \alpha_{i j} g\left(y_{i, t}-y_{j, t}\right) \tilde{\gamma} d t-\nabla f\left(y_{i, t}\right) \tilde{\gamma} d t+\frac{1}{N} \sum_{i=1}^{N} \nabla f\left(x_{i, t}\right) \tilde{\gamma} d t+\tau \tilde{\gamma} d B_{i, t} \\
& -\frac{\tau}{N} \tilde{\gamma} \sum_{i=1}^{N} d B_{i, t} .
\end{aligned}
$$

From Equation (4.7),

$$
\begin{aligned}
g\left(y_{i, t}-y_{j, t}\right) & =-\left(y_{i, t}-y_{j, t}\right)\left[g_{a}\left(\left\|y_{i, t}-y_{j, t}\right\|\right)-g_{r}\left(\left\|y_{i, t}-y_{j, t}\right\|\right)\right] \\
& =-\left(y_{i, t}-y_{j, t}\right)\left[a-g_{r}\left(\left\|y_{i, t}-y_{j, t}\right\|\right)\right] .
\end{aligned}
$$

We then have

$$
\sum_{j=1, j \neq i}^{N} \alpha_{i j} g\left(y_{i, t}-y_{j, t}\right)=-a \sum_{j=1, j \neq i}^{N} \alpha_{i j}\left(y_{i, t}-y_{j, t}\right)+\sum_{j=1, j \neq i}^{N} \alpha_{i j} g_{r}\left(\left\|y_{i, t}-y_{j, t}\right\|\right)\left(y_{i, t}-y_{j, t}\right) .
$$


Consequently, Equation (4.27) becomes

$$
\begin{array}{r}
d e_{i, t}=-a \sum_{j=1, j \neq i}^{N} \alpha_{i j}\left(y_{i, t}-y_{j, t}\right) \tilde{\gamma} d t+\sum_{j=1, j \neq i}^{N} \alpha_{i j} g_{r}\left(\left\|y_{i, t}-y_{j, t}\right\|\right)\left(y_{i, t}-y_{j, t}\right) \tilde{\gamma} d t \\
\quad-\nabla f\left(y_{i, t}\right) \tilde{\gamma} d t+\frac{1}{N} \sum_{j=1}^{N} \nabla f\left(x_{j, t}\right) \tilde{\gamma} d t+\tau \tilde{\gamma} d B_{i, t}-\frac{\tau}{N} \tilde{\gamma} \sum_{j=1}^{N} d B_{j, t} .
\end{array}
$$

Then,

$$
\begin{aligned}
d V_{i, t}= & d e_{i, t} \cdot e_{i, t}+\frac{1}{2} d e_{i, t} \cdot d e_{i, t} \\
= & -a \sum_{j=1, j \neq i}^{N} \alpha_{i j}\left(y_{i, t}-y_{j, t}\right)^{T} e_{i, t} \tilde{\gamma} d t+\sum_{j=1, j \neq i}^{N} \alpha_{i j} g_{r}\left(\left\|y_{i, t}-y_{j, t}\right\|\right)\left(y_{i, t}-y_{j, t}\right)^{T} e_{i, t} \tilde{\gamma} d t \\
& -\left[\nabla f\left(y_{i, t}\right)-\frac{1}{N} \sum_{j=1}^{N} \nabla f\left(x_{j, t}\right)\right]^{T} e_{i, t} \tilde{\gamma} d t+\left[\tau \tilde{\gamma} d B_{i, t}-\frac{\tau}{N} \tilde{\gamma} \sum_{j=1}^{N} d B_{j, t}\right]^{T} e_{i, t} \\
& +\frac{m \tau^{2} \tilde{\gamma}^{2}}{2}\left(1-\frac{1}{N}\right) d t .
\end{aligned}
$$

In conclusion, the stochastic differential equation is

$$
\begin{aligned}
d \bar{V}_{t}= & \frac{1}{N} \sum_{i=1}^{N} d V_{i, t} \\
= & -\frac{a}{N} \sum_{i=1}^{N} \sum_{j=1, j \neq i}^{N} \alpha_{i j}\left(y_{i, t}-y_{j, t}\right)^{T} e_{i, t} \tilde{\gamma} d t \\
& +\frac{1}{N} \sum_{i=1}^{N} \sum_{j=1, j \neq i}^{N} \alpha_{i j} g_{r}\left(\left\|y_{i, t}-y_{j, t}\right\|\right)\left(y_{i, t}-y_{j, t}\right)^{T} e_{i, t} \tilde{\gamma} d t-\frac{1}{N} \sum_{i=1}^{N} \nabla^{T} f\left(y_{i, t}\right) e_{i, t} \tilde{\gamma} d t \\
& +\frac{\tau}{N} \tilde{\gamma} \sum_{i=1}^{N} d B_{i, t}^{T} e_{i, t}+\frac{m \tau^{2} \tilde{\gamma}^{2}(N-1)}{2 N} d t \\
= & -\frac{a}{N} \sum_{i=1}^{N} \sum_{j=1, j \neq i}^{N} \alpha_{i j}\left(e_{i, t}-e_{j, t}\right)^{T} e_{i, t} \tilde{\gamma} d t \\
& +\frac{1}{2 N} \sum_{i=1}^{N} \sum_{j=1, j \neq i}^{N} \alpha_{i j} g_{r}\left(\left\|y_{i, t}-y_{j, t}\right\|\right)\left\|y_{i, t}-y_{j, t}\right\|^{2} \tilde{\gamma} d t-\frac{1}{N} \sum_{i=1}^{N} \nabla^{T} f\left(y_{i, t}\right) e_{i, t} \tilde{\gamma} d t \\
& +\frac{\tau}{N} \tilde{\gamma} \sum_{i=1}^{N} d B_{i, t}^{T} e_{i, t}+\frac{m \tau^{2} \tilde{\gamma}^{2}(N-1)}{2 N} d t .
\end{aligned}
$$




\subsubsection{Proof of Theorem 3}

By Equation (4.30),

$$
\begin{aligned}
d \bar{V}_{t}=-\frac{a}{N} \sum_{i=1}^{N} \sum_{j=1, j \neq i}^{N} \alpha_{i j}\left(e_{i, t}-e_{j, t}\right)^{T} e_{i, t} \tilde{\gamma} d t & \\
+ & \frac{1}{2 N} \sum_{i=1}^{N} \sum_{j=1, j \neq i}^{N} \alpha_{i j} g_{r}\left(\left\|y_{i, t}-y_{j, t}\right\|\right)\left\|y_{i, t}-y_{j, t}\right\|^{2} \tilde{\gamma} d t \\
& \quad-\frac{1}{N} \sum_{i=1}^{N} \nabla^{T} f\left(y_{i, t}\right) e_{i, t} \tilde{\gamma} d t+\frac{\tau}{N} \tilde{\gamma} \sum_{i=1}^{N} d B_{i, t}^{T} e_{i, t}+\frac{m \tau^{2} \tilde{\gamma}^{2}(N-1)}{2 N} d t .
\end{aligned}
$$

Noticing that $g_{r}\left(\left\|y_{i, t}-y_{j, t}\right\|\right)\left\|y_{i, t}-y_{j, t}\right\|^{2} \leq b$, by (4.9) and (4.10),

$$
\begin{aligned}
d \bar{V}_{t} \leq & -2 a \lambda_{2} \tilde{\gamma} \bar{V}_{t} d t+\frac{b|\operatorname{Tr}(L)|}{2 N} \tilde{\gamma} d t+\frac{m \tau^{2} \tilde{\gamma}^{2}(N-1)}{2 N} d t+\frac{1}{N} \sum_{i=1}^{N} \eta\left\|e_{i, t}\right\| \tilde{\gamma} d t \\
& +\frac{\tau}{N} \tilde{\gamma} \sum_{i=1}^{N} d B_{i, t}{ }^{T} e_{i, t} \\
\leq & -2 a \lambda_{2} \tilde{\gamma} \bar{V}_{t} d t+\left[\frac{b|\operatorname{Tr}(L)|}{2 N} \tilde{\gamma}+\frac{m \tau^{2} \tilde{\gamma}^{2}(N-1)}{2 N}\right] d t+\sqrt{2} \eta \sqrt{\bar{V}} \tilde{\gamma} d t+\frac{\tau}{N} \tilde{\gamma} \sum_{i=1}^{N} d B_{i, t}{ }^{T} e_{i, t} \\
\leq & -c_{1} \tilde{\gamma} \bar{V}_{t} d t-\left(2 a \lambda_{2}-c_{1}\right) \tilde{\gamma} \bar{V}_{t} d t+\sqrt{2} \eta \sqrt{\bar{V}_{t}} \tilde{\gamma} d t+\left[\frac{b|T r(L)|}{2 N} \tilde{\gamma}+\frac{m \tau^{2} \tilde{\gamma}^{2}(N-1)}{2 N}\right] d t \\
& +\frac{\tau}{N} \tilde{\gamma} \sum_{i=1}^{N} d B_{i, t}{ }^{T} e_{i, t} \\
= & -c_{1} \tilde{\gamma} \bar{V}_{t} d t-\left(2 a \lambda_{2}-c_{1}\right)\left[\sqrt{\bar{V}}-\frac{\sqrt{2} \eta}{2\left(2 a \lambda_{2}-c_{1}\right)}\right]^{2} \tilde{\gamma} d t \\
& +\left[\frac{\eta^{2}}{2\left(2 a \lambda_{2}-c_{1}\right)} \tilde{\gamma}+\frac{b|T r(L)|}{2 N} \tilde{\gamma}+\frac{m \tau^{2} \tilde{\gamma}^{2}(N-1)}{2 N}\right] d t+\frac{\tau}{N} \tilde{\gamma} \sum_{i=1}^{N} d B_{i, t}^{T} e_{i, t} \\
\leq & -c_{1} \tilde{\gamma} \bar{V}_{t} d t+c_{2} \tilde{\gamma} d t+\frac{\tau}{N} \tilde{\gamma} \sum_{i=1}^{N} d B_{i, t}^{T} e_{i, t},
\end{aligned}
$$

where $c_{1} \in\left(0,2 a \lambda_{2}\right)$ is arbitrary, and

$$
c_{2}=\frac{\eta^{2}}{2\left(2 a \lambda_{2}-c_{1}\right)}+\frac{b|\operatorname{Tr}(L)|}{2 N}+\frac{m \tau^{2}(N-1)}{2 N} \tilde{\gamma}
$$


Applying Ito's formula to $e^{c_{1} \tilde{\gamma} t} \bar{V}_{t}$,

$$
d\left(e^{c_{1} \tilde{\gamma} t} \bar{V}_{t}\right)=e^{c_{1} \tilde{\gamma} t} d \bar{V}_{t}+c_{1} \tilde{\gamma} e^{c_{1} \tilde{\gamma} t} \bar{V}_{t} d t \leq c_{2} \tilde{\gamma} e^{c_{1} \tilde{\gamma} t} d t+\frac{\tau}{N} \tilde{\gamma} e^{c_{1} \tilde{\gamma} t} \sum_{i=1}^{N} d B_{i, t}{ }^{T} e_{i, t} .
$$

Integrating the stochastic differential inequality,

$$
\bar{V}_{t} \leq e^{-c_{1} \tilde{\gamma} t} \bar{V}_{0}+\frac{c_{2}}{c_{1}}\left(1-e^{-c_{1} \tilde{\gamma} t}\right)+e^{-c_{1} \tilde{\gamma} t} \int_{0}^{t} \frac{\tau}{N} \tilde{\gamma} e^{c_{1} \tilde{\gamma} s} \sum_{i=1}^{N} d B_{i, s}{ }^{T} e_{i, s}
$$

Taking an ensemble average on both sides yields

$$
\mathbb{E}\left[\bar{V}_{t}\right] \leq e^{-c_{1} \tilde{\gamma} t} \bar{V}_{0}+\frac{c_{2}}{c_{1}}\left(1-e^{-c_{1} \tilde{\gamma} t}\right)
$$

It follows that

$$
\mathbb{E}\left[\bar{V}_{t}\right] \leq\left(\bar{V}_{0}-\frac{c_{2}}{c_{1}}\right) e^{-c_{1} \tilde{\gamma} t}+\frac{c_{2}}{c_{1}} \leq \begin{cases}\bar{V}_{0} & \text { if } \bar{V}_{0} \geq \frac{c_{2}}{c_{1}} \\ \frac{c_{2}}{c_{1}} & \text { if } \bar{V}_{0}<\frac{c_{2}}{c_{1}}\end{cases}
$$

Therefore

$$
\mathbb{E}\left[\bar{V}_{t}\right] \leq \max \left\{\bar{V}_{0}, \frac{c_{2}}{c_{1}}\right\}, \forall t
$$

In the long run,

$$
\mathbb{E}\left[\bar{V}_{t}\right] \leq \frac{c_{2}}{c_{1}}=\frac{\eta^{2}}{2 c_{1}\left(2 a \lambda_{2}-c_{1}\right)}+\frac{b|\operatorname{Tr}(L)|}{2 c_{1} N}+\frac{m \tau^{2}(N-1)}{2 c_{1} N} \tilde{\gamma} .
$$

Notice that the above inequality is valid for all $c_{1} \in\left(0,2 a \lambda_{2}\right)$, of which we look for the minimum over all possible $c_{1}$ 's. Define

$$
\begin{aligned}
\psi\left(c_{1}\right) & =\frac{\eta^{2}}{2 c_{1}\left(2 a \lambda_{2}-c_{1}\right)}+\frac{b|\operatorname{Tr}(L)|}{2 c_{1} N}+\frac{m \tau^{2}(N-1)}{2 c_{1} N} \tilde{\gamma} \\
& =\left[\frac{\eta^{2}}{4 a \lambda_{2}}+\frac{b|\operatorname{Tr}(L)|}{2 N}+\frac{m \tau^{2}(N-1)}{2 N} \tilde{\gamma}\right] \frac{1}{c_{1}}+\frac{\eta^{2}}{4 a \lambda_{2}} \frac{1}{\left(2 a \lambda_{2}-c_{1}\right)}
\end{aligned}
$$

By Cauchy-Schwartz inequality, when

$$
\frac{1}{c_{1}}=\frac{1}{2 a \lambda_{2}}\left(1+\sqrt{\frac{c_{4}}{c_{3}}}\right)
$$


$\psi\left(c_{1}\right)$ attains its minimum

$$
\psi^{*}=\frac{1}{2 a \lambda_{2}}\left(\sqrt{c_{3}}+\sqrt{c_{4}}\right)^{2} .
$$

Here

$$
c_{3}=\frac{\eta^{2}}{4 a \lambda_{2}}+\frac{b|\operatorname{Tr}(L)|}{2 N}+\frac{m \tau^{2}(N-1)}{2 N} \tilde{\gamma}, c_{4}=\frac{\eta^{2}}{4 a \lambda_{2}}
$$

Since (4.33) is valid for all $c_{1} \in\left(0,2 a \lambda_{2}\right)$, it holds true that $\mathbb{E}\left[\bar{V}_{t}\right] \leq \psi^{*}$ in the long run.

\subsubsection{Proof of Theorem 4}

\section{Preliminaries}

According to Equation (4.26) and Ito's lemma,

$$
\begin{aligned}
d U_{t}= & d\left(\bar{x}_{t}-x^{*}\right) \cdot\left(\bar{x}_{t}-x^{*}\right)+\frac{1}{2} d\left(\bar{x}_{t}-x^{*}\right) \cdot d\left(\bar{x}_{t}-x^{*}\right) \\
= & -\frac{1}{N} \sum_{i=1}^{N} \nabla^{T} f\left(y_{i, t}\right)\left(\bar{x}_{t}-x^{*}\right) \tilde{\gamma} d t+\frac{\tau}{N} \tilde{\gamma} \sum_{i=1}^{N} d B_{i, t}^{T}\left(\bar{x}_{t}-x^{*}\right)+\frac{m \tau^{2} \tilde{\gamma}^{2}}{2 N} d t \\
= & -\frac{1}{N} \sum_{i=1}^{N} \nabla^{T} f\left(y_{i, t}\right)\left(y_{i, t}-x^{*}\right) \tilde{\gamma} d t+\frac{1}{N} \sum_{i=1}^{N} \nabla^{T} f\left(y_{i, t}\right) e_{i, t} \tilde{\gamma} d t+\frac{\tau}{N} \tilde{\gamma} \sum_{i=1}^{N} d B_{i, t}^{T}\left(\bar{x}_{t}-x^{*}\right) \\
& +\frac{m \tau^{2} \tilde{\gamma}^{2}}{2 N} d t .
\end{aligned}
$$

Since $f(\cdot)$ attains its minimum at $x^{*}, \nabla f\left(x^{*}\right)=0$. Then by Assumption 4 and Equation (4.13),

$$
\begin{aligned}
-\frac{1}{N} \sum_{i=1}^{N} \nabla^{T} f\left(y_{i, t}\right)\left(y_{i, t}-x^{*}\right) & =-\frac{1}{N} \sum_{i=1}^{N}\left(\nabla^{T} f\left(y_{i, t}\right)-\nabla^{T} f\left(x^{*}\right)\right)\left(y_{i, t}-x^{*}\right) \\
& \leq-2 \kappa \bar{F}_{t}=-2 \kappa\left(U_{t}+\bar{V}_{t}\right) .
\end{aligned}
$$

By Assumption 5,

$$
\frac{1}{N} \sum_{i=1}^{N} \nabla^{T} f\left(y_{i, t}\right) e_{i, t}=\frac{1}{N} \sum_{i=1}^{N}\left(\nabla^{T} f\left(y_{i, t}\right)-\nabla^{T} f\left(\bar{x}_{t}\right)\right)\left(y_{i, t}-\bar{x}_{t}\right) \leq \frac{1}{N} \sum_{i=1}^{N} \mu\left\|e_{i, t}\right\|^{2}=2 \mu \bar{V}_{t} .
$$


Therefore,

$$
\begin{aligned}
d U_{t} & \leq-2 \kappa \tilde{\gamma}\left(U_{t}+\bar{V}_{t}\right) d t+2 \mu \tilde{\gamma} \bar{V}_{t} d t+\frac{m \tau^{2} \tilde{\gamma}^{2}}{2 N} d t+\frac{\tau}{N} \tilde{\gamma} \sum_{i=1}^{N} d B_{i, t}{ }^{T} \bar{x}_{t} \\
& =-2 \kappa \tilde{\gamma} U_{t} d t+2(\mu-\kappa) \tilde{\gamma} \bar{V}_{t} d t+\frac{m \tau^{2} \tilde{\gamma}^{2}}{2 N} d t+\frac{\tau}{N} \tilde{\gamma} \sum_{i=1}^{N} d B_{i, t}{ }^{T} \bar{x}_{t}
\end{aligned}
$$

Lemma 2.

$$
d \bar{V}_{t} \leq-2 a \lambda_{2} \tilde{\gamma} \bar{V}_{t} d t-2 \kappa \tilde{\gamma} \bar{V}_{t} d t+\frac{m \tau^{2} \tilde{\gamma}^{2}(N-1)}{2 N} d t+\frac{\tau}{N} \tilde{\gamma} \sum_{i=1}^{N} d B_{i, t}{ }^{T} e_{i, t}
$$

Proof. By Equation (4.12),

$$
\begin{array}{r}
d \bar{V}_{t}=-\frac{a}{N} \sum_{i=1}^{N} \sum_{j=1, j \neq i}^{N} \alpha_{i j}\left(e_{i, t}-e_{j, t}\right)^{T} e_{i} \tilde{\gamma} d t-\frac{1}{N} \sum_{i=1}^{N} \nabla^{T} f\left(y_{i, t}\right) e_{i, t} \tilde{\gamma} d t+\frac{\tau}{N} \tilde{\gamma} \sum_{i=1}^{N} d B_{i, t}^{T} e_{i, t} \\
+\frac{m \tau^{2} \tilde{\gamma}^{2}(N-1)}{2 N} d t
\end{array}
$$

Notice that

$$
\sum_{i=1}^{N} \nabla^{T} f\left(y_{i, t}\right) e_{i, t}=\sum_{i=1}^{N}\left(\nabla^{T} f\left(y_{i, t}\right)-\nabla^{T} f\left(\bar{x}_{t}\right)\right)\left(y_{i, t}-\bar{x}_{t}\right) \geq \sum_{i=1}^{N} \kappa\left\|y_{i, t}-\bar{x}_{t}\right\|^{2}=2 \kappa N \bar{V}_{t}
$$

where the inequality follows from Assumption 4. In light of (4.9) and (4.10),

$$
d \bar{V}_{t} \leq-2 a \lambda_{2} \bar{V}_{t} \tilde{\gamma} d t-2 \kappa \bar{V}_{t} \tilde{\gamma} d t++\frac{m \tau^{2} \tilde{\gamma}^{2}(N-1)}{2 N} d t+\frac{\tau}{N} \tilde{\gamma} \sum_{i=1}^{N} d B_{i, t}{ }^{T} e_{i, t}
$$

\section{Proof of Theorem 4}

Define

$$
W_{t}=U_{t}+\frac{(\mu-\kappa)}{a \lambda_{2}} \bar{V}_{t}
$$


By (4.35) and (4.36),

$$
\begin{aligned}
d W_{t} \leq & -2 \kappa \tilde{\gamma} U_{t} d t+\left[2(\mu-\kappa)-\frac{(\mu-\kappa)}{a \lambda_{2}}\left(2 \kappa+2 a \lambda_{2}\right)\right] \tilde{\gamma} \bar{V}_{t} d t+\frac{m \tau^{2} \tilde{\gamma}^{2}}{2 N} d t \\
& +\frac{m \tau^{2} \tilde{\gamma}^{2}(\mu-\kappa)(N-1)}{2 a N \lambda_{2}} d t+\frac{\tau}{N} \tilde{\gamma} \sum_{i=1}^{N} d B_{i, t}{ }^{T} \bar{x}_{t}+\frac{\tau(\mu-\kappa)}{a N \lambda_{2}} \tilde{\gamma} \sum_{i=1}^{N} d B_{i, t}{ }^{T} e_{i, t} \\
= & -2 \kappa \tilde{\gamma} W_{t} d t+\frac{m \tau^{2} \tilde{\gamma}^{2}}{2 N}\left[1+\frac{(\mu-\kappa)(N-1)}{a \lambda_{2}}\right] d t+\frac{\tau}{N} \tilde{\gamma} \sum_{i=1}^{N} d B_{i, t}{ }^{T} \bar{x}_{t} \\
& +\frac{\tau(\mu-\kappa)}{a N \lambda_{2}} \tilde{\gamma} \sum_{i=1}^{N} d B_{i, t}{ }^{T} e_{i, t} .
\end{aligned}
$$

Then,

$$
\begin{aligned}
& d\left(e^{2 \kappa \tilde{\gamma} t} W_{t}\right) \\
& =e^{2 \kappa \tilde{\gamma} t} d W_{t}+2 \kappa \tilde{\gamma} e^{2 \kappa \tilde{\gamma} t} W_{t} d t \\
& \leq \frac{m \tau^{2} \tilde{\gamma}^{2}}{2 N}\left[1+\frac{(\mu-\kappa)(N-1)}{a \lambda_{2}}\right] e^{2 \kappa \tilde{\gamma} t} d t+\frac{\tau}{N} \tilde{\gamma} e^{2 \kappa \tilde{\gamma} t}\left[\sum_{i=1}^{N} d B_{i, t}{ }^{T} \bar{x}_{t}+\frac{(\mu-\kappa)}{a \lambda_{2}} \sum_{i=1}^{N} d B_{i, t}{ }^{T} e_{i, t}\right] .
\end{aligned}
$$

Integrating both sides yields

$$
\begin{aligned}
W_{t} \leq e^{-2 \kappa \tilde{\gamma} t} W_{0}+\frac{m \tau^{2} \tilde{\gamma}}{4 \kappa N}[1 & \left.+\frac{(\mu-\kappa)(N-1)}{a \lambda_{2}}\right]\left(1-e^{-2 \kappa \tilde{\gamma} t}\right) \\
& +e^{-2 \kappa \tilde{\gamma} t} \int_{0}^{t} \frac{\tau}{N} \tilde{\gamma} e^{2 \kappa \tilde{\gamma} s}\left[\sum_{i=1}^{N} d B_{i, s}{ }^{T} \bar{x}_{s}+\frac{(\mu-\kappa)}{a \lambda_{2}} \sum_{i=1}^{N} d B_{i, s}{ }^{T} e_{i, s}\right] .
\end{aligned}
$$

Taking ensemble average, we get

$$
\mathbb{E}\left[W_{t}\right] \leq e^{-2 \kappa \tilde{\gamma} t} W_{0}+\frac{m \tau^{2} \tilde{\gamma}}{4 \kappa N}\left[1+\frac{(\mu-\kappa)(N-1)}{a \lambda_{2}}\right]\left(1-e^{-2 \kappa \tilde{\gamma} t}\right) .
$$

By (4.39),

$$
\mathbb{E}\left[U_{t}\right] \leq \mathbb{E}\left[W_{t}\right] \leq e^{-2 \kappa \tilde{\gamma} t}\left[U_{0}+\frac{(\mu-\kappa)}{a \lambda_{2}} \bar{V}_{0}\right]+\frac{m \tau^{2} \tilde{\gamma}}{4 \kappa N}\left[1+\frac{(\mu-\kappa)(N-1)}{a \lambda_{2}}\right]\left(1-e^{-2 \kappa \tilde{\gamma} t}\right) .
$$

In the long run,

$$
\mathbb{E}\left[U_{t}\right] \leq \frac{m \tau^{2} \tilde{\gamma}}{4 \kappa N}\left[1+\frac{(\mu-\kappa)(N-1)}{a \lambda_{2}}\right]
$$




\subsubsection{Proof of Proposition 1}

By (4.16) and (4.19),

$$
\frac{1}{2} \mathbb{E}\left[\left\|x_{t}-x^{*}\right\|^{2}\right] \geq e^{-2 \mu \gamma \Gamma t} G_{0}+\frac{m \tau_{N}^{2} \gamma}{4 \mu}\left(1-e^{-2 \mu \gamma \Gamma t}\right)
$$

and

$$
\frac{1}{2} \mathbb{E}\left[\left\|\bar{x}_{t}-x^{*}\right\|^{2}\right] \leq e^{-2 \kappa \tilde{\gamma} \tilde{\Gamma} t}\left[U_{0}+\frac{(\mu-\kappa)}{a \lambda_{2}} \bar{V}_{0}\right]+\frac{m \tau^{2} \tilde{\gamma}}{4 \kappa N}\left[1+\frac{(\mu-\kappa)(N-1)}{a \lambda_{2}}\right]\left(1-e^{-2 \kappa \tilde{\gamma} \tilde{\Gamma} t}\right) .
$$

Let

$$
d_{1}=\frac{m \tau_{N}^{2} \gamma}{4 \mu}, \quad d_{2}=U_{0}+\frac{(\mu-\kappa)}{a \lambda_{2}} \bar{V}_{0}
$$

and

$$
d_{3}=\frac{m \tau^{2} \tilde{\gamma}}{4 \kappa N}\left[1+\frac{(\mu-\kappa)(N-1)}{a \lambda_{2}}\right]
$$

It follows that

$$
\mathbb{E}\left[\left\|x_{t}-x^{*}\right\|^{2}\right] \geq 2\left(G_{0}-d_{1}\right) e^{-2 \mu \gamma \Gamma t}+2 d_{1}
$$

and

$$
\mathbb{E}\left[\left\|\bar{x}_{t}-x^{*}\right\|^{2}\right] \leq 2\left(d_{2}-d_{3}\right) e^{-2 \kappa \tilde{\gamma} \tilde{\Gamma} t}+2 d_{3} .
$$

From the discussion in Section 4.4.1, $d_{1} \sim 1 / N^{(1 / \beta)-1}$ and $d_{3} \sim 1 / N$. Therefore, there exists $N^{*}$ such that when $N>N^{*}, d_{3}<d_{1}$. In this case, Let $t^{*}$ be such that

$$
d_{1}-d_{3}=\left|G_{0}-d_{1}\right| e^{-2 \mu \gamma \Gamma t^{*}}+\left|d_{2}-d_{3}\right| e^{-2 \kappa \tilde{\gamma} \tilde{\Gamma} t^{*}}
$$

Then for all $t>t^{*}$,

$$
\mathbb{E}\left[\left\|\bar{x}_{t}-x^{*}\right\|^{2}\right]<\mathbb{E}\left[\left\|x_{t}-x^{*}\right\|^{2}\right]
$$

\subsubsection{Proof of Theorem 5}

We start by looking for the joint density of $\left(\bar{y}^{T}, e_{1}^{T}, \ldots, e_{N-1}^{T}\right)$. Notice that $\left(\bar{y}^{T}, e_{1}^{T}, \ldots, e_{N-1}^{T}\right)=$ $\left(D \otimes I_{m}\right) \mathbf{y}_{t}$, where $D$ is a $N \times N$ matrix. It is easy to verify that $D$ has full rank, so that 
$\left(D \otimes I_{m}\right)^{-1}$ exists. It follows that (see Jacod and Protter [45])

$$
\begin{aligned}
\pi\left(\bar{y}^{T}, e_{1}^{T}, \ldots, e_{N-1}^{T}\right) & =\frac{1}{\operatorname{det}\left(D \otimes I_{m}\right)} \pi\left(\left(D \otimes I_{m}\right)^{-1}\left(\bar{y}^{T}, e_{1}^{T}, \ldots, e_{N-1}^{T}\right)\right) \\
& =\frac{1}{\tilde{K}} \exp \left\{-2\left[\sum_{i=1}^{N} f\left(\bar{y}+e_{i}\right)+\frac{1}{2} \sum_{i=1}^{N} \sum_{j=1, j \neq i}^{N} \alpha_{i j} J\left(\left\|e_{i, t}-e_{j, t}\right\|\right)\right] /\left(\tau^{2} \tilde{\gamma}\right)\right\} .
\end{aligned}
$$

where $\tilde{K}$ is a normalizing factor. The density of $\bar{y}$ is calculated as

$$
\pi(\bar{y})=\frac{1}{\tilde{K}} \int \exp \left\{-2\left[\sum_{i=1}^{N} f\left(\bar{y}+e_{i}\right)+\frac{1}{2} \sum_{i=1}^{N} \sum_{j=1, j \neq i}^{N} \alpha_{i j} J\left(\left\|e_{i, t}-e_{j, t}\right\|\right)\right] /\left(\tau^{2} \tilde{\gamma}\right)\right\} d e_{1} \cdots d e_{N-1} .
$$

Notice that $e_{N}=-\sum_{i=1}^{N-1} e_{i}$ in the equation above. We rewrite the potential function $J$ as a sum of two parts: $J=J_{a}+J_{r}$, where $\nabla_{x} J_{a}(\|x\|)=x g_{a}(\|x\|)=a x$, and $\nabla_{x} J_{r}(\|x\|)=$ $x g_{r}(\|x\|)$. Without loss of generality, we assume that $J_{a}(\|x\|)=(1 / 2) a\|x\|^{2}$. Then (refer to Godsil and Royle [36])

$$
\begin{aligned}
& \pi(\bar{y}) \\
& =\frac{1}{\tilde{K}} \int \exp \left\{-2\left[\sum_{i=1}^{N} f\left(\bar{y}+e_{i}\right)+\frac{1}{2} \sum_{i=1}^{N} \sum_{j=1, j \neq i}^{N} \alpha_{i j} \frac{a}{2}\left\|e_{i}-e_{j}\right\|^{2}+R\right] /\left(\tau^{2} \tilde{\gamma}\right)\right\} d e_{1} \cdots d e_{N-1} \\
& =\frac{1}{\tilde{K}} \int \exp \left\{-2\left[\sum_{i=1}^{N} f\left(\bar{y}+e_{i}\right)+\frac{a}{2} \mathbf{e}^{T}\left(L \otimes I_{m}\right) \mathbf{e}+R\right] /\left(\tau^{2} \tilde{\gamma}\right)\right\} d e_{1} \cdots d e_{N-1},
\end{aligned}
$$

where

$$
R=\frac{1}{2} \sum_{i=1}^{N} \sum_{j=1, j \neq i}^{N} \alpha_{i j} J_{r}\left(\left\|e_{i, t}-e_{j, t}\right\|\right)
$$

Let $z_{i}=a^{1 / 2} e_{i}, \forall i$.

$$
\begin{aligned}
& \int \exp \left\{-2\left[\sum_{i=1}^{N} f\left(\bar{y}+e_{i}\right)+\frac{a}{2} \mathbf{e}^{T}\left(L \otimes I_{m}\right) \mathbf{e}+R\right] /\left(\tau^{2} \tilde{\gamma}\right)\right\} d e_{1} \cdots d e_{N-1} \\
& =a^{-(N-1) / 2} \int \exp \left\{-2\left[\sum_{i=1}^{N} f\left(\bar{y}+a^{-1 / 2} z_{i}\right)+\frac{1}{2} \mathbf{z}^{T}\left(L \otimes I_{m}\right) \mathbf{z}+\tilde{R}\right] /\left(\tau^{2} \tilde{\gamma}\right)\right\} d z_{1} \cdots d z_{N-1} .
\end{aligned}
$$

Here

$$
\tilde{R}=\frac{1}{2} \sum_{i=1}^{N} \sum_{j=1, j \neq i}^{N} \alpha_{i j} J_{r}\left(a^{-1 / 2}\left\|z_{i, t}-z_{j, t}\right\|\right)
$$


Given that graph $\mathcal{G}$ is connected, $\mathbf{z}^{T}\left(L \otimes I_{m}\right) \mathbf{z} \geq \lambda_{2} \mathbf{e}^{T} \mathbf{e}$ with $\lambda_{2}>0$. Then since $f$ and $J_{r}$ are continuous, we have

$$
\begin{aligned}
& \lim _{a \rightarrow \infty} \int \exp \left\{-2\left[\sum_{i=1}^{N} f\left(\bar{y}+a^{-1 / 2} z_{i}\right)+\frac{1}{2} \mathbf{z}^{T}\left(L \otimes I_{m}\right) \mathbf{z}+\tilde{R}\right] /\left(\tau^{2} \tilde{\gamma}\right)\right\} d z_{1} \cdots d z_{N-1} \\
& =\int \exp \left\{-2\left[N f(\bar{y})+\frac{1}{2} \mathbf{z}^{T}\left(L \otimes I_{m}\right) \mathbf{z}+\tilde{R}_{0}\right] /\left(\tau^{2} \tilde{\gamma}\right)\right\} d z_{1} \cdots d z_{N-1} \\
& =\exp \left\{-2 N f(\bar{y}) /\left(\tau^{2} \tilde{\gamma}\right)\right\} \exp \left\{-2 \tilde{R}_{0} /\left(\tau^{2} \tilde{\gamma}\right)\right\} \int \exp \left\{\mathbf{z}^{T}\left(L \otimes I_{m}\right) \mathbf{z} /\left(\tau^{2} \tilde{\gamma}\right)\right\} d z_{1} \cdots d z_{N-1},
\end{aligned}
$$

where

$$
\tilde{R}_{0}=\frac{1}{2} \sum_{i=1}^{N} \sum_{j=1, j \neq i}^{N} \alpha_{i j} J_{r}(0)=\frac{1}{2}|\operatorname{Tr}(L)| J_{r}(0) .
$$

Therefore,

$$
\begin{aligned}
& \lim _{a \rightarrow \infty} \pi(\bar{y}) \\
& =\lim _{a \rightarrow \infty} \frac{1}{\tilde{K}} \int \exp \left\{-2\left[\sum_{i=1}^{N} f\left(\bar{y}+e_{i}\right)+\frac{1}{2} \sum_{i=1}^{N} \sum_{j=1, j \neq i}^{N} \alpha_{i j} J\left(\left\|e_{i, t}-e_{j, t}\right\|\right)\right] /\left(\tau^{2} \tilde{\gamma}\right)\right\} d e_{1} \cdots d e_{N-1} \\
& =\frac{\exp \left\{-2 N f(\bar{y}) /\left(\tau^{2} \tilde{\gamma}\right)\right\} \exp \left\{-2 \tilde{R}_{0} / \tau^{2}\right\} \int \exp \left\{\mathbf{z}^{T}\left(L \otimes I_{m}\right) \mathbf{z} /\left(\tau^{2} \tilde{\gamma}\right)\right\} d z_{1} \cdots d z_{N-1}}{\int \exp \left\{-2 N f(\bar{y}) /\left(\tau^{2} \tilde{\gamma}\right)\right\} \exp \left\{-2 \tilde{R}_{0} /\left(\tau^{2} \tilde{\gamma}\right)\right\} \int \exp \left\{\mathbf{z}^{T}\left(L \otimes I_{m}\right) \mathbf{z} /\left(\tau^{2} \tilde{\gamma}\right)\right\} d z_{1} \cdots d z_{N-1} d \bar{y}} \\
& =\frac{\exp \left\{-2 N f(\bar{y}) /\left(\tau^{2} \tilde{\gamma}\right)\right\}}{\int \exp \left\{-2 N f(\bar{y}) /\left(\tau^{2} \tilde{\gamma}\right)\right\} d \bar{y}} \\
& =\frac{\exp \left\{-2 N f(\bar{y}) /\left(\sigma^{2} \tilde{\Gamma}\right)\right\}}{\int \exp \left\{-2 N f(\bar{y}) /\left(\sigma^{2} \tilde{\Gamma}\right)\right\} d \bar{y}} .
\end{aligned}
$$

This completes the proof. 


\section{CHAPTER 5}

\section{Iterative Mechanisms for Electricity Markets}

In this chapter, we consider the problem of designing the rules by which market dispatch and payment to participants are gradually adjusted while taking into account network and reliability constraints so as to ensure the market clears with an efficient outcome. Small adjustments (which require minimal information from market participants at each iteration) facilitate the identification of incentives for ensuring truthful reporting of private information. We propose a class of iterative mechanisms and show this class exhibits many desirable properties (approximately): incentive compatibility, efficiency, individual rationality and (weak) budget balance. In the second part of this chapter, we analyze an iterative mechanism for stochastic market clearing, a pressing need given the increasing penetration of highly intermittent renewable generation technologies. In this case, the marginal cost of adjustments may only be estimated with some error. We show that truthful reporting is a Nash equilibrium and the resulting dispatch converges almost surely to the efficient dispatch.

\section{$5.1 \quad$ Introduction}

Electricity is a complex bundle of services involving multiple power plants and consumers in various locations over a capacity constrained network. A particular feature of many electricity markets is that market clearing is carried out by solving an optimization problem using input information (e.g., bids to buy and/or sell electricity) from market participants. This type of clearing is needed because other more traditional clearing protocols (e.g., bilateral 
trading) may not reliably or timely satisfy physical constraints. Market clearing is thus akin to a "black box" since the underlying optimization model is not necessarily transparent to market participants. The use of relatively sophisticated "black box" optimization models for clearing has important implications for market design. Some market participants because of their relative size, location or cost may have the ability to significantly alter market clearing outcomes by manipulating the information they provide to the ISO/RTO in charge of clearing. Depending upon the pricing rules in place, some market participants may find it advantageous to manipulate market clearing outcomes. This could possibly result in inefficient outcomes as well as distorted prices which provide incorrect investment incentives. Because of the "black box" nature of market clearing, it may be exceedingly hard to detect and/or identify certain types of market power abuse.

A cursory look at the history of commodity markets highlights the ways in which the history of electricity markets is fundamentally different. Most commodity markets gradually evolved towards standard terms of trade and institutions that helped increase liquidity and reduce transaction risks (see for example McMillan [63]). In contrast, most electricity markets artificially evolved from the coordinated or pooled operation of vertically integrated (and highly regulated) utilities. This evolution was rather artificial in that it was imposed by governments intent on bringing an element of competition to an industry no longer considered a natural monopoly. Not surprisingly many alternative designs for the operation of electricity markets have been tried out by different countries. At times, concerns over economic efficiency have motivated comprehensive reforms. This was the case of the NETA reform in the UK (see $[26,48]$ ) according to which a uniform pricing rule was replaced by pay-as-bid pricing rule (among other important changes). More than thirty years after the first electricity markets began operating, the task of properly tuning market rules to ensure economically efficient outcomes (i.e., market design) seems far from complete. ${ }^{1}$

The identification of the optimal design for an electricity market can be formulated as a mechanism design problem (see Silva et al. [87]). Mechanism design is a field of game theory

\footnotetext{
${ }^{1}$ See for example the discussion prompted by FERC's Notice of Proposed Rulemaking Docket No. RM165-000 regarding the relaxation of the price cap.
} 
in which the rules of a game are designed in order to induce a desired equilibrium outcome resulting from the interaction of rational agents with private information (see Borgers et al. [8]). In a direct mechanism, market participants (i.e., generators and retailers) report their private information (i.e., costs and willingness to pay) to the ISO or RTO which in turn clears the market and determines the associated market compensation and/or charges for each market participant. A mechanism is said to be incentive compatible in dominant strategies (or strategy-proof) if it is optimal for every market participant to truthfully report their private information regardless of the reporting strategies of other participants. A mechanism is said to be efficient if the market clears with the efficient market outcome. A weaker form of incentive compatibility is related to implementation in a Bayes-Nash equilibrium. This concept relies on a common knowledge assumption on the market participants' private information (see Borgers et al. [8]).

While direct mechanisms only require one round of interaction, the nature of the information exchange between market participants and the market-maker is quite complex. For example, in many designs currently in place, each generator (respectively, each retailer) must report their complete cost function (respectively, their willingness to pay) over the set of individual market outcomes. In this rich strategy space, equilibrium strategic behavior can be quite complex often inducing inefficient market outcomes (see for example, Anderson et al. [2] and Holmberg and Newbery [41]). In this chapter, we consider the problem of designing a mechanism, i.e., the rules by which market dispatch and payment to participants in an electricity are determined while taking into account network and reliability constraints. Our approach differs from Silva et al. [87] and the more recent work of Xu and Low [110] and Jofré et al. [47] in that we consider iterative mechanisms. In an iterative mechanism, market participants are asked to report the cost or willingness to pay for small adjustments at each iteration. A tentative market dispatch is marginally adjusted ensuring network constraints are satisfied and a stopping criteria is checked. At each iteration, each market participant is charged (or compensated) according to the marginal change in surplus to all other market participants. This pricing rule can be seen as a marginal implementation of Clarke-Groves 
mechanism (see $[17,37])$. Small adjustments requiring minimal information from market participants at each iteration, facilitate the identification of incentives for ensuring truthful reporting of private information. To see why this is the case, consider the case of a demand aggregator. Reporting a lower than truthful marginal valuation leads to a marginal reduction in the tentatively dispatched demand. The compensation for this reduction is equal to the cost savings associated to marginally decreased generation net of any increased marginal surplus for other demand aggregators. Similarly, reporting a higher than truthful marginal valuation leads to a marginal increase in the tentatively dispatched demand. The payment for this increase is equal to the cost increase associated to marginally increased generation net of any reduced marginal surplus for other demand aggregators. Thus, for small adjustments, the incentives of the individual demand aggregator are aligned with those of the marketmaker which aims to locally improve the efficiency of the tentative dispatch. However, this marginal incentive compatibility property may not be robust to the possibilities of dynamic strategic behavior that an iterative mechanism offers. We address this concern by incorporating two design features. First, we assume the information regarding the tentative schedule adjustments are privately communicated by the market-maker to each market participant. This information flow precludes the possibility of statistical learning by any given participant regarding the likely types of other market participants. Secondly, in the class of iterative mechanisms proposed, the market-maker checks (at every iteration) an activity rule for each market participant. This ensures that the reporting strategies by a generator (respectively, demand aggregator) must be consistent over time with some convex cost function (respectively, concave valuation function). These design features allow the extension of the local incentive compatibility property to a more complex (infinite dimensional) strategy space. We show that truthful reporting is (approximately) a dominant strategy. Upon stopping, the resulting economic dispatch is approximately efficient. Individual participation constraints are satisfied as the net surplus from trade is approximately shared equally by all participants and the mechanism is approximately weakly budget balanced. Interestingly, the mechanism may potentially be suitably altered in various ways as its main properties are not dependent 
upon the closed-form representation of network constraints but rather on the convexity of the set of feasible solutions. For example, the proposed mechanism may potentially be used in conjunction with convex approximations to AC power flow (see [84], [24]).

In the second part of the chapter we consider an iterative mechanism for stochastic market clearing. The inherent intermittency of renewable technologies poses new challenges for ensuring a reliable and secure operation of electricity markets. As the marginal cost of renewable electricity is negligible, purely economic considerations would dictate a market clearing outcome in which most renewable output is cleared. However, intermittency may compromise the secure operation of the power grid infrastructure supporting the market. The incorporation of reliability and/or security constraints in market clearing further complicates the task of market design. In the second part of the chapter, we consider an iterative mechanism for stochastic market clearing (see $[10,78,107,113])$. This is an important feature as the share of renewable (intermittent) capacity in most electricity markets around the world is increasing at a steady pace and ex-post adjustments to market dispatch are the norm. In a direct mechanism for stochastic market clearing, the market-maker selects both a nominal dispatch and (ex-post) dispatch adjustments based upon the reports by market participants regarding their cost and valuation structure (including the cost of adjustments). In this context, equilibrium strategic behavior can be quite complex so that a comparison between different direct mechanism designs is not straightforward (see Khazaei et al. [49]). We propose an iterative mechanism for stochastic market clearing. In this context, participants may report incorrect values for the marginal expected cost while still being truthful. This is due to estimation error. We show that truthful reporting is a Nash equilibrium and the resulting dispatch converges almost surely to the efficient dispatch.

The structure of this chapter is as follows. In Section 2 we introduce the basic economic dispatch problem with network constraints. In Section 3, we introduce an iterative mechanism design and discuss its main features. In Section 4 we analyze the mechanism's main properties: convergence, efficiency, incentive compatibility and budget balance. We also discuss other iterative mechanisms that could exhibit similar properties. In Section 5 we 
introduce an iterative mechanism for stochastic market clearing and analyze its properties. Finally we close in Section 6 with conclusions and a discussion of further work.

\subsection{Preliminaries}

\subsubsection{Constrained Economic Dispatch}

We consider a stylized description of the day-ahead market clearing for a given hour in an electricity market. For ease of exposition, we will assume in what follows that there is a single demand aggregator (e.g. retailer) at each node $i \in \mathcal{I}$ in the network. We shall denote by $d_{i}$ (in $M W / h r$ ) demand aggregator $i$ 's demand and $v_{i}(d)$ is demand aggregator $i$ 's valuation for

consumption level $d \geq 0$. We also assume there is at most one generator per node with total $\operatorname{cost} C_{i}(x)$ for a production level $x \geq 0$. The assumption of a single retailer and generator at each node will be relaxed later (see Appendix B) so that both retailers and generators may simultaneously operate in several nodes.

The shift-factor formulation of optimal DC power flow is given by:

$$
\left\{\begin{aligned}
\max \sum_{i}\left[v_{i}\left(d_{i}\right)-C_{i}\left(x_{i}\right)\right] & \\
\text { s.t } & \sum_{i}\left(x_{i}-d_{i}\right) \\
-z_{\ell} \leq \sum_{i} \eta_{\ell, i}\left(x_{i}-d_{i}\right) & \leq z_{\ell}(b) \\
\underline{x}_{i} \leq x_{i} & \leq \bar{x}_{i}(c) \\
d_{i} & \geq 0 \quad(d)
\end{aligned}\right.
$$

where $\eta_{\ell, i} \in[-1,1]$ is the power shift factor from node $i$ to line $\ell, z_{\ell}$ is the capacity of line $\ell$ and $\underline{x}_{i}, \bar{x}_{i}$ are the lower and upper limits for generator $i$.

\subsubsection{Mechanism Design}

Information on the functions $v_{i}$ and $C_{i}$ is private to demand aggregators and generators. In a typical market design, market participants report functions $\hat{v}_{i}$ and $\hat{C}_{i}$ to the market- 
maker (ISO or RTO) so that locational marginal prices are identified by solving problem (5.1). However, there is no guarantee that $\hat{v}_{i}=v_{i}$ and $\hat{C}_{i}=C_{i}$, i.e. market participants may not truthfully reveal their private information. This implies the resulting dispatch is not necessarily efficient. In what follows we will present and analyze a mechanism that guarantees market participants (demand aggregators and generators) report truthful information to the market maker in order to identify the optimal solution to problem (5.1).

To model private information, let $\mathcal{V}_{i}$ denote the type space for demand aggregator located in node $i \in \mathcal{I}$. Similarly, let $\mathcal{C}_{i}$ denote the type space for generator located in node $i \in \mathcal{I}$. We make the following standing assumption:

Assumption 6. For each node i, $\mathcal{C}_{i}$ (respectively, $\mathcal{V}_{i}$ ) is the set of strictly convex (respectively, strictly concave) Lipschitz functions with continuous derivatives.

Denote the total number of nodes by $I=|\mathcal{I}|$. Let $\mathbf{x}=\left[x_{1}, x_{2}, \ldots, x_{I}\right]$ and $\mathbf{d}=$ $\left[d_{1}, d_{2}, \ldots, d_{I}\right]$. In a direct mechanism, each generator reports a type $\hat{C}_{i} \in \mathcal{C}_{i}$ and each retailer reports a type $\hat{v}_{i} \in \mathcal{V}_{i}$. Let $\hat{U}_{i}=\left(\hat{v}_{i}, \hat{C}_{i}\right)$ and $\hat{U}=\left[\hat{U}_{1}, \hat{U}_{2}, \ldots, \hat{U}_{I}\right]$. Then, generation dispatch $\mathbf{x}(\hat{U})$ and demand $\mathbf{d}(\hat{U})$ are identified. Each demand aggregator $i$ is charged $\tau_{i}(\hat{U})$ and each generator is compensated $\pi_{i}(\hat{U})$.

Assuming quasi-linear preferences, a direct mechanism is incentive compatible in dominant strategies (or strategy-proof) if for each demand aggregator

$$
v_{i}\left(\mathbf{d}\left(v_{i}, \hat{U}_{-i}\right)\right)-\tau_{i}\left(v_{i}, \hat{U}_{-i}\right) \geq v_{i}\left(\mathbf{d}\left(\hat{v}_{i}, \hat{U}_{-i}\right)\right)-\tau_{i}\left(\hat{v}_{i}, \hat{U}_{-i}\right), \forall \hat{v}_{i} \in \mathcal{V}_{i}, \hat{U}_{-i} \in \mathcal{U}_{-i}
$$

and for each generator

$$
\pi_{i}\left(C_{i}, \hat{U}_{-i}\right)-C_{i}\left(\mathbf{x}\left(C_{i}, \hat{U}_{-i}\right)\right) \geq \pi_{i}\left(\hat{C}_{i}, \hat{U}_{-i}\right)-C_{i}\left(\mathbf{x}\left(\hat{C}_{i}, \hat{U}_{-i}\right)\right), \forall \hat{C}_{i} \in \mathcal{C}_{i}, \hat{U}_{-i} \in \mathcal{U}_{-i}
$$

where $\hat{U}_{-i}$ (similarly $\mathcal{U}_{-i}$ ) represents the information reported by all market participants except demand aggregator (respectively, generator) located in node $i$. In other words, this says that reporting the true function $v_{i}$ (respectively, $C_{i}$ ) is optimal regardless of the announcements made by other users, say $\hat{U}_{-i}$. It is worth recalling here incentive compatibility in dominant strategies (strategy-proofness) is a stronger property than incentive compatibility 
in Bayes-Nash equilibrium strategies which is the solution concept used in Silva et al. [87] and Jofré et al. [47]. ${ }^{2}$

Direct mechanisms require complex information exchange between market participants and the market-maker. Strategic behavior induced by direct mechanisms can be quite complex (see for example, Anderson et al. [2] and Holmberg and Newbery [41] for the case of discriminatory auctions). In the next section we introduce an iterative mechanism that relies on simpler informational exchange. The basic idea is to introduce marginal adjustments to a tentative power flow solution while making sure the pricing incentives for marginal adjustment are strategy-proof. As we shall show in what follows, this allows the market-maker to identify the optimal solution to problem (5.1).

\subsection{An Iterative Mechanism}

The iterative mechanism proposed works as follows. At the beginning of iteration $t \geq 0$, each demand aggregator has a tentative schedule for consumption profile $d_{i}^{t} \geq 0$. Similarly, each generator $i$ is tentatively scheduled to deliver $x_{i}^{t}$ units. The tentative schedule is a feasible solution to problem (5.1). Each demand aggregator and each generator respectively submit information $u_{i}^{t+1}$ and $c_{i}^{t+1}$ on the value and cost respectively associated to small changes to the tentative schedule. A market-maker takes these inputs and solves for an economically efficient adjustment to the tentative schedule ensuring that the network constraints are satisfied. Then, the tentative schedules for demand and production are updated to $d_{i}^{t+1}$ and production $x_{i}^{t+1}$. The mechanism closes when a given stopping rule is met. Formally, the iterative mechanism proposed is as follows.

1. Initialization. Start with any feasible pair $\left(\mathbf{x}^{0}, \mathbf{d}^{0}\right)$.

2. Demand and Generation updates. At each round (or iteration) $t \geq 0$, each demand aggregator and each generator respectively submit information $u_{i}^{t+1} \in \mathbb{R}_{+}$and

${ }^{2}$ See Wilson [106] for a critique of a common knowledge assumption on a joint distribution for private information. 
$c_{i}^{t+1} \in \mathbb{R}_{+}$. The market maker solves

$$
\left\{\begin{aligned}
\max \sum_{i}\left(u_{i}^{t+1} d_{i}-c_{i}^{t+1} x_{i}\right) & \\
\text { s.t } & \sum_{i}\left(x_{i}-d_{i}\right) \\
-z_{\ell} \leq \sum_{i} \eta_{\ell, i}\left(x_{i}-d_{i}\right) & \leq z_{\ell} \\
\underline{x}_{i} \leq x_{i} & \leq \bar{x}_{i} \\
d_{i} & \geq 0
\end{aligned}\right.
$$

Let $\left(\hat{\mathbf{x}}^{t+1}, \hat{\mathbf{d}}^{t+1}\right)$ denote a solution to the linear program (5.2). The tentative schedules for production and consumption are updated as follows:

$$
\mathbf{x}^{t+1}=\mathbf{x}^{t}+\alpha_{t}\left(\hat{\mathbf{x}}^{t+1}-\mathbf{x}^{t}\right)
$$

and

$$
\mathbf{d}^{t+1}=\mathbf{d}^{t}+\alpha_{t}\left(\hat{\mathbf{d}}^{t+1}-\mathbf{d}^{t}\right)
$$

where $\left\{\alpha_{t}: t \geq 0\right\}$ is a sequence of stepsizes that satisfy $\alpha_{t} \in(0,1), \alpha_{t} \rightarrow 0^{+}$, and

$$
\sum_{t=1}^{\infty} \alpha_{t}=\infty, \quad \sum_{t=0}^{\infty} \alpha_{t}^{2}=M<\infty
$$

3. Incentive Payment: Each demand aggregator $i \in \mathcal{I}$ is assessed a payment of $\Delta \tau_{i}^{t+1}$ which is computed as follows

$$
\Delta \tau_{i}^{t+1}=-\sum_{k \neq i}\left(d_{k}^{t+1}-d_{k}^{t}\right) u_{k}^{t+1}+\sum_{k}\left(x_{k}^{t+1}-x_{k}^{t}\right) c_{k}^{t+1}
$$

Each generator $i \in \mathcal{I}$ is assessed a compensation of $\Delta \pi_{i}^{t+1}$ which is computed as follows

$$
\Delta \pi_{i}^{t+1}=\sum_{k}\left(d_{k}^{t+1}-d_{k}^{t}\right) u_{k}^{t+1}-\sum_{k \neq i}\left(x_{k}^{t+1}-x_{k}^{t}\right) c_{k}^{t+1}
$$

The total payment and compensation accrued after $t+1$ rounds is given by:

$$
\tau_{i}^{t+1}=\tau_{i}^{t}+\Delta \tau_{i}^{t+1}, \quad \pi_{i}^{t+1}=\pi_{i}^{t}+\Delta \pi_{i}^{t+1} .
$$


4. Activity Rule: The market-maker checks that

$$
\left(d_{i}^{\tilde{t}}-d_{i}^{t}\right) \cdot\left(u_{i}^{\tilde{t}+1}-u_{i}^{t+1}\right)<0 \quad\left(x_{i}^{\tilde{t}}-x_{i}^{t}\right) \cdot\left(c_{i}^{\tilde{t}+1}-c_{i}^{t+1}\right)>0
$$

for all $\tilde{t}<t$ and for each generator and demand aggregator located in node $i$. A participant is removed from market-clearing when this activity rule is violated.

5. Stopping Rule: If complementary slackness between the tentative schedule and Lagrange multipliers in the optimal solution to problem (5.2) is approximately verified, then STOP. Otherwise, a new iteration (steps 2 and 3) takes place.

\subsubsection{Remarks}

\section{Information Flow}

The information regarding the tentative schedule adjustments $x_{i}^{t+1}-x_{i}^{t}$ (respectively, $d_{i}^{t+1}-$ $d_{i}^{t}$ ) and associated compensation $\Delta \pi_{i}^{t+1}$ (respectively, $\Delta \tau_{i}^{t+1}$ ) are privately communicated by the market-maker to each generator (respectively demand aggregator) located in node $i$. This information flow precludes the possibility of learning by any given participant regarding the likely types of other market participants. ${ }^{3}$

\section{Pricing Rule}

The pricing rule can be seen as an implementation of Groves-Clarke pricing for small adjustments (see $[17,37])$. To gain an intuition note that not reporting the true marginal cost can only reduce a generator's marginal surplus. By reporting a higher marginal cost, the optimal solution implies the production levels of other less efficient generators will be marginally increased and there will be a marginal reduction in consumption by demand aggregators. Since the compensation is proportional to the marginal change in surplus to other generators and all demand aggregators, reporting a higher marginal cost is not in the given generator's

\footnotetext{
${ }^{3}$ The implications of strategic learning are examined in dynamic mechanism design (see Bergemann and Pavan [5]).
} 
best interest when the tentative schedule is not efficient. A similar argument can be made to show reporting a lower marginal cost is not optimal.

\section{Activity Rule}

The activity rule is equivalent to checking that the reporting strategies $\left\{u_{i}^{t+1}: t>0\right\}$ for a demand aggregator (respectively, $\left\{c_{i}^{t+1}: t>0\right\}$ for a generator) must be consistent with some concave valuation function (respectively, convex cost function). Hence, to check that the iterative design proposed is strategy-proof one needs only check that it is not optimal for any demand aggregator (respectively, generator) to report information in a manner consistent with some concave valuation function (respectively, convex cost function).

Denote by $v_{i}^{\prime}(\cdot)$ (respectively, $\left.C_{i}^{\prime}(\cdot)\right)$ the derivative function of $v_{i}$ (respectively, $C_{i}$ ). In what follows we say that demand aggregators are truthful if $u_{i}^{t+1}=v_{i}^{\prime}\left(d_{i}^{t}\right)$. Similarly, generators are truthful if $c_{i}^{t+1}=C_{i}^{\prime}\left(x_{i}^{t}\right)$.

\subsection{Analysis}

The strategy for analysis is as follows. First, assuming that demand aggregators and generators are truthful we will show convergence to optimal dispatch. Then we will show that it is optimal for every participant (demand aggregator or generator) to be truthful regardless of the reporting strategies implemented by other market participants. In what follows we assume that Assumption 6 holds.

\subsubsection{Convergence}

Consider the objective function:

$$
\mathcal{F}(\mathbf{x}, \mathbf{d}):=\sum_{i \in \mathcal{I}}\left[v_{i}\left(d_{i}\right)-C_{i}\left(x_{i}\right)\right]
$$

Lemma 3. The sequence $\left\{\mathcal{F}\left(\mathbf{x}^{t}, \mathbf{d}^{t}\right): t>0\right\}$ converges. 
Proof. By Assumption 6, $v_{i}^{\prime}(\cdot)$ and $C_{i}^{\prime}(\cdot)$ are Lipschitz continuous for all $i \in \mathcal{I}$. Hence $\mathcal{F}(\mathbf{x}, \mathbf{d})$ has Lipschitz continuous gradient, so that (see Nesterov [70])

$$
\begin{aligned}
\mathcal{F}\left(\mathbf{x}^{t+1}, \mathbf{d}^{t+1}\right) \geq \mathcal{F}\left(\mathbf{x}^{t}, \mathbf{d}^{t}\right)+\nabla_{\mathbf{d}} \mathcal{F}\left(\mathbf{x}^{t}, \mathbf{d}^{t}\right) \cdot\left(\mathbf{d}^{t+1}-\mathbf{d}^{t}\right)+\nabla_{\mathbf{x}} \mathcal{F}\left(\mathbf{x}^{t}, \mathbf{d}^{t}\right) \cdot\left(\mathbf{x}^{t+1}-\mathbf{x}^{t}\right) \\
-\frac{1}{2} L\left\|\mathbf{d}^{t+1}+\mathbf{x}^{t+1}-\mathbf{d}^{t}-\mathbf{x}^{t}\right\|^{2}
\end{aligned}
$$

for some Lipschitz constant $L>0$. Given that $\mathbf{x}^{t+1}=\mathbf{x}^{t}+\alpha_{t}\left(\hat{\mathbf{x}}^{t+1}-\mathbf{x}^{t}\right)$ and $\mathbf{d}^{t+1}=$ $\mathbf{d}^{t}+\alpha_{t}\left(\hat{\mathbf{d}}^{t+1}-\mathbf{d}^{t}\right)$, the inequality above can be rewritten as

$$
\begin{aligned}
\mathcal{F}\left(\mathbf{x}^{t+1}, \mathbf{d}^{t+1}\right) \geq \mathcal{F}\left(\mathbf{x}^{t}, \mathbf{d}^{t}\right)+\alpha_{t} \nabla_{\mathbf{d}} \mathcal{F}\left(\mathbf{x}^{t}, \mathbf{d}^{t}\right) \cdot\left(\hat{\mathbf{d}}^{t+1}-\mathbf{d}^{t}\right) & +\alpha_{t} \nabla_{\mathbf{x}} \mathcal{F}\left(\mathbf{x}^{t}, \mathbf{d}^{t}\right) \cdot\left(\hat{\mathbf{x}}^{t+1}-\mathbf{x}^{t}\right) \\
& -\frac{\alpha_{t}^{2}}{2} L\left\|\hat{\mathbf{d}}^{t+1}+\hat{\mathbf{x}}^{t+1}-\mathbf{d}^{t}-\mathbf{x}^{t}\right\|^{2} .
\end{aligned}
$$

Notice that the feasible region specified by the constraints in (5.2) is both convex and compact. Since $\hat{\mathbf{d}}^{t+1}$ and $\hat{\mathbf{x}}^{t+1}$ are feasible, $\mathbf{d}^{t+1}=\left(1-\alpha_{t}\right) \mathbf{d}^{t}+\alpha_{t} \hat{\mathbf{d}}^{t+1}$ and $\mathbf{x}^{t+1}=(1-$ $\left.\alpha_{t}\right) \mathbf{x}^{t}+\alpha_{t} \hat{\mathbf{x}}^{t+1}$, a simple induction shows that $\mathbf{d}^{t+1}$ and $\mathbf{x}^{t+1}$ are also feasible. Therefore, $\left\|\hat{\mathbf{d}}^{t+1}+\hat{\mathbf{x}}^{t+1}-\mathbf{d}^{t}-\mathbf{x}^{t}\right\|^{2} \leq D$ for some $D>0$. We have

$$
\begin{aligned}
\mathcal{F}\left(\mathbf{x}^{t+1}, \mathbf{d}^{t+1}\right) & \geq \mathcal{F}\left(\mathbf{x}^{t}, \mathbf{d}^{t}\right)+\alpha_{t} \sum_{i}\left(\hat{d}_{i}^{t+1}-d_{i}^{t}\right) v_{i}^{\prime}\left(d_{i}^{t}\right)-\alpha_{t} \sum_{i}\left(\hat{x}_{i}^{t+1}-x_{i}^{t}\right) \cdot C_{i}^{\prime}\left(x_{i}^{t}\right)-\frac{\alpha_{t}^{2}}{2} L D \\
& =\mathcal{F}\left(\mathbf{x}^{t}, \mathbf{d}^{t}\right)+\alpha_{t}\left[\sum_{i}\left(u_{i}^{t+1} \hat{d}_{i}^{t+1}-c_{i}^{t+1} \hat{x}_{i}^{t+1}\right)-\sum_{i}\left(u_{i}^{t+1} d_{i}^{t}-c_{i}^{t+1} x_{i}^{t}\right)\right]-\frac{\alpha_{t}^{2}}{2} L D \\
& \geq \mathcal{F}\left(\mathbf{x}^{t}, \mathbf{d}^{t}\right)-\frac{\alpha_{t}^{2}}{2} L D
\end{aligned}
$$

where the last inequality is due to the fact that $\left(\hat{\mathbf{x}}^{t+1}, \hat{\mathbf{d}}^{t+1}\right)$ is the solution to (5.2) for which $\left(\mathbf{x}^{t}, \mathbf{d}^{t}\right)$ is feasible.

Since the sequence $\left(\mathbf{x}^{t}, \mathbf{d}^{t}\right)$ is bounded and $\mathcal{F}(\mathbf{x}, \mathbf{d})$ is Lipschitz continuous, $\mathcal{F}\left(\mathbf{x}^{t}, \mathbf{d}^{t}\right)$ is also bounded. Now assume by contradiction that $\left\{\mathcal{F}\left(\mathbf{x}^{t}, \mathbf{d}^{t}\right): t>0\right\}$ does not converge. Then $\limsup \mathcal{F}\left(\mathbf{x}^{t}, \mathbf{d}^{t}\right)>\liminf \mathcal{F}\left(\mathbf{x}^{t}, \mathbf{d}^{t}\right)$. There exists $\overline{\mathcal{F}}>\underline{\mathcal{F}}$ such that both $\mathcal{F}\left(\mathbf{x}^{t}, \mathbf{d}^{t}\right)>\overline{\mathcal{F}}$ and $\mathcal{F}\left(\mathbf{x}^{t}, \mathbf{d}^{t}\right)<\underline{\mathcal{F}}$ infinitely often. But $\mathcal{F}\left(\mathbf{x}^{t+1}, \mathbf{d}^{t+1}\right)-\mathcal{F}\left(\mathbf{x}^{t}, \mathbf{d}^{t}\right) \geq-\left(\alpha_{t}^{2} / 2\right) L D$ and $\sum_{t} \alpha_{t}^{2}<\infty$ implies that such up-crossings and down-crossings cannot take place infinitely often. We conclude that the sequence $\left\{\mathcal{F}\left(\mathbf{x}^{t}, \mathbf{d}^{t}\right): t>0\right\}$ converges. 
Theorem 6. Assuming that generators and demand aggregators are truthful, $\left(\mathbf{x}^{t}, \mathbf{d}^{t}\right)$ converges to the unique solution of problem (5.1).

Proof. See Appendix A.

\subsubsection{Incentive Compatibility}

Unlike direct mechanisms, dynamic strategic behavior must be taken into account when analyzing the incentive compatibility properties of an iterative mechanism. As mentioned before, the proposed design features bilateral information exchange regarding the tentative dispatch the payment or compensation for adjustments between market-maker and each market participant. This information architecture precludes the possibility of statistical learning by any given participant regarding the likely types of other market participants. Another important design feature corresponds to the activity rule. This test ensures that the reporting strategies by a generator (respectively, demand aggregator) must be consistent over time with some convex cost function (respectively, concave valuation function). These design features play an important role in ensuring the iterative mechanism is approximately strategy-proof. That is to say, truthful reporting is arbitrarily close to being an optimal strategy regardless of the reporting strategies of other market participants.

Theorem 7. Truthful reporting is a $\delta^{M}>0$ dominant strategy where $\delta^{M}=\frac{1}{2} L D M$.

Proof. We start by showing that truthful reporting is a $\delta^{M}$-Nash equilibrium. Then we show that truthful reporting is $\delta^{M}$-optimal regardless of the reporting strategies of other market participants. We start with estimating the total payment for demand aggregator $i$ (respectively, generator $i$ ) under truthful reporting assuming all other demand aggregators and generators are truthful. Referring to (5.5), after $T$ rounds the total payment for demand aggregator $i$ is

$$
\tau_{i}^{T}=\tau_{i}^{0}-\sum_{t=0}^{T-1} \sum_{k \neq i}\left(d_{k}^{t+1}-d_{k}^{t}\right) u_{k}^{t+1}+\sum_{t=0}^{T-1} \sum_{k}\left(x_{k}^{t+1}-x_{k}^{t}\right) c_{k}^{t+1} .
$$


On one hand, notice that for all $k \in \mathcal{I}$,

$$
v_{k}\left(d_{k}^{t+1}\right)-v_{k}\left(d_{k}^{t}\right) \leq v_{k}^{\prime}\left(d_{k}^{t}\right)\left(d_{k}^{t+1}-d_{k}^{t}\right)=\left(d_{k}^{t+1}-d_{k}^{t}\right) u_{k}^{t+1}
$$

by concavity of $v_{k}$ and the truthful reporting assumption, and

$$
C_{k}\left(x_{k}^{t+1}\right)-C_{k}\left(x_{k}^{t}\right) \geq\left(x_{k}^{t+1}-x_{k}^{t}\right) C_{k}^{\prime}\left(x_{k}^{t}\right)=\left(x_{k}^{t+1}-x_{k}^{t}\right) c_{k}^{t+1}
$$

by convexity of $C_{k}$ and the truthful reporting assumption. We have

$$
\begin{aligned}
\tau_{i}^{T} & \leq \tau_{i}^{0}-\sum_{t=0}^{T-1} \sum_{k \neq i}\left(v_{k}\left(d_{k}^{t+1}\right)-v_{k}\left(d_{k}^{t}\right)\right)+\sum_{t=0}^{T-1} \sum_{k}\left(C_{k}\left(x_{k}^{t+1}\right)-C_{k}\left(x_{k}^{t}\right)\right) \\
& =\tau_{i}^{0}-\left(\sum_{k \neq i} v_{k}\left(d_{k}^{T}\right)-\sum_{k} C_{k}\left(x_{k}^{T}\right)\right)+\sum_{k \neq i} v_{k}\left(d_{k}^{0}\right)-\sum_{k} C_{k}\left(x_{k}^{0}\right) .
\end{aligned}
$$

On the other hand,

$$
\begin{aligned}
\tau_{i}^{T}= & \tau_{i}^{0}-\sum_{t=0}^{T-1} \sum_{k}\left(d_{k}^{t+1}-d_{k}^{t}\right) u_{k}^{t+1}+\sum_{t=0}^{T-1} \sum_{k}\left(x_{k}^{t+1}-x_{k}^{t}\right) c_{k}^{t+1}+\sum_{t=0}^{T-1}\left(d_{i}^{t+1}-d_{i}^{t}\right) u_{i}^{t+1} \\
\geq & \tau_{i}^{0}-\sum_{t=0}^{T-1} \sum_{k}\left(v_{k}\left(d_{k}^{t+1}\right)-v_{k}\left(d_{k}^{t}\right)\right)+\sum_{t=0}^{T-1} \sum_{k}\left(C_{k}\left(x_{k}^{t+1}\right)-C_{k}\left(x_{k}^{t}\right)\right) \\
& -\sum_{t=0}^{T-1} \frac{1}{2} L\left\|\mathbf{d}^{t+1}+\mathbf{x}^{t+1}-\mathbf{d}^{t}-\mathbf{x}^{t}\right\|^{2}+\sum_{t=0}^{T-1}\left(v_{i}\left(d_{i}^{t+1}\right)-v_{i}\left(d_{i}^{t}\right)\right) \\
= & \tau_{i}^{0}-\left(\sum_{k \neq i} v_{k}\left(d_{k}^{T}\right)-\sum_{k} C_{k}\left(x_{k}^{T}\right)\right)+\sum_{k \neq i} v_{k}\left(d_{k}^{0}\right)-\sum_{k} C_{k}\left(x_{k}^{0}\right) \\
& -\sum_{t=0}^{T-1} \frac{1}{2} L\left\|\mathbf{d}^{t+1}+\mathbf{x}^{t+1}-\mathbf{d}^{t}-\mathbf{x}^{t}\right\|^{2},
\end{aligned}
$$

where the inequality comes from the Lipschitz condition. Recalling that $\mathbf{x}^{t+1}-\mathbf{x}^{t}=\alpha_{t}\left(\hat{\mathbf{x}}^{t+1}-\right.$ $\left.\mathbf{x}^{t}\right)$ and $\mathbf{d}^{t+1}-\mathbf{d}^{t}=\alpha_{t}\left(\hat{\mathbf{d}}^{t+1}-\mathbf{d}^{t}\right)$,

$$
\tau_{i}^{T} \geq \tau_{i}^{0}-\left(\sum_{k \neq i} v_{k}\left(d_{k}^{T}\right)-\sum_{k} C_{k}\left(x_{k}^{T}\right)\right)+\sum_{k \neq i} v_{k}\left(d_{k}^{0}\right)-\sum_{k} C_{k}\left(x_{k}^{0}\right)-\frac{1}{2} L D M .
$$

Analogously, $\pi_{i}^{T}$ satisfies

$$
\pi_{i}^{T} \geq \pi_{i}^{0}+\sum_{k} v_{k}\left(d_{k}^{T}\right)-\sum_{k \neq i} C_{k}\left(x_{k}^{T}\right)-\sum_{k} v_{k}\left(d_{k}^{0}\right)+\sum_{k \neq i} C_{k}\left(x_{k}^{0}\right)
$$


and

$$
\pi_{i}^{T} \leq \pi_{i}^{0}+\sum_{k} v_{k}\left(d_{k}^{T}\right)-\sum_{k \neq i} C_{k}\left(x_{k}^{T}\right)-\sum_{k} v_{k}\left(d_{k}^{0}\right)+\sum_{k \neq i} C_{k}\left(x_{k}^{0}\right)+\frac{1}{2} L D M .
$$

Hence the net surplus for demand aggregator $i$ satisfies

$$
\begin{aligned}
v_{i}\left(d_{i}^{T}\right)-\tau_{i}^{T} \in\left[\mathcal{F}\left(\mathbf{x}^{T}, \mathbf{d}^{T}\right)-\mathcal{F}\left(\mathbf{x}^{0}, \mathbf{d}^{0}\right)\right. & +v_{i}\left(d_{i}^{0}\right)-\tau_{i}^{0}, \\
& \left.\mathcal{F}\left(\mathbf{x}^{T}, \mathbf{d}^{T}\right)-\mathcal{F}\left(\mathbf{x}^{0}, \mathbf{d}^{0}\right)+v_{i}\left(d_{i}^{0}\right)-\tau_{i}^{0}+\frac{1}{2} L D M\right] .
\end{aligned}
$$

In the limit as $T \rightarrow \infty$,

$$
v_{i}\left(d_{i}^{T}\right)-\tau_{i}^{T} \geq \mathcal{F}\left(\mathbf{x}^{*}, \mathbf{d}^{*}\right)-\mathcal{F}\left(\mathbf{x}^{0}, \mathbf{d}^{0}\right)+v_{i}\left(d_{i}^{0}\right)-\tau_{i}^{0} .
$$

Now assume that demand aggregator $i$ engages in untruthful reporting consistently with some convex value function $\tilde{v}_{i}$, i.e. $u_{i}^{t+1}=\tilde{v}_{i}^{\prime}\left(d_{i}^{t}\right)$. We can invoke Theorem (6) to show that this untruthful behavior leads to the optimal solution of problem (5.1) where in the function $v_{i}$ is replaced by $\tilde{v}_{i}$. Let $\left(\tilde{\mathbf{x}}^{*}, \tilde{\mathbf{d}}^{*}\right)$ be the optimal solution. Then,

$$
\begin{aligned}
v_{i}\left(d_{i}^{T}\right)-\tau_{i}^{T} & \geq \mathcal{F}\left(\mathbf{x}^{*}, \mathbf{d}^{*}\right)-\mathcal{F}\left(\mathbf{x}^{0}, \mathbf{d}^{0}\right)+v_{i}\left(d_{i}^{0}\right)-\tau_{i}^{0} \\
& \geq \mathcal{F}\left(\tilde{\mathbf{x}}^{*}, \tilde{\mathbf{d}}^{*}\right)-\mathcal{F}\left(\mathbf{x}^{0}, \mathbf{d}^{0}\right)+v_{i}\left(d_{i}^{0}\right)-\tau_{i}^{0} \\
& \geq v_{i}\left(\tilde{d}_{i}^{T}\right)-\tilde{\tau}_{i}^{T}-\frac{1}{2} L D M,
\end{aligned}
$$

where $\tilde{\tau}_{i}^{T}$ is defined correspondingly. Therefore, it is $\delta^{M}$-optimal for demand aggregator $i$ to be truthful when all other market participants are truthful. Similarly, to see that it is also $\delta^{M}$-optimal for generator $i$ to be truthful (when all other participants are truthful) note that the net surplus for generator $i$ satisfies

$$
\begin{aligned}
\pi_{i}^{T}-C_{i}\left(x_{i}^{T}\right) \in\left[\mathcal{F}\left(\mathbf{x}^{T}, \mathbf{d}^{T}\right)-\mathcal{F}\left(\mathbf{x}^{0}, \mathbf{d}^{0}\right)\right. & -C_{i}\left(x_{i}^{0}\right)+\pi_{i}^{0}, \\
& \left.\mathcal{F}\left(\mathbf{x}^{T}, \mathbf{d}^{T}\right)-\mathcal{F}\left(\mathbf{x}^{0}, \mathbf{d}^{0}\right)-C_{i}\left(x_{i}^{0}\right)+\pi_{i}^{0}+\frac{1}{2} L D M\right] .
\end{aligned}
$$

As $T \rightarrow \infty$,

$$
\begin{aligned}
\pi_{i}^{T}-C_{i}\left(x_{i}^{T}\right) & \geq \mathcal{F}\left(\mathbf{x}^{*}, \mathbf{d}^{*}\right)-\mathcal{F}\left(\mathbf{x}^{0}, \mathbf{d}^{0}\right)-C_{i}\left(x_{i}^{0}\right)+\pi_{i}^{0} \\
& \geq \mathcal{F}\left(\tilde{\mathbf{x}}^{*}, \tilde{\mathbf{d}}^{*}\right)-\mathcal{F}\left(\mathbf{x}^{0}, \mathbf{d}^{0}\right)-C_{i}\left(x_{i}^{0}\right)+\pi_{i}^{0} \\
& \geq \tilde{\pi}_{i}^{T}-C_{i}\left(\tilde{d}_{i}^{T}\right)-\frac{1}{2} L D M .
\end{aligned}
$$


Now consider the case in which market participants other than demand aggregator $i$ report consistently according to concave value functions $\tilde{v}_{k}, k \neq i$ and convex functions $\tilde{c}_{i}$, i.e., $u_{k}^{t+1}=\tilde{v}_{k}^{\prime}\left(d_{k}^{t}\right)$ and $c_{i}^{t+1}=\tilde{C}_{i}^{\prime}\left(x_{i}^{t}\right)$. If demand aggregator $i$ is truthful then Theorem (6) ensures that $\left(\mathbf{x}^{t}, \mathbf{d}^{t}\right) \rightarrow(\hat{\mathbf{x}}, \hat{\mathbf{d}})$ where this limit point is the solution to problem (5.1) with the value functions for all demand aggregators $k \neq i$ and generators are substituted by $\tilde{v}_{k}$ and $\tilde{c}_{i}$. Similarly when demand aggregator $i$ consistently reports according to some concave value function $\tilde{v}_{i}$ Theorem (6) ensures that $\left(\mathbf{x}^{t}, \mathbf{d}^{t}\right) \rightarrow(\tilde{\mathbf{x}}, \tilde{\mathbf{d}})$ where this limit point is the solution to problem (5.1) with the value and cost functions $\tilde{v}_{i}$ and $\tilde{c}_{i}$. Let

$$
\mathcal{F}_{i}(\mathbf{x}, \mathbf{d}):=v_{i}\left(d_{i}\right)+\sum_{k \neq i} \tilde{v}_{k}\left(d_{k}\right)-\sum_{i \in \mathcal{I}} \tilde{C}_{i}\left(x_{i}\right)
$$

Then,

$$
\begin{aligned}
v_{i}\left(\hat{d}_{i}^{T}\right)-\hat{\tau}_{i}^{T} & \geq \mathcal{F}_{i}(\hat{\mathbf{x}}, \hat{\mathbf{d}})-\mathcal{F}_{i}\left(\mathbf{x}^{0}, \mathbf{d}^{0}\right)+v_{i}\left(d_{i}^{0}\right)-\tau_{i}^{0} \\
& \geq \mathcal{F}_{i}(\tilde{\mathbf{x}}, \tilde{\mathbf{d}})-\mathcal{F}_{i}\left(\mathbf{x}^{0}, \mathbf{d}^{0}\right)+v_{i}\left(d_{i}^{0}\right)-\tau_{i}^{0} \\
& \geq v_{i}\left(\tilde{d}_{i}^{T}\right)-\tilde{\tau}_{i}^{T}-\frac{1}{2} L D M
\end{aligned}
$$

Therefore, truthful reporting is a $\delta^{M}$-dominant strategy for demand aggregator $i$. A similar argument can be made for generator $i$.

Note that $\delta^{M}$ can be made arbitrarily small by ensuring adjustments at each iteration are small though the condition $\sum_{t} \alpha_{t} \rightarrow \infty$ must be satisfied. This indicates that there is an inverse relationship between convergence speed and the strength of the incentive compatibility property. Faster convergence requires not so small adjustments (at least early in the process). Without so small adjustments, the pricing rule only provides a rough approximation to the change in surplus so that the incentive compatibility property is necessarily weakened.

\subsubsection{Participation Constraints and Budget Balance}

Let $\underline{\mathcal{F}}$ denote a lower bound of $\mathcal{F}\left(\mathbf{x}^{*}, \mathbf{d}^{*}\right)$ such that $\underline{\mathcal{F}} \geq \frac{2 I-1}{2 I} \mathcal{F}\left(\mathbf{x}^{*}, \mathbf{d}^{*}\right)$. Let

$$
\tau_{i}^{0}=\underline{\mathcal{F}}, \quad \pi_{i}^{0}=-\underline{\mathcal{F}} .
$$


Assume that $\left(\mathbf{x}^{0}, \mathbf{d}^{0}\right)=(0,0)$ and $v_{i}(0)=C_{i}(0)=0$ so that $\mathcal{F}\left(\mathbf{x}^{0}, \mathbf{d}^{0}\right)=0$. If $\delta^{M} \simeq 0$ it follows from the inequalities obtained in the proof of Theorem 7 that:

$$
v_{i}\left(d_{i}^{T}\right)-\tau_{i}^{T} \simeq \mathcal{F}\left(\mathbf{x}^{*}, \mathbf{d}^{*}\right)-\tau_{i}^{0}>0, \quad \pi_{i}^{T}-C_{i}\left(x_{i}^{T}\right) \simeq \mathcal{F}\left(\mathbf{x}^{*}, \mathbf{d}^{*}\right)+\pi_{i}^{0}>0 .
$$

It can be seen that market participants share equally (approximately) the net surplus from trade. This implies that individual rationality constraints are met (assuming an outside option yielding zero surplus). To see that the mechanism is weakly budget balanced (approximately) note that

$$
\tau_{i}^{T} \simeq \tau_{i}^{0}-\left(\sum_{k \neq i} v_{k}\left(d_{k}^{T}\right)-\sum_{k} C_{k}\left(x_{k}^{T}\right)\right), \quad \pi_{i}^{T} \simeq \pi_{i}^{0}+\sum_{k} v_{k}\left(d_{k}^{T}\right)-\sum_{k \neq i} C_{k}\left(x_{k}^{T}\right)
$$

The margin for the market maker is

$$
\sum_{i \in \mathcal{I}}\left(\tau_{i}^{T}-\pi_{i}^{T}\right) \simeq \sum_{i \in \mathcal{I}}\left(\tau_{i}^{0}-\pi_{i}^{0}\right)-(2 I-1) \mathcal{F}\left(\mathbf{x}^{*}, \mathbf{d}^{*}\right)=2 I\left(\underline{\mathcal{F}}-\frac{2 I-1}{2 I} \mathcal{F}\left(\mathbf{x}^{*}, \mathbf{d}^{*}\right)\right) \geq 0
$$

\subsubsection{Variations on a Theme}

\section{Other Iterative Mechanisms}

In the iterative mechanism proposed above, there is an inverse relationship between convergence speed (a function of the schedule $\left\{\alpha_{t}: t>0\right\}$ ) and the strength of the incentive compatibility property (a function of $M=\sum_{t} \alpha_{t}^{2}$ ). Faster convergence requires not so small adjustments (at least early in the process). However, when the adjustments are not so small the pricing rule only provides a rough approximation to the change in surplus so that the incentive compatibility property is necessarily weakened. A more sophisticated adjustment rule involving a smoothening term may provide a better tradeoff at the expense of additional computational effort. Specifically, Step 2 (Demand and Generation Updates) may be replaced by the following:

$2^{\prime}$ Demand and Generation updates. Each demand aggregator and each generator 
respectively submit bids $u_{i}^{t+1}$ and $c_{i}^{t+1}$. The market maker solves

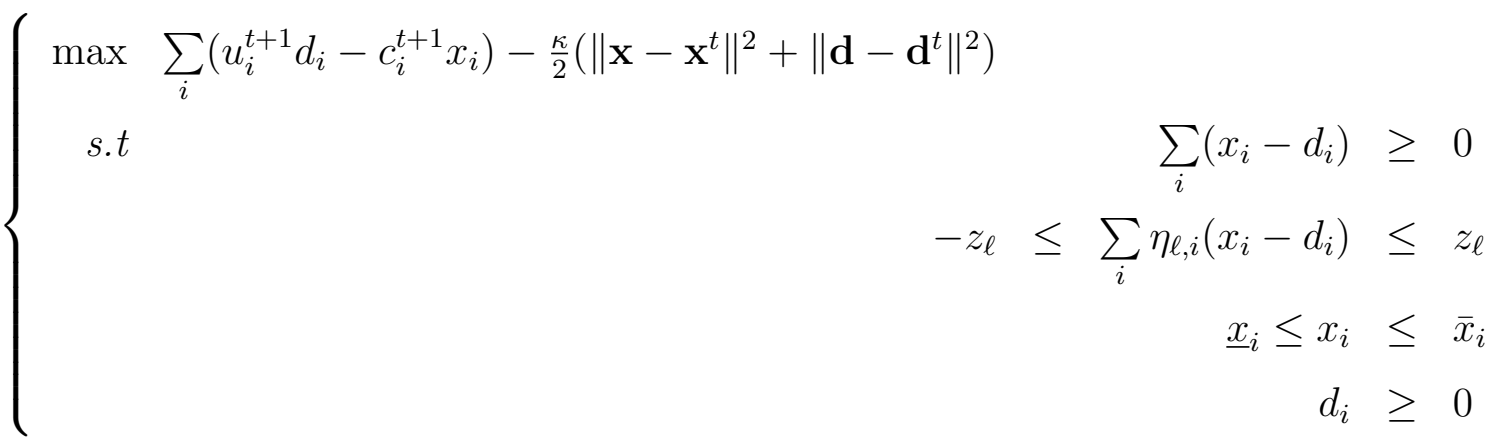

Let $\left(\hat{\mathbf{x}}^{t+1}, \hat{\mathbf{d}}^{t+1}\right)$ be a solution this problem. The tentative schedules for production and consumption are updated as follows:

$$
\mathbf{x}^{t+1}=\mathbf{x}^{t}+\alpha\left(\hat{\mathbf{x}}^{t+1}-\mathbf{x}^{t}\right) \quad \mathbf{d}^{t+1}=\mathbf{d}^{t}+\alpha\left(\hat{\mathbf{d}}^{t+1}-\mathbf{d}^{t}\right)
$$

where $\alpha>0$ is a fixed stepsize and $\kappa>0$.

In comparison, at each iteration according to Step $2^{\prime}$ a quadratic program must be solved (as opposed to a linear program in Step 2). Also, the step size is fixed which enables faster convergence. Indeed, for the strongly convex and Lipschitz differentiable case, this variation to the basic iterative mechanism may be shown to exhibit finite convergence guarantees (see Nesterov [70]). However, such result critically relies on having complete information on the Lipschitz constants for the cost and valuation functions, so as to properly tune the smoothening parameter $\kappa>0$.

\section{AC Power Flow}

The network constraints used in Section 5.2.1 are based upon a DC power flow model which ignores reactive power and voltage stability concerns. In general, linearized versions of power flow equations are not necessarily suitable for operational security assessment wherein nonlinear effects may play an important role. The feasibility region for AC power flow is non-convex (see Lesieutre et al. [55]). However, in a recent series of papers, several authors have developed computationally tractable approximations of the power flow feasibility region with constraints in terms of ellipsoids in injection space (see for example Saric and 
Stankovic [84], Dvijotham and Turitsyn [24]). The properties of the iterative mechanism design proposed are not dependent upon the closed-form representation of the constraints in the constrained economic dispatch problem (5.1). Rather, the key assumption is convexity. Thus, the iterative mechanism may potentially be suitably adjusted for use in conjunction with convex approximations to AC power flow. This is a subject of future research.

\subsection{Iterative Mechanism for Stochastic Market Clear- ing}

In this section, we analyze an iterative mechanism for stochastic market clearing. This is an important feature as the share of renewable (intermittent) capacity in most electricity markets around the world is increasing at a steady pace and ex-post adjustments to market dispatch are the norm. Recall, that in a direct mechanism for stochastic market clearing, the market-maker selects both a nominal dispatch and (ex-post) dispatch adjustments based upon the reports by market participants regarding their cost and valuation structure (including the cost of adjustments). Under uncertainty it is exceedingly difficult for generators to estimate their adjustment costs which may vary significantly depending upon the nominal dispatch. We shall show that the iterative mechanism allows for estimation error by market participants.

\subsubsection{Preliminaries}

As in Section 5.2.1, we assume that there is a single demand aggregator at each node $i \in \mathcal{I}$ in the network. Denote by $d_{i}$ node $i$ 's demand and $v_{i}(d)$ its valuation for consumption level $d \geq 0$. There is at most one generator per node with total $\operatorname{cost} C_{i}(x)$ for a production level $x \geq 0$. Let $\bar{C}_{i}(\Delta x, x)$ denote the cost associated to adjusting production in short notice to $x+\Delta x$ and $\Delta x \in \mathbb{R}$. Suppose that for each node $i \in \mathcal{I}$, there is a stochastic amount of power generated $\mu_{i}-\omega_{i}$. Here $\mu_{i}$ is constant, assumed known from the forecast, and $\omega_{i}$ is

a zero mean independent random variable with known distribution. Let $\Omega:=\sum_{i} \omega_{i}$. Since 
the power injections at each node are fluctuating, we use an affine control scheme (as in Bienstock et al. [6]) to ensure that generation is equal to demand at all times within the time window of interest. We assume that all generators respond to input fluctuations in a proportional way (affine control), however, with possibly different proportionality coefficients $\gamma_{i}$ satisfying $\sum_{i \in \mathcal{I}} \gamma_{i}=1$. We leave these coefficients to the optimization step to decide the optimal value.

\section{Chance Constraints}

The affine control scheme creates the possibility of power overflow on certain lines and requiring a generator to produce power beyond its limits. With large scale penetration from renewables (e.g. wind, solar) these events may be likely. However, the probability of occurrence can be restricted to arbitrarily small value as in Bienstock et al. [6]. Formally, we consider the following constraints:

$$
\left\{\begin{aligned}
\sum_{i}\left(x_{i}+\mu_{i}-d_{i}\right) & \geq 0 \quad(a) \\
\mathbb{P}\left(\sum_{i} \eta_{\ell, i}\left(x_{i}+\mu_{i}-\omega_{i}+\gamma_{i} \Omega-d_{i}\right)>z_{\ell}\right) & \leq \epsilon_{\ell}\left(b_{1}\right) \\
\mathbb{P}\left(\sum_{i} \eta_{\ell, i}\left(x_{i}+\mu_{i}-\omega_{i}+\gamma_{i} \Omega-d_{i}\right)<-z_{\ell}\right) & \leq \epsilon_{\ell}\left(b_{2}\right) \\
\mathbb{P}\left(x_{i}+\gamma_{i} \Omega>\bar{x}_{i}\right) & \leq \epsilon_{i}\left(c_{1}\right) \\
\mathbb{P}\left(x_{i}+\gamma_{i} \Omega<\underline{x}_{i}\right) & \leq \epsilon_{i}\left(c_{2}\right) \\
\mathbb{P}\left(\gamma_{i} \Omega>\bar{r}_{i}\right) & \leq \epsilon_{i r}\left(d_{1}\right) \\
\mathbb{P}\left(\gamma_{i} \Omega<-\bar{r}_{i}\right) & \leq \epsilon_{i r}\left(d_{2}\right) \\
\sum_{i} \gamma_{i} & =1 \\
\gamma_{i} & \geq 0 \\
d_{i} & \geq 0
\end{aligned}\right.
$$

Here $\left(b_{1}\right)-\left(d_{2}\right)$ are chance constraints, where $\mathbb{P}(\cdot)$ denotes the probability function, and $\epsilon_{\ell}, \epsilon_{i}, \epsilon_{i r}$ are small positive numbers. The parameter $\bar{r}_{i}$ is the limit for short-run adjustments for generator $i$.

We show below that $\left(b_{1}\right)-\left(d_{2}\right)$ can be reformulated into deterministic forms. First, 
consider $\left(b_{1}\right)$ and $\left(b_{2}\right)$. Given that

$$
\sum_{i} \eta_{\ell, i}\left(x_{i}+\mu_{i}-\omega_{i}+\gamma_{i} \Omega-d_{i}\right)=\sum_{i} \eta_{\ell, i}\left(x_{i}+\mu_{i}-\omega_{i}-d_{i}\right)+\sum_{i} \eta_{\ell, i} \gamma_{i} \Omega,
$$

We can rewrite $\left(b_{1}\right)$ as

$$
\begin{array}{r}
\mathbb{P}\left(\sum_{i} \eta_{\ell, i}\left(x_{i}+\mu_{i}-\omega_{i}+\gamma_{i} \Omega-d_{i}\right)>z_{\ell}\right)=\mathbb{P}\left(\sum_{i} \eta_{\ell, i} \gamma_{i}>\frac{z_{\ell}-\sum_{i} \eta_{\ell, i}\left(x_{i}+\mu_{i}-\omega_{i}-d_{i}\right)}{\Omega}\right) \\
\leq \epsilon_{\ell} .
\end{array}
$$

Denote the distribution function of $\left(z_{\ell}-\sum_{i} \eta_{\ell, i}\left(x_{i}+\mu_{i}-\omega_{i}-d_{i}\right)\right) / \Omega$ by $\bar{F}_{\ell}$. Thus, constraint $\left(b_{1}\right)$ is equivalent to

$$
\sum_{i} \eta_{\ell, i} \gamma_{i} \leq \bar{F}_{\ell}^{-1}\left(\epsilon_{\ell}\right)
$$

Similarly, $\left(b_{2}\right)$ yields

$$
\sum_{i} \eta_{\ell, i} \gamma_{i} \geq \underline{F}_{\ell}^{-1}\left(1-\epsilon_{\ell}\right)
$$

where $\underline{F}_{\ell}$ is the distribution function of $\left(-z_{\ell}-\sum_{i} \eta_{\ell, i}\left(x_{i}+\mu_{i}-\omega_{i}-d_{i}\right)\right) / \Omega$. By $\left(c_{1}\right)$ and $\left(c_{2}\right)$,

$$
\begin{aligned}
& \mathbb{P}\left(x_{i}+\gamma_{i} \Omega>\bar{x}_{i}\right)=\mathbb{P}\left(\Omega>\frac{\bar{x}_{i}-x_{i}}{\gamma_{i}}\right) \leq \epsilon_{i}, \\
& \mathbb{P}\left(x_{i}+\gamma_{i} \Omega<\bar{x}_{i}\right)=\mathbb{P}\left(\Omega<\frac{\underline{x}_{i}-x_{i}}{\gamma_{i}}\right) \leq \epsilon_{i} .
\end{aligned}
$$

Let $F$ be the distribution function of $\Omega$. We can write $\left(c_{1}\right)$ and $\left(c_{2}\right)$ as:

$$
\frac{\bar{x}_{i}-x_{i}}{\gamma_{i}} \geq F^{-1}\left(1-\epsilon_{i}\right), \quad \underline{\underline{x_{i}-x_{i}}} \leq F^{-1}\left(\epsilon_{i}\right),
$$

which is equivalent to

$$
x_{i}+F^{-1}\left(1-\epsilon_{i}\right) \gamma_{i} \geq \bar{x}_{i}, \quad x_{i}+F^{-1}\left(\epsilon_{i}\right) \gamma_{i} \geq \underline{x}_{i} .
$$

Finally, note that $\left(d_{1}\right)$ and $\left(d_{2}\right)$ give

$$
\mathbb{P}\left(\gamma_{i} \Omega>\bar{r}_{i}\right)=\mathbb{P}\left(\Omega>\frac{\bar{r}_{i}}{\gamma_{i}}\right) \leq \epsilon_{i r}, \quad \mathbb{P}\left(\gamma_{i} \Omega<-\bar{r}_{i}\right)=\mathbb{P}\left(\Omega<-\frac{\bar{r}_{i}}{\gamma_{i}}\right) \leq \epsilon_{i r},
$$


which is equivalent to

$$
\frac{\bar{r}_{i}}{\gamma_{i}} \geq F^{-1}\left(1-\epsilon_{i r}\right), \quad-\frac{\bar{r}_{i}}{\gamma_{i}} \leq F^{-1}\left(\epsilon_{i r}\right),
$$

or

$$
F^{-1}\left(1-\epsilon_{i r}\right) \gamma_{i} \leq \bar{r}_{i}, \quad F^{-1}\left(\epsilon_{i r}\right) \gamma_{i} \geq-\bar{r}_{i}
$$

\section{Chance Constrained Economic Dispatch}

Node $i$ 's expected cost associated with adjustments is given by $\mathbb{E}_{\Omega}\left[\bar{C}_{i}\left(\gamma_{i} \Omega, x_{i}\right)\right]$, where $\mathbb{E}(\cdot)$ denotes the function of expected value. Hence the optimal economic dispatch is the solution to:

$$
\max _{(\mathbf{x}, \mathbf{d}, \gamma)} \sum_{i}\left[v_{i}\left(d_{i}\right)-C_{i}\left(x_{i}\right)\right]-\sum_{i} \mathbb{E}_{\Omega}\left[\bar{C}_{i}\left(\gamma_{i} \Omega, x_{i}\right)\right]
$$

with constraints

$$
\left\{\begin{array}{rlr}
\sum_{i}\left(x_{i}+\mu_{i}-d_{i}\right) & \geq 0 & (a) \\
\sum_{i} \eta_{\ell, i} \gamma_{i} & \leq \bar{F}_{\ell}^{-1}\left(\epsilon_{\ell}\right) & \left(b_{1}\right) \\
\sum_{i} \eta_{\ell, i} \gamma_{i} & \geq \underline{F}_{\ell}^{-1}\left(1-\epsilon_{\ell}\right) & \left(b_{2}\right) \\
x_{i}+F^{-1}\left(1-\epsilon_{i}\right) \gamma_{i} & \geq \bar{x}_{i} & \left(c_{1}\right) \\
x_{i}+F^{-1}\left(\epsilon_{i}\right) \gamma_{i} & \geq \underline{x}_{i} & \left(c_{2}\right) \\
F^{-1}\left(1-\epsilon_{i r}\right) \gamma_{i} & \leq \bar{r}_{i} \\
F^{-1}\left(\epsilon_{i r}\right) \gamma_{i} & \geq-\bar{r}_{i} & \left(d_{1}\right) \\
\sum_{i} \gamma_{i} & =1 & \left(d_{2}\right) \\
\gamma_{i} & \geq 0 & (e) \\
d_{i} & \geq 0 & (f)
\end{array}\right.
$$

\subsubsection{Iterative Mechanism Design}

The constrained economic dispatch problem in (5.12) and (5.13) can not be solved by a benevolent intermediator such as an independent system operator because the information on $v_{i}, C_{i}$ and $\bar{C}_{i}$ is private to demand aggregators and generators. In what follows we will 
present and analyze a mechanism that guarantees market participants (demand aggregators and generators) truthfully interact with a benevolent system operator in order to identify the optimal solution to problem (5.12).

Let $\mathbf{x}=\left[x_{1}, x_{2}, \ldots, x_{I}\right], \gamma=\left[\gamma_{1}, \gamma_{2}, \ldots, \gamma_{I}\right]$ and $\mathbf{d}=\left[d_{1}, d_{2}, \ldots, d_{I}\right]$. The iterative mechanism proposed is as follows.

1. Initialization. Start with any feasible pair $\left(\mathbf{x}^{0}, \gamma^{0}, \mathbf{d}^{0}\right)$.

2. Schedule and Price updates. At each round (or iteration) $t \geq 0$ :

(a) Price update: Each demand aggregator submits bids $u_{i}^{t+1}$, and each generator submits $c_{i}^{t+1}$ and $\bar{c}_{i}^{t+1}$. The market maker solves

$$
\max _{(\mathbf{x}, \gamma, \mathbf{d})} \sum_{i}\left(u_{i}^{t+1} d_{i}-c_{i}^{t+1} x_{i}-\bar{c}_{i}^{t+1} \gamma_{i}\right)
$$

subject to constraints (5.13).

(b) Demand and Generation updates: Let $\left(\hat{\mathbf{x}}^{t+1}, \hat{\gamma}^{t+1}, \hat{\mathbf{d}}^{t+1}\right)$ denote the optimal solution to (5.14). The tentative schedules are updated as follows:

$$
\begin{aligned}
\mathbf{x}^{t+1} & =\mathbf{x}^{t}+\alpha_{t}\left(\hat{\mathbf{x}}^{t+1}-\mathbf{x}^{t}\right) \\
\gamma^{t+1} & =\gamma^{t}+\alpha_{t}\left(\hat{\gamma}^{t+1}-\gamma^{t}\right) \\
\mathbf{d}^{t+1} & =\mathbf{d}^{t}+\alpha_{t}\left(\hat{\mathbf{d}}^{t+1}-\mathbf{d}^{t}\right)
\end{aligned}
$$

where $\left\{\alpha_{t}: t \geq 0\right\}$ is a sequence of stepsizes that satisfy $\alpha_{t} \in(0,1), \alpha_{t} \rightarrow 0^{+}$and

$$
\sum_{t=1}^{\infty} \alpha_{t}=\infty, \quad \sum_{t=0}^{\infty} \alpha_{t}^{2}<\infty
$$

3. Incentive Payment: Each demand aggregator $i \in \mathcal{I}$ is assessed a payment of $\Delta \tau_{i}^{t+1}$ which is computed as follows

$$
\Delta \tau_{i}^{t+1}=-\sum_{k \neq i}\left(d_{k}^{t+1}-d_{k}^{t}\right) u_{k}^{t+1}+\sum_{k}\left(x_{k}^{t+1}-x_{k}^{t}\right) c_{k}^{t+1}+\sum_{k}\left(\gamma_{k}^{t+1}-\gamma_{k}^{t}\right) \bar{c}_{k}^{t+1}
$$

Each generator $i \in \mathcal{I}$ is assessed a compensation of $\Delta \pi_{i}^{t+1}$ which is computed as follows

$$
\Delta \pi_{i}^{t+1}=\sum_{k}\left(d_{k}^{t+1}-d_{k}^{t}\right) u_{k}^{t+1}-\sum_{k \neq i}\left(x_{k}^{t+1}-x_{k}^{t}\right) c_{k}^{t+1}-\sum_{k \neq i}\left(\gamma_{k}^{t+1}-\gamma_{k}^{t}\right) \bar{c}_{k}^{t+1}
$$


The total payment and compensation accrued after $t+1$ rounds is given by:

$$
\tau_{i}^{t+1}=\tau_{i}^{t}+\Delta \tau_{i}^{t+1}, \quad \pi_{i}^{t+1}=\pi_{i}^{t}+\Delta \pi_{i}^{t+1}
$$

4. Stopping Rule: The resource manager checks if complementary slackness is approximately verified. A new iteration takes place (step 2) if this condition is not met.

\subsubsection{Analysis}

Let $C_{i}^{E}\left(\gamma_{i}, x_{i}\right):=\mathbb{E}_{\Omega}\left[\bar{C}_{i}\left(\gamma_{i} \Omega, x_{i}\right)\right]$. The computation the partial derivatives of the expected adjustment cost with respect to $\gamma_{i}$ and $x_{i}$ can be an extremely demanding computational task. To account for this fact, we shall relax our definition of truthful participation. In what follows we assume the distribution function of $\Omega$ is common knowledge among demand aggregators and generators. We say that demand aggregators are truthful if $u_{i}^{t+1}=v_{i}^{\prime}\left(d_{i}^{t}\right)$, and that generators are truthful if

$$
\begin{aligned}
c_{i}^{t+1} & =C_{i}^{\prime}\left(x_{i}^{t}\right)+\frac{\partial C_{i}^{E}\left(\gamma_{i}^{t}, x_{i}^{t}\right)}{\partial x_{i}}+\xi_{x, i}^{t} \\
\bar{c}_{i}^{t+1} & =\frac{\partial C_{i}^{E}\left(\gamma_{i}^{t}, x_{i}^{t}\right)}{\partial \gamma_{i}}+\xi_{\gamma, i}^{t}
\end{aligned}
$$

where $\xi_{x, i}^{t}$ and $\xi_{\gamma, i}^{t}$ represent the errors in estimating the partial derivatives of $C_{i}^{E}\left(\gamma_{i}^{t}, x_{i}^{t}\right)$. We assume that they are independent random variables with zero mean and finite variance. Note that a participant may still be truthful even if reporting an incorrect value of marginal expected cost of adjustment. This flexibility comes at a cost in that it is no longer straightforward to design an activity rule (which in this case is tantamount to a statistical test) so as to obtain incentive compatibility in dominant strategies (see Varian [99]). As a result we only obtain incentive compatibility in Nash equilibrium. We now formally state the standing assumptions:

Assumption 7. For each node $i, \mathcal{C}_{i}$ and $\overline{\mathcal{C}}_{i}$ are the sets of strictly convex Lipschitz functions with continuous derivatives, and $\mathcal{V}_{i}$ is the set of strictly concave Lipschitz functions with continuous derivatives. 
Assumption 8. The distribution function of $\Omega$ is common knowledge among demand aggregators and generators.

\section{Convergence}

The strategy for analysis is as follows. First, we will show convergence to optimal dispatch assuming that demand aggregators and generators are truthful. Then we will show that truthful reporting for every participant (demand aggregator or generator) is a Nash equilibrium.

Consider the objective function:

$$
\mathcal{F}(\mathbf{x}, \gamma, \mathbf{d}):=\sum_{i}\left[v_{i}\left(d_{i}\right)-C_{i}\left(x_{i}\right)-C_{i}^{E}\left(\gamma_{i}, x_{i}\right)\right] .
$$

Lemma 4. The sequence $\left\{\mathcal{F}\left(\mathbf{x}^{t}, \gamma^{t}, \mathbf{d}^{t}\right): t>0\right\}$ converges almost surely. Proof. See Appendix C.

Theorem 8. $\left(\mathbf{x}^{t}, \gamma^{t}, \mathbf{d}^{t}\right)$ converges to the unique solution of problem (5.12) with constraints specified in (5.13) almost surely.

Proof. The proof resembles that of Theorem 6 and is omitted here.

\section{Incentive Compatibility}

As the mechanism no longer has an activity rule, it is conceivable that a participant may engage in time-varying reporting strategies not consistent with a concave valuation or convex cost function. This implies that mechanism may fail to close in finite time. This type of behavior seems self-defeating as without market clearing there are no transactions and thus no gains from trade. In what follows we will show that truthful reporting is approximately a Nash equilibrium under the following assumption.

Assumption 9. The net surplus for any demand aggregator or generator is null whenever the mechanism fails to close in finite time.

Theorem 9. Truthful reporting is a $\delta^{M}$-Nash equilibrium, where $\delta^{M}=\frac{1}{2} L D M$. Proof. See Appendix C. 


\section{Participation Constraints and Budget Balance}

Let $\underline{\mathcal{F}}$ denote a lower bound of $\mathcal{F}\left(\mathbf{x}^{*}, \gamma^{*}, \mathbf{d}^{*}\right)$ such that $\underline{\mathcal{F}} \geq \frac{2 I-1}{2 I} \mathcal{F}\left(\mathbf{x}^{*}, \gamma^{*}, \mathbf{d}^{*}\right)$. Let

$$
\tau_{i}^{0}=\underline{\mathcal{F}}, \quad \pi_{i}^{0}=-\underline{\mathcal{F}} .
$$

Assume that $\left(\mathbf{x}^{0}, \gamma^{0}, \mathbf{d}^{0}\right)=(0,0,0)$ and $v_{i}(0)=C_{i}(0)=C_{i}^{E}(0)=0$. Then $\mathcal{F}\left(\mathbf{x}^{0}, \gamma^{0}, \mathbf{d}^{0}\right)=0$. If $\delta^{M} \simeq 0$ it follows from the inequalities obtained in the proof of Theorem 9 that:

$$
\mathbb{E}_{\xi}\left[v_{i}\left(d_{i}^{T}\right)-\tau_{i}^{T}\right] \simeq \mathcal{F}\left(\mathbf{x}^{*}, \gamma^{*}, \mathbf{d}^{*}\right)-\tau_{i}^{0}>0, \quad \mathbb{E}_{\xi}\left[\pi_{i}^{T}-C_{i}\left(x_{i}^{T}\right)\right] \simeq \mathcal{F}\left(\mathbf{x}^{*}, \gamma^{*}, \mathbf{d}^{*}\right)+\pi_{i}^{0}>0
$$

It can be seen that market participants share equally (approximately) the net surplus from trade. This implies that individual rationality constraints are met (assuming an outside option yielding null surplus). To see that the mechanism is approximately budget balanced note that

$$
\begin{aligned}
& \mathbb{E}_{\xi}\left[\tau_{i}^{T}\right] \simeq \tau_{i}^{0}-\left(\sum_{k \neq i} v_{k}\left(d_{k}^{T}\right)-\sum_{k} C_{k}\left(x_{k}^{T}\right)-\sum_{k} C_{k}^{E}\left(\gamma_{k}^{T}, x_{k}^{T}\right)\right), \\
& \mathbb{E}_{\xi}\left[\pi_{i}^{T}\right] \simeq \pi_{i}^{0}+\left(\sum_{k} v_{k}\left(d_{k}^{T}\right)-\sum_{k \neq i} C_{k}\left(x_{k}^{T}\right)-\sum_{k \neq i} C_{k}^{E}\left(\gamma_{k}^{T}, x_{k}^{T}\right)\right)
\end{aligned}
$$

The margin for the market maker is

$$
\sum_{i \in \mathcal{I}}\left(\tau_{i}^{T}-\pi_{i}^{T}\right) \simeq \sum_{i \in \mathcal{I}}\left(\tau_{i}^{0}-\pi_{i}^{0}\right)-(2 I-1) \mathcal{F}\left(\mathbf{x}^{*}, \gamma^{*}, \mathbf{d}^{*}\right) \geq 0
$$

\subsection{Conclusions of this Chapter}

More than thirty years after the first electricity market began operating, the task of properly tuning market rules to ensure economically efficient outcomes (i.e., market design) still seems far from complete. A lot of attention has been paid to direct mechanisms. While direct mechanisms only require one round of interaction, the nature of the information exchange between market participants and the market-maker is quite complex. In such rich strategy space, equilibrium strategic behavior can be quite complex so that it is not easy to find closed-form designs that limit the extent of strategic behavior. 
In this chapter, we have analyzed a different class of mechanisms, namely, iterative mechanisms. In an iterative mechanism, market participants are asked to report the cost or willingness to pay for small adjustments at each iteration and a tentative market dispatch is marginally adjusted ensuring network constraints are satisfied. Small adjustments require less information from market participants at each iteration and this facilitates the identification of incentives for ensuring truthful reporting of private information. At each iteration, each market participant is charged (or compensated) according to the marginal change in surplus to all other market participants (see $[17,37]$ ). We show that truthful reporting is (approximately) a dominant strategy and that when the mechanism stops, the resulting economic dispatch is approximately efficient. Individual participation constraints are satisfied as the net surplus from trade is approximately shared equally by all participants and the mechanism is approximately weakly budget balanced. Interestingly, the mechanism may potentially be suitably altered in various ways as its main properties are not dependent upon the closed-form representation of network constraints but rather on convexity of the set of feasible solutions. Thus, the proposed mechanism may potentially be used in conjunction with convex approximations to AC power flow (see [24,84]). Additionally, to speed up convergence, adjustments may be computed on the basis of an augmented Lagrangian (see Nesterov [70]). We shall analyze these extensions in our future work.

In the second part of this chapter we analyzed an iterative mechanism for stochastic market clearing. The inherent intermittency of renewable technologies poses new challenges for ensuring a reliable and secure operation of electricity markets. In this context, due to estimation error, participants may report incorrect values for the marginal expected cost while still being truthful. We show that truthful reporting is a Nash equilibrium and the resulting dispatch converges almost surely to the efficient dispatch. 


\subsection{Appendix A: Proof of Theorem 6}

\subsubsection{Preliminary Results}

Let

$$
B^{t}=\sum_{i}\left(u_{i}^{t+1} \hat{d}_{i}^{t+1}-c_{i}^{t+1} \hat{x}_{i}^{t+1}\right)-\sum_{i}\left(u_{i}^{t+1} d_{i}^{t}-c_{i}^{t+1} x_{i}^{t}\right)
$$

We want to show now that $B^{t}$ converges to 0 . Noticing that $\sum_{i}\left(u_{i}^{t+1} \hat{d}_{i}^{t+1}-c_{i}^{t+1} \hat{x}_{i}^{t+1}\right)$ is the optimal outcome of problem (5.2) with bids $u_{i}^{t+1}=v_{i}^{\prime}\left(d_{i}^{t}\right)$ and $c_{i}^{t+1}=C_{i}^{\prime}\left(x_{i}^{t}\right)$, let's define

$$
\hat{f}(\mathbf{x}, \mathbf{d}):=\sum_{i}\left(v_{i}^{\prime}\left(d_{i}\right) \hat{d}_{i}-C_{i}^{\prime}\left(x_{i}\right) \hat{x}_{i}\right)
$$

with $\hat{d}_{i}$ and $\hat{x}_{i}$ being the optimal solution to problem (5.2) given inputs $v_{i}^{\prime}\left(d_{i}\right)$ and $C_{i}^{\prime}\left(x_{i}\right)$. Similarly, let

$$
f(\mathbf{x}, \mathbf{d}):=\sum_{i}\left(v_{i}^{\prime}\left(d_{i}\right) d_{i}-C_{i}^{\prime}\left(x_{i}\right) x_{i}\right)
$$

Lemma 5. Function $\hat{f}$ and $f$ are Lipschitz continuous on the feasible region of problem

Proof. Given two arbitrary pairs $\left(\mathbf{x}^{1}, \mathbf{d}^{1}\right)$ and $\left(\mathbf{x}^{2}, \mathbf{d}^{2}\right)$,

$$
\begin{aligned}
\hat{f}\left(\mathbf{x}^{1}, \mathbf{d}^{1}\right) & =\sum_{i}\left(v_{i}^{\prime}\left(d_{i}^{1}\right) \hat{d}_{i}^{1}-C_{i}^{\prime}\left(x_{i}^{1}\right) \hat{x}_{i}^{1}\right) \\
& =\sum_{i}\left[\left(v_{i}^{\prime}\left(d_{i}^{1}\right)-v_{i}^{\prime}\left(d_{i}^{2}\right)+v_{i}^{\prime}\left(d_{i}^{2}\right)\right) \hat{d}_{i}^{1}-\left(C_{i}^{\prime}\left(x_{i}^{1}\right)-C_{i}^{\prime}\left(x_{i}^{2}\right)+C_{i}^{\prime}\left(x_{i}^{2}\right)\right) \hat{x}_{i}^{1}\right] \\
& =\sum_{i}\left[\left(v_{i}^{\prime}\left(d_{i}^{1}\right)-v_{i}^{\prime}\left(d_{i}^{2}\right)\right) \hat{d}_{i}^{1}-\left(C_{i}^{\prime}\left(x_{i}^{1}\right)-C_{i}^{\prime}\left(x_{i}^{2}\right)\right) \hat{x}_{i}^{1}\right]+\sum_{i}\left(v_{i}^{\prime}\left(d_{i}^{2}\right) \hat{d}_{i}^{1}-C_{i}^{\prime}\left(x_{i}^{2}\right) \hat{x}_{i}^{1}\right) \\
& \leq \sum_{i}\left[\left(v_{i}^{\prime}\left(d_{i}^{1}\right)-v_{i}^{\prime}\left(d_{i}^{2}\right)\right) \hat{d}_{i}^{1}-\left(C_{i}^{\prime}\left(x_{i}^{1}\right)-C_{i}^{\prime}\left(x_{i}^{2}\right)\right) \hat{x}_{i}^{1}\right]+\hat{f}\left(\mathbf{x}^{2}, \mathbf{d}^{2}\right) .
\end{aligned}
$$

The inequality is due to the fact that $\hat{f}\left(\mathbf{x}^{2}, \mathbf{d}^{2}\right)$ is the optimal outcome given $v_{i}^{\prime}\left(d_{i}^{2}\right)$ and $C_{i}^{\prime}\left(x_{i}^{2}\right)$. Similarly,

$$
\hat{f}\left(\mathbf{x}^{2}, \mathbf{d}^{2}\right) \leq \sum_{i}\left[\left(v_{i}^{\prime}\left(d_{i}^{2}\right)-v_{i}^{\prime}\left(d_{i}^{1}\right)\right) \hat{d}_{i}^{2}-\left(C_{i}^{\prime}\left(x_{i}^{2}\right)-C_{i}^{\prime}\left(x_{i}^{1}\right)\right) \hat{x}_{i}^{2}\right]+\hat{f}\left(\mathbf{d}^{1}, \mathbf{x}^{1}\right)
$$


Since $v_{i}^{\prime}(\cdot)$ and $C_{i}^{\prime}(\cdot)$ are Lipschitz continuous by Assumption 6, Lipschitz continuity of $\hat{f}$ follows. Analogously, we can show that $f$ is Lipschitz continuous too.

The convergence result of $B^{t}=\hat{f}\left(\mathbf{x}^{t}, \mathbf{d}^{t}\right)-f\left(\mathbf{x}^{t}, \mathbf{d}^{t}\right)$ is given by the following lemma.

Lemma 6. The sequence $\left\{B^{t}: t>0\right\}$ converges to 0 .

Proof. In light of (5.7) and (5.20),

$$
\mathcal{F}\left(\mathbf{x}^{t+1}, \mathbf{d}^{t+1}\right) \geq \mathcal{F}\left(\mathbf{x}^{t}, \mathbf{d}^{t}\right)+\alpha_{t} B^{t}-\frac{\alpha_{t}^{2}}{2} L D .
$$

It follows that

$$
\lim _{t} \mathcal{F}\left(\mathbf{x}^{t}, \mathbf{d}^{t}\right) \geq \sum_{t} \alpha_{t} B^{t}-\frac{1}{2} \sum_{t} \alpha_{t}^{2} L D .
$$

Since $B^{t} \geq 0, \sum_{t} \alpha_{t}^{2}<\infty$ and the sequence $\left\{\mathcal{F}\left(\mathbf{x}^{t}, \mathbf{d}^{t}\right): t>0\right\}$ converges by Lemma 3 ,

$$
\sum_{t} \alpha_{t} B^{t}<\infty
$$

By Lemma $5, \hat{f}$ and $f$ are Lipschitz continuous. Hence,

$$
\begin{aligned}
B^{t+1}-B^{t} & =\left[\hat{f}\left(\mathbf{x}^{t+1}, \mathbf{d}^{t+1}\right)-f\left(\mathbf{x}^{t+1}, \mathbf{d}^{t+1}\right)\right]-\left[\hat{f}\left(\mathbf{x}^{t}, \mathbf{d}^{t}\right)-f\left(\mathbf{x}^{t}, \mathbf{d}^{t}\right)\right] \\
& \leq L_{B}\left(\left|\mathbf{d}^{t+1}-\mathbf{d}^{t}\right|+\left|\mathbf{x}^{t+1}-\mathbf{x}^{t}\right|\right) \\
& =\alpha_{t} L_{B}\left(\left|\hat{\mathbf{d}}^{t+1}-\mathbf{d}^{t}\right|+\left|\hat{\mathbf{x}}^{t+1}-\mathbf{x}^{t}\right|\right) \\
& \leq \alpha_{t} K
\end{aligned}
$$

for some $L_{B}, K>0$.

Assume by contradiction that $B^{t}$ does not converge to 0 . Then there exists $\epsilon>0$ such that $B^{t}>\epsilon$ infinitely often. Since $\sum_{t=1}^{\infty} \alpha_{t} B^{t}$ is finite, $B^{t}<\epsilon / 2$ for infinite $t$ 's too. We select a subsequence $\left\{B^{t(k)}: k>0\right\}$ of $\left\{B^{t}: t>0\right\}$ as follows.

(i) $t(1)=1$. 
(ii) $\forall m>0$,

$$
\begin{aligned}
\tilde{t}(2 m) & =\inf \left\{t>t(2 m-1): B^{t}<\epsilon / 2\right\}, \\
t(2 m+1) & =\inf \left\{t>\tilde{t}(2 m): B^{t}>\epsilon\right\}, \\
t(2 m) & =\sup \left\{t<t(2 m+1): B^{t}<\epsilon / 2 \leq B^{t+1}\right\} .
\end{aligned}
$$

It is implied that $B^{t(2 m)}<\epsilon / 2, B^{t(2 m+1)}>\epsilon$, and for all $t$ with $t(2 m)<t<t(2 m+1)$, $2 / \epsilon \leq B^{t} \leq \epsilon$. Now in light of (5.22),

$$
\epsilon / 2<B^{t(2 m+1)}-B^{t(2 m)} \leq \sum_{t=t(2 m)+1}^{t(2 m+1)} \alpha_{t} K .
$$

It follows that

$$
\sum_{t=t(2 m)+1}^{t(2 m+1)} \alpha_{t}>\frac{\epsilon}{2 K}
$$

Therefore,

$$
\sum_{t=t(2 m)+1}^{t(2 m+1)} \alpha_{t} B^{t} \geq \epsilon / 2 \sum_{t=t(2 m)+1}^{t(2 m+1)} \alpha_{t}>\frac{\epsilon^{2}}{4 K}
$$

and

$$
\sum_{t} \alpha_{t} B^{t} \geq \sum_{m} \sum_{t=t(2 m)+1}^{t(2 m+1)} \alpha_{t} B^{t}=\infty .
$$

This contradicts (5.21).

We are now in a position to show the convergence of $\left(\mathbf{x}^{t}, \mathbf{d}^{t}\right)$.

\subsubsection{Proof of Theorem 6}

By Weierstrass theorem, $\left(\mathbf{x}^{t}, \mathbf{d}^{t}\right)$ has a converging subsequence $\left(\mathbf{x}^{t(k)}, \mathbf{d}^{t(k)}\right), k=1,2, \ldots$ Denote its limit by $\left(\mathbf{x}^{*}, \mathbf{d}^{*}\right)$. Then

$$
B^{t(k)}=\hat{f}\left(\mathbf{x}^{t(k)}, \mathbf{d}^{t(k)}\right)-f\left(\mathbf{x}^{t(k)}, \mathbf{d}^{t(k)}\right)
$$


converges to 0 as a subsequence of $B^{t}$. Since $\hat{f}$ and $f$ are continuous,

$$
\lim _{k} B^{t(k)}=\lim _{k} \hat{f}\left(\mathbf{x}^{t(k)}, \mathbf{d}^{t(k)}\right)-\lim _{k} f\left(\mathbf{x}^{t(k)}, \mathbf{d}^{t(k)}\right)=\hat{f}\left(\mathbf{x}^{*}, \mathbf{d}^{*}\right)-f\left(\mathbf{x}^{*}, \mathbf{d}^{*}\right)=0 .
$$

We now have $\hat{f}\left(\mathbf{x}^{*}, \mathbf{d}^{*}\right)=f\left(\mathbf{x}^{*}, \mathbf{d}^{*}\right)$, i.e., $\left(\mathbf{x}^{*}, \mathbf{d}^{*}\right)$ is a solution to problem (5.2) with inputs $v_{i}^{\prime}\left(d_{i}^{*}\right)$ and $C_{i}^{\prime}\left(x_{i}^{*}\right)$. In other words,

$$
\sum_{i}\left(v_{i}^{\prime}\left(d_{i}^{*}\right)\left(d_{i}^{*}-d_{i}\right)-C_{i}^{\prime}\left(x_{i}^{*}\right)\left(x_{i}^{*}-x_{i}\right)\right) \geq 0
$$

for all $x_{i}$ and $d_{i}$ satisfying the constraints in (5.1). This is sufficient for $\left(\mathbf{x}^{*}, \mathbf{d}^{*}\right)$ to be a solution of the original problem (5.1) (see Nesterov [70] Theorem 2.2.5).

Assuming by contradiction that $\left(\mathbf{x}^{t}, \mathbf{d}^{t}\right)$ does not converge, then it has at least two subsequences converging to two different limiting points, both of which are optimal. Since the original problem admits a unique solution, this is a contradiction. Therefore, $\left(\mathbf{x}^{t}, \mathbf{d}^{t}\right)$ converges and its limiting point $\left(\mathbf{x}^{*}, \mathbf{d}^{*}\right)$ is exactly the unique solution to problem (5.1).

\subsection{Appendix B: Demand Aggregator and Generator Associated with Multiple Units}

In this section, we show that the previous results can be generalized to the case that each demand aggregator/ generator is associated to multiple units/nodes. For ease of exposition, we consider a one-stage market model. Let $\mathcal{A}$ and $\mathcal{G}$ denote the set of demand aggregators and generators, respectively. The optimal economic dispatch constrained by the power balance and line power flow limits in this case is the solution to:

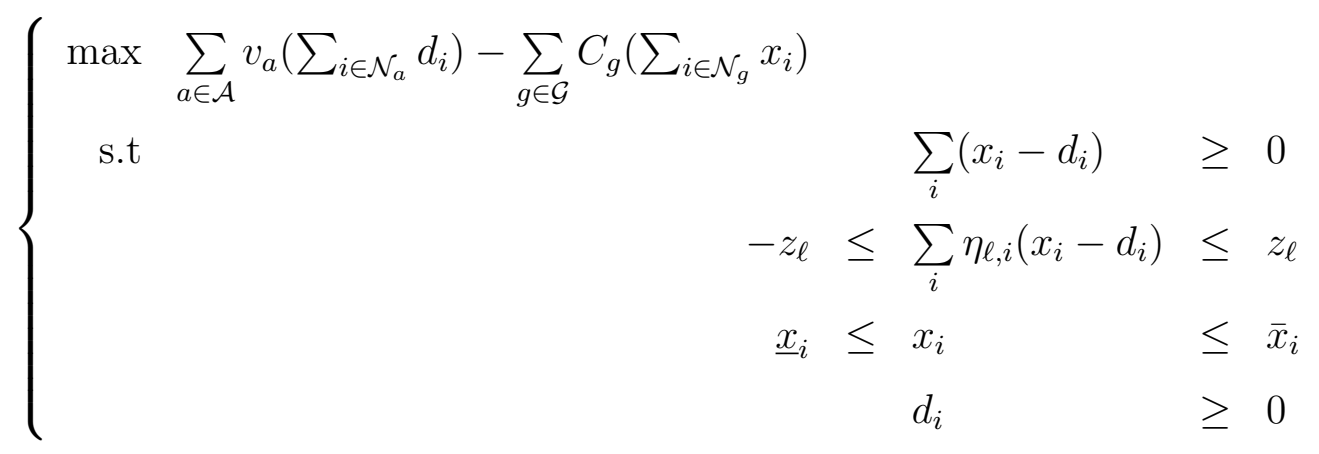


Here $\mathcal{N}_{a}$ and $\mathcal{N}_{g}$ denote the set of nodes associated with demand aggregator $a$ and generator $g$, respectively.

\subsubsection{Mechanism Design}

The mechanism works as follows.

1. Initialization. Start with any feasible pair $\left(\mathbf{x}^{0}, \mathbf{d}^{0}\right)$.

2. Schedule and Price updates. At each round (or iteration) $t \geq 0$ :

(a) Price update: Each demand aggregator and each generator respectively submit bids $u_{a}^{t+1}$ and $c_{g}^{t+1}$. The market maker solves

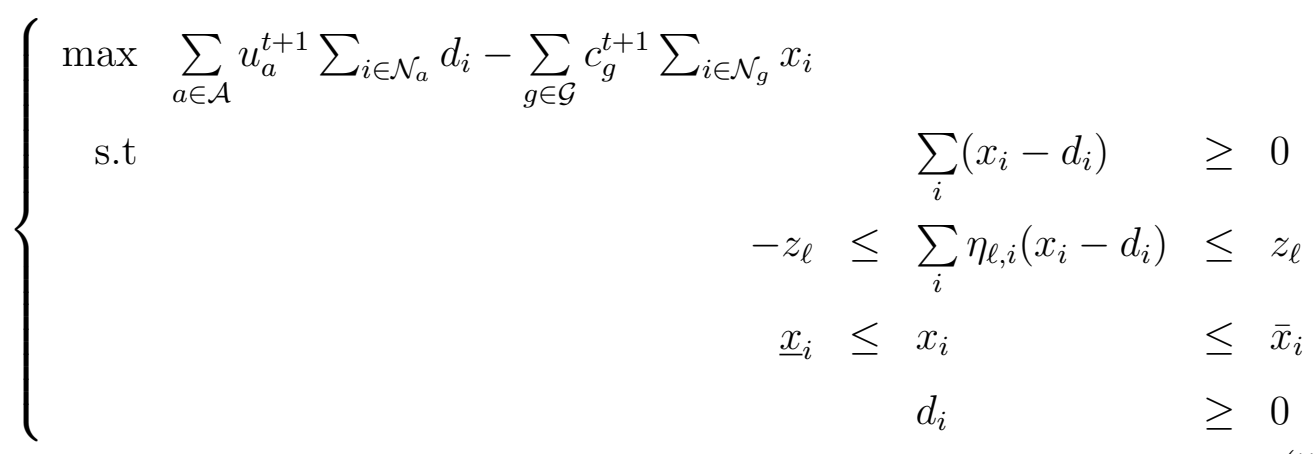

(b) Demand and Generation updates: Let $\left(\hat{\mathbf{x}}^{t+1}, \hat{\mathbf{d}}^{t+1}\right)$ denote the optimal solution to (5.24). The tentative schedules for production and consumption are updated as follows:

$$
\mathbf{x}^{t+1}=\mathbf{x}^{t}+\alpha_{t}\left(\hat{\mathbf{x}}^{t+1}-\mathbf{x}^{t}\right)
$$

and

$$
\mathbf{d}^{t+1}=\mathbf{d}^{t}+\alpha_{t}\left(\hat{\mathbf{d}}^{t+1}-\mathbf{d}^{t}\right)
$$

where $\left\{\alpha_{t}: t \geq 0\right\}$ is a sequence of stepsizes that satisfy $\alpha_{t} \in(0,1), \alpha_{t} \rightarrow 0^{+}$and

$$
\sum_{t=1}^{\infty} \alpha_{t}=\infty, \quad \sum_{t=0}^{\infty} \alpha_{t}^{2}=M<\infty
$$


3. Incentive Payment: Each demand aggregator $a \in \mathcal{A}$ is assessed a payment of $\tau_{a}^{t+1}$ which is computed as follows

$$
\Delta \tau_{a}^{t+1}=-\sum_{k \neq a, k \in \mathcal{A}} u_{k}^{t+1} \sum_{i \in \mathcal{N}_{k}}\left(d_{i}^{t+1}-d_{i}^{t}\right)+\sum_{h \in \mathcal{G}} c_{h}^{t+1} \sum_{i \in \mathcal{N}_{h}}\left(x_{i}^{t+1}-x_{i}^{t}\right) .
$$

Each generator $g \in \mathcal{G}$ is assessed a payment of $\tau_{g}^{t+1}$ which is computed as follows

$$
\Delta \tau_{g}^{t+1}=\sum_{k \in \mathcal{A}} u_{k}^{t+1} \sum_{i \in \mathcal{N}_{k}}\left(d_{i}^{t+1}-d_{i}^{t}\right)-\sum_{h \neq g, h \in \mathcal{G}} c_{h}^{t+1} \sum_{i \in \mathcal{N}_{h}}\left(x_{i}^{t+1}-x_{i}^{t}\right)
$$

The total payment and compensation accrued after $t+1$ rounds is given by:

$$
\tau_{a}^{t+1}=\tau_{a}^{t}+\Delta \tau_{a}^{t+1}, \quad \pi_{g}^{t+1}=\pi_{g}^{t}+\Delta \pi_{g}^{t+1}
$$

4. Activity Rule: The market-maker checks that

$$
\begin{aligned}
& {\left[\left(\sum_{i \in \mathcal{N}_{a}} d_{i}^{\tilde{t}}\right)-\left(\sum_{i \in \mathcal{N}_{a}} d_{i}^{t}\right)\right] \cdot\left(u_{a}^{\tilde{t}+1}-u_{a}^{t+1}\right)<0,} \\
& {\left[\left(\sum_{i \in \mathcal{N}_{g}} x_{i}^{\tilde{t}}\right)-\left(\sum_{i \in \mathcal{N}_{g}} x_{i}^{t}\right)\right] \cdot\left(c_{i}^{\tilde{t}+1}-c_{g}^{t+1}\right)>0}
\end{aligned}
$$

for all $\tilde{t}<t$ and for each generator and demand aggregator located in node $i$. A participant is removed from market-clearing when this activity rule is violated.

5. Stopping Rule: The resource manager checks if complementary slackness is approximately verified. A new iteration takes place (step 2) if this condition is not met.

\section{Truthful Participation}

We say that demand aggregators are truthful if

$$
u_{a}^{t+1}=v_{a}^{\prime}\left(\sum_{i \in \mathcal{N}_{a}} d_{i}^{t}\right) .
$$

Also, generators are truthful if

$$
c_{g}^{t+1}=C_{g}^{\prime}\left(\sum_{i \in \mathcal{N}_{g}} x_{i}^{t}\right)
$$




\subsubsection{Analysis}

The strategy for analysis resembles that in Section 5.4. First, assuming that demand aggregators and generators are truthful we will show that convergence to optimal dispatch. Then we will show that it is optimal for every participant (demand aggregator or generator) to be truthful.

\section{Convergence}

Define

$$
\begin{aligned}
D_{a} & :=\sum_{i \in \mathcal{N}_{a}} d_{i} \\
X_{g} & :=\sum_{i \in \mathcal{N}_{g}} x_{i} .
\end{aligned}
$$

Assumption 10. In the set of solutions to problem (5.23), $\left\{D_{a}: a \in \mathcal{A}\right\}$ and $\left\{X_{g}: g \in \mathcal{G}\right\}$ are unique.

In the following analysis we assume that Assumption 6 and 10 hold. Consider the objective function

$$
\mathcal{F}^{m}(\mathbf{x}, \mathbf{d}):=\sum_{a \in \mathcal{A}} v_{a}\left(\sum_{i \in \mathcal{N}_{a}} d_{i}\right)-\sum_{g \in \mathcal{G}} C_{g}\left(\sum_{i \in \mathcal{N}_{g}} x_{i}\right) .
$$

Lemma 7. $\left\{\mathcal{F}^{m}\left(\mathbf{x}^{t}, \mathbf{d}^{t}\right): t>0\right\}$ converges.

Proof. Since $\mathcal{F}^{m}(\mathbf{x}, \mathbf{d})$ has a Lipschitz continuous gradient,

$$
\begin{aligned}
\mathcal{F}^{m}\left(\mathbf{x}^{t+1}, \mathbf{d}^{t+1}\right) \geq & \mathcal{F}^{m}\left(\mathbf{x}^{t}, \mathbf{d}^{t}\right)+\alpha_{t} \nabla_{\mathbf{d}} \mathcal{F}^{m}\left(\mathbf{x}^{t}, \mathbf{d}^{t}\right) \cdot\left(\hat{\mathbf{d}}^{t+1}-\mathbf{d}^{t}\right)+\alpha_{t} \nabla_{\mathbf{x}} \mathcal{F}^{m}\left(\mathbf{x}^{t}, \mathbf{d}^{t}\right) \cdot\left(\hat{\mathbf{x}}^{t+1}-\mathbf{x}^{t}\right) \\
& -\frac{\alpha_{t}^{2}}{2} L D \\
= & \mathcal{F}^{m}\left(\mathbf{x}^{t}, \mathbf{d}^{t}\right)+\alpha_{t} \sum_{a \in \mathcal{A}} \sum_{i \in \mathcal{N}_{a}}\left[\left(\hat{d}_{i}^{t+1}-d_{i}^{t}\right) \cdot v_{a}^{\prime}\left(\sum_{j \in \mathcal{N}_{a}} d_{j}^{t}\right)\right] \\
& -\alpha_{t} \sum_{g \in \mathcal{G}} \sum_{i \in \mathcal{N}_{g}}\left[\left(\hat{x}_{i}^{t+1}-x_{i}^{t}\right) \cdot C_{g}^{\prime}\left(\sum_{j \in \mathcal{N}_{g}} x_{j}^{t}\right)\right]-\frac{\alpha_{t}^{2}}{2} L D \\
= & \mathcal{F}^{m}\left(\mathbf{x}^{t}, \mathbf{d}^{t}\right)+\alpha_{t}\left(\sum_{a \in \mathcal{A}} u_{a}^{t+1} \sum_{i \in \mathcal{N}_{a}} \hat{d}_{i}^{t+1}-\sum_{g \in \mathcal{G}} c_{g}^{t+1} \sum_{i \in \mathcal{N}_{g}} \hat{x}_{i}^{t+1}\right)
\end{aligned}
$$




$$
\begin{aligned}
& -\alpha_{t}\left(\sum_{a \in \mathcal{A}} u_{a}^{t+1} \sum_{i \in \mathcal{N}_{a}} d_{i}^{t}-\sum_{g \in \mathcal{G}} c_{g}^{t+1} \sum_{i \in \mathcal{N}_{g}} x_{i}^{t}\right)-\frac{\alpha_{t}^{2}}{2} L D \\
\geq & \mathcal{F}^{m}\left(\mathbf{x}^{t}, \mathbf{d}^{t}\right)-\frac{\alpha_{t}^{2}}{2} L D,
\end{aligned}
$$

where the last inequality is due to the fact that $\left(\hat{\mathbf{x}}^{t+1}, \hat{\mathbf{d}}^{t+1}\right)$ is optimal solution to (5.24) for which $\left(\mathbf{x}^{t}, \mathbf{d}^{t}\right)$ is a feasible solution. The convergence of $\left\{\mathcal{F}^{m}\left(\mathbf{x}^{t}, \mathbf{d}^{t}\right): t>0\right\}$ now follows.

Theorem 10. Assuming that generators and demand aggregators are truthful, $\left(\mathbf{X}^{t}, \mathbf{D}^{t}\right)$ converges to the unique solution of problem (5.23).

Proof. The proof resembles that of Theorem 6 and is omitted here.

\section{Incentive Compatibility}

We first show that truthful reporting is approximately a dominant strategy.

Theorem 11. Truthful reporting is a $\delta^{M}>0$ dominant strategy where $\delta^{M}=\frac{1}{2} L D M$.

Proof. We start by showing that truthful reporting is a $\delta^{M}$-Nash equilibrium. Recall that

$$
\begin{aligned}
& u_{a}^{t+1}=v_{a}^{\prime}\left(\sum_{i \in \mathcal{N}_{a}} d_{i}^{t}\right)=v_{a}^{\prime}\left(D_{a}^{t}\right), \\
& c_{g}^{t+1}=C_{g}^{\prime}\left(\sum_{i \in \mathcal{N}_{g}} x_{i}^{t}\right)=C_{g}^{\prime}\left(X_{g}^{t}\right) .
\end{aligned}
$$

After $T$ rounds the total payment for demand aggregator $a$ is

$$
\begin{aligned}
\tau_{a}^{T} & =\tau_{a}^{0}-\sum_{t=0}^{T-1} \sum_{k \neq a, k \in \mathcal{A}} u_{k}^{t+1} \sum_{i \in \mathcal{N}_{k}}\left(d_{i}^{t+1}-d_{i}^{t}\right)+\sum_{t=0}^{T-1} \sum_{h \in \mathcal{G}} c_{h}^{t+1} \sum_{i \in \mathcal{N}_{h}}\left(x_{i}^{t+1}-x_{i}^{t}\right) \\
& =\tau_{a}^{0}-\sum_{t=0}^{T-1} \sum_{k \neq a, k \in \mathcal{A}} v_{k}^{\prime}\left(D_{k}^{t}\right)\left(D_{k}^{t+1}-D_{k}^{t}\right)+\sum_{t=0}^{T-1} \sum_{h \in \mathcal{G}} C_{h}^{\prime}\left(X_{h}^{t}\right)\left(X_{h}^{t+1}-X_{h}^{t}\right)
\end{aligned}
$$

and the total compensation for generator $g$ is

$$
\begin{aligned}
\pi_{g}^{T} & =\pi_{g}^{0}+\sum_{t=0}^{T-1} \sum_{k \in \mathcal{A}} u_{k}^{t+1} \sum_{i \in \mathcal{N}_{k}}\left(d_{i}^{t+1}-d_{i}^{t}\right)-\sum_{t=0}^{T-1} \sum_{h \neq g, h \in \mathcal{G}} c_{h}^{t+1} \sum_{i \in \mathcal{N}_{h}}\left(x_{i}^{t+1}-x_{i}^{t}\right) \\
& =\pi_{g}^{0}+\sum_{t=0}^{T-1} \sum_{k \in \mathcal{A}} v_{k}^{\prime}\left(D_{k}^{t}\right)\left(D_{k}^{t+1}-D_{k}^{t}\right)-\sum_{t=0}^{T-1} \sum_{h \neq g, h \in \mathcal{G}} C_{h}^{\prime}\left(X_{h}^{t}\right)\left(X_{h}^{t+1}-X_{h}^{t}\right)
\end{aligned}
$$


In light of Assumption 6,

$$
\tau_{a}^{T} \geq \tau_{a}^{0}-\left(\sum_{k \neq a, k \in \mathcal{A}} v_{k}\left(D_{k}^{T}\right)-\sum_{h \in \mathcal{G}} C_{h}\left(X_{h}^{T}\right)\right)+\left(\sum_{k \neq a, k \in \mathcal{A}} v_{k}\left(D_{k}^{0}\right)-\sum_{h \in \mathcal{G}} C_{h}\left(X_{h}^{0}\right)\right),
$$

and

$$
\tau_{a}^{T} \leq \tau_{a}^{0}-\left(\sum_{k \neq a, k \in \mathcal{A}} v_{k}\left(D_{k}^{T}\right)-\sum_{h \in \mathcal{G}} C_{h}\left(X_{h}^{T}\right)\right)+\left(\sum_{k \neq a, k \in \mathcal{A}} v_{k}\left(D_{k}^{0}\right)-\sum_{h \in \mathcal{G}} C_{h}\left(X_{h}^{0}\right)\right)-\frac{1}{2} L D M .
$$

Analogously,

$$
\pi_{g}^{T} \geq \pi_{g}^{0}+\sum_{k \in \mathcal{A}} v_{k}\left(D_{k}^{T}\right)-\sum_{h \neq g, h \in \mathcal{G}} C_{h}\left(X_{h}^{T}\right)-\sum_{k \in \mathcal{A}} v_{k}\left(D_{k}^{0}\right)+\sum_{h \neq g, h \in \mathcal{G}} C_{h}\left(X_{h}^{0}\right)
$$

and

$$
\pi_{g}^{T} \leq \pi_{g}^{0}+\sum_{k \in \mathcal{A}} v_{k}\left(D_{k}^{T}\right)-\sum_{h \neq g, h \in \mathcal{G}} C_{h}\left(X_{h}^{T}\right)-\sum_{k \in \mathcal{A}} v_{k}\left(D_{k}^{0}\right)+\sum_{h \neq g, h \in \mathcal{G}} C_{h}\left(X_{h}^{0}\right)+\frac{1}{2} L D M .
$$

It follows that the net surplus for demand aggregator $a$ satisfies

$$
\begin{aligned}
v_{a}\left(D_{a}^{T}\right)-\tau_{a}^{T} \in\left[\mathcal{F}^{m}\left(\mathbf{x}^{T}, \mathbf{d}^{T}\right)-\mathcal{F}^{m}\right. & \left(\mathbf{x}^{0}, \mathbf{d}^{0}\right)+v_{a}\left(D_{a}^{0}\right)-\tau_{a}^{0}, \\
& \left.\mathcal{F}^{m}\left(\mathbf{x}^{T}, \mathbf{d}^{T}\right)-\mathcal{F}^{m}\left(\mathbf{x}^{0}, \mathbf{d}^{0}\right)+v_{a}\left(D_{a}^{0}\right)-\tau_{a}^{0}+\frac{1}{2} L D M\right] .
\end{aligned}
$$

As $T \rightarrow \infty$,

$$
v_{a}\left(D_{a}^{T}\right)-\tau_{a}^{T} \geq \mathcal{F}^{m}\left(\mathbf{x}^{*}, \mathbf{d}^{*}\right)-\mathcal{F}^{m}\left(\mathbf{x}^{0}, \mathbf{d}^{0}\right)+v_{a}\left(D_{a}^{0}\right)-\tau_{a}^{0}
$$

Consistently untruthful behavior leads to a suboptimal solution say $\left(\tilde{\mathbf{x}}^{*}, \tilde{\mathbf{d}}^{*}\right)$. Then,

$$
\begin{aligned}
v_{a}\left(D_{a}^{T}\right)-\tau_{a}^{T} & \geq \mathcal{F}^{m}\left(\mathbf{x}^{*}, \mathbf{d}^{*}\right)-\mathcal{F}^{m}\left(\mathbf{x}^{0}, \mathbf{d}^{0}\right)+v_{a}\left(D_{a}^{0}\right)-\tau_{a}^{0} \\
& \geq \mathcal{F}^{m}\left(\tilde{\mathbf{x}}^{*}, \tilde{\mathbf{d}}^{*}\right)-\mathcal{F}^{m}\left(\mathbf{x}^{0}, \mathbf{d}^{0}\right)+v_{a}\left(D_{a}^{0}\right)-\tau_{a}^{0} \\
& \geq v_{a}\left(\tilde{D}_{a}^{T}\right)-\tilde{\tau}_{a}^{T}-\frac{1}{2} L D M
\end{aligned}
$$

It is $\delta^{M}$-optimal for demand aggregator $i$ to be truthful when all other market participants are truthful. 
The net surplus for generator $g$ satisfies

$$
\begin{aligned}
\pi_{g}^{T}-C_{g}\left(X_{g}^{T}\right) \in\left[\mathcal{F}^{m}\left(\mathbf{x}^{T}, \mathbf{d}^{T}\right)-\mathcal{F}^{m}\left(\mathbf{x}^{0}, \mathbf{d}^{0}\right)-C_{g}\left(X_{g}^{0}\right)+\pi_{g}^{0},\right. \\
\left.\mathcal{F}^{m}\left(\mathbf{x}^{T}, \mathbf{d}^{T}\right)-\mathcal{F}^{m}\left(\mathbf{x}^{0}, \mathbf{d}^{0}\right)-C_{g}\left(X_{g}^{0}\right)+\pi_{g}^{0}+\frac{1}{2} L D M\right] .
\end{aligned}
$$

In the limit as $T \rightarrow \infty$,

$$
\begin{aligned}
\pi_{g}^{T}-C_{g}\left(X_{g}^{T}\right) & \geq \mathcal{F}^{m}\left(\mathbf{x}^{*}, \mathbf{d}^{*}\right)-\mathcal{F}^{m}\left(\mathbf{x}^{0}, \mathbf{d}^{0}\right)-C_{g}\left(X_{g}^{0}\right)+\pi_{g}^{0} \\
& \geq \mathcal{F}^{m}\left(\tilde{\mathbf{x}}^{T}, \tilde{\mathbf{d}}^{T}\right)-\mathcal{F}^{m}\left(\mathbf{x}^{0}, \mathbf{d}^{0}\right)-C_{g}\left(X_{g}^{0}\right)+\pi_{g}^{0} \\
& \geq \tilde{\pi}_{g}^{T}-C_{g}\left(\tilde{X}_{g}^{T}\right)-\frac{1}{2} L D M .
\end{aligned}
$$

Therefore, It is also $\delta^{M}$-optimal for generator $g$ to be truthful when all other market participants are truthful.

Similar to the analysis in Section 5.4.2, we can generalize the previous result to show that truthful reporting is a $\delta^{M}$-dominant strategy for all market participants.

\section{Participation Constraints and Budget Balance}

Let $\underline{\mathcal{F}}^{m}$ denote a lower bound of $\mathcal{F}^{m}\left(\mathbf{x}^{*}, \mathbf{d}^{*}\right)$ such that $\underline{\mathcal{F}}^{m} \geq \frac{|\mathcal{A}|+|\mathcal{G}|-1}{|\mathcal{A}|+|\mathcal{G}|} \mathcal{F}^{m}\left(\mathbf{x}^{*}, \mathbf{d}^{*}\right)$. Let

$$
\tau_{a}^{0}=\underline{\mathcal{F}}, \quad \pi_{g}^{0}=-\underline{\mathcal{F}} .
$$

Assume that $\left(\mathbf{x}^{0}, \mathbf{d}^{0}\right)=(0,0)$ and $v_{i}(0)=C_{i}(0)=0$ so that $\mathcal{F}^{m}\left(\mathbf{x}^{0}, \mathbf{d}^{0}\right)=0$. If $\delta^{M} \simeq 0$ it follows from the inequalities obtained in the proof of Theorem (11) that:

$$
v_{a}\left(D_{a}^{T}\right)-\tau_{a}^{T} \simeq \mathcal{F}^{m}\left(\mathbf{x}^{*}, \mathbf{d}^{*}\right)-\tau_{a}^{0}>0, \quad \pi_{g}^{T}-C_{g}\left(x_{g}^{T}\right) \simeq \mathcal{F}^{m}\left(\mathbf{x}^{*}, \mathbf{d}^{*}\right)+\pi_{g}^{0}>0 .
$$

It can be seen that market participants share equally (approximately) the net surplus from trade. This implies that individual rationality constraints are met (assuming an outside option yielding null surplus). To see that the mechanism is approximately budget balanced note that

$$
\begin{aligned}
\tau_{a}^{T} & \simeq \tau_{a}^{0}-\left(\sum_{k \neq a, k \in \mathcal{A}} v_{k}\left(D_{k}^{T}\right)-\sum_{h \in \mathcal{G}} C_{h}\left(X_{h}^{T}\right)\right), \\
\pi_{g}^{T} & \simeq \pi_{g}^{0}+\sum_{k \in \mathcal{A}} v_{k}\left(D_{k}^{T}\right)-\sum_{h \neq g, h \in \mathcal{G}} C_{h}\left(X_{h}^{T}\right) .
\end{aligned}
$$


The margin for the market maker is

$$
\sum_{a \in \mathcal{A}} \tau_{a}^{T}-\sum_{g \in \mathcal{G}} \pi_{g}^{T} \simeq \sum_{a \in \mathcal{A}} \tau_{a}^{0}-\sum_{g \in \mathcal{G}} \pi_{g}^{0}-(|\mathcal{A}|+|\mathcal{G}|-1) \mathcal{F}^{m}\left(\mathbf{x}^{*}, \mathbf{d}^{*}\right) \geq 0
$$

\subsection{Appendix C: Proofs for Stochastic Market Clear- ing}

\subsubsection{Proof of Lemma 4}

By Assumption $6, v_{i}^{\prime}(\cdot), C_{i}^{\prime}(\cdot)$ and $\nabla \bar{C}_{i}(\cdot, \cdot)$ are Lipschitz continuous for all $i \in \mathcal{I}$. Hence $\mathcal{F}(\mathbf{x}, \gamma, \mathbf{d})$ has Lipschitz continuous gradient so that

$$
\begin{array}{r}
\mathcal{F}\left(\mathbf{x}^{t+1}, \gamma^{t+1}, \mathbf{d}^{t+1}\right) \geq \mathcal{F}\left(\mathbf{x}^{t}, \gamma^{t}, \mathbf{d}^{t}\right)+\nabla_{\mathbf{d}} \mathcal{F}\left(\mathbf{x}^{t}, \gamma^{t}, \mathbf{d}^{t}\right) \cdot\left(\mathbf{d}^{t+1}-\mathbf{d}^{t}\right)+\nabla_{\mathbf{x}} \mathcal{F}\left(\mathbf{x}^{t}, \gamma^{t}, \mathbf{d}^{t}\right) \cdot\left(\mathbf{x}^{t+1}-\mathbf{x}^{t}\right) \\
+\nabla_{\gamma} \mathcal{F}\left(\mathbf{x}^{t}, \gamma^{t}, \mathbf{d}^{t}\right) \cdot\left(\gamma^{t+1}-\gamma^{t}\right)-\frac{1}{2} L\left\|\mathbf{d}^{t+1}+\gamma^{t+1}+\mathbf{x}^{t+1}-\mathbf{d}^{t}-\gamma^{t}-\mathbf{x}^{t}\right\|^{2}
\end{array}
$$

for some Lipschitz constant $L>0$. Given that $\mathbf{x}^{t+1}=\mathbf{x}^{t}+\alpha_{t}\left(\hat{\mathbf{x}}^{t+1}-\mathbf{x}^{t}\right), \gamma^{t+1}=\gamma^{t}+$ $\alpha_{t}\left(\hat{\gamma}^{t+1}-\gamma^{t}\right)$ and $\mathbf{d}^{t+1}=\mathbf{d}^{t}+\alpha_{t}\left(\hat{\mathbf{d}}^{t+1}-\mathbf{d}^{t}\right)$, the inequality can be rewritten as

$$
\begin{aligned}
\mathcal{F}\left(\mathbf{x}^{t+1}, \gamma^{t+1}, \mathbf{d}^{t+1}\right) \geq & \mathcal{F}\left(\mathbf{x}^{t}, \gamma^{t}, \mathbf{d}^{t}\right)+\alpha_{t} \nabla_{\mathbf{d}} \mathcal{F}\left(\mathbf{x}^{t}, \gamma^{t}, \mathbf{d}^{t}\right) \cdot\left(\hat{\mathbf{d}}^{t+1}-\mathbf{d}^{t}\right) \\
& +\alpha_{t} \nabla_{\mathbf{x}} \mathcal{F}\left(\mathbf{x}^{t}, \gamma^{t}, \mathbf{d}^{t}\right) \cdot\left(\hat{\mathbf{x}}^{t+1}-\mathbf{x}^{t}\right)+\alpha_{t} \nabla_{\gamma} \mathcal{F}\left(\mathbf{x}^{t}, \gamma^{t}, \mathbf{d}^{t}\right) \cdot\left(\hat{\gamma}^{t+1}-\gamma^{t}\right) \\
& -\frac{\alpha_{t}^{2}}{2} L\left\|\hat{\mathbf{d}}^{t+1}+\hat{\mathbf{x}}^{t+1}+\hat{\gamma}^{t+1}-\mathbf{d}^{t}-\mathbf{x}^{t}-\gamma^{t}\right\|^{2} .
\end{aligned}
$$

The constraints in (5.13) give a compact feasible region, implying that $\hat{\mathbf{d}}^{t+1}$ and $\hat{\mathbf{x}}^{t+1}$ are bounded. Noticing that $\mathbf{d}^{t+1}=\left(1-\alpha_{t}\right) \mathbf{d}^{t}+\alpha_{t} \hat{\mathbf{d}}^{t+1}, \mathbf{x}^{t+1}=\left(1-\alpha_{t}\right) \mathbf{x}^{t}+\alpha_{t} \hat{\mathbf{x}}^{t+1}$ and $\gamma^{t+1}=\left(1-\alpha_{t}\right) \gamma^{t}+\alpha_{t} \hat{\gamma}^{t+1}$, a simple induction indicates that $\mathbf{d}^{t+1}, \mathbf{x}^{t+1}$ and $\gamma^{t+1}$ are also bounded. Therefore, $\left\|\hat{\mathbf{d}}^{t+1}+\hat{\mathbf{x}}^{t+1}+\hat{\gamma}^{t+1}-\mathbf{d}^{t}-\mathbf{x}^{t}-\gamma^{t}\right\|^{2} \leq D$ for some $D>0$. It follows that

$$
\begin{aligned}
\mathcal{F}\left(\mathbf{x}^{t+1}, \gamma^{t+1}, \mathbf{d}^{t+1}\right) \geq & \mathcal{F}\left(\mathbf{x}^{t}, \gamma^{t}, \mathbf{d}^{t}\right)+\alpha_{t} \sum_{i}\left(\hat{d}_{i}^{t+1}-d_{i}^{t}\right) v_{i}^{\prime}\left(d_{i}^{t}\right) \\
& -\alpha_{t} \sum_{i}\left(\hat{x}_{i}^{t+1}-x_{i}^{t}\right) \cdot\left[C_{i}^{\prime}\left(x_{i}^{t}\right)+\frac{\partial C_{i}^{E}\left(\gamma_{i}^{t}, x_{i}^{t}\right)}{\partial x_{i}}\right]
\end{aligned}
$$




$$
\begin{aligned}
&-\alpha_{t} \sum_{i}\left(\hat{\gamma}_{i}^{t+1}-\gamma_{i}^{t}\right) \cdot \frac{\partial C_{i}^{E}\left(\gamma_{i}^{t}, x_{i}^{t}\right)}{\partial \gamma_{i}}-\frac{\alpha_{t}^{2}}{2} L D \\
&= \mathcal{F}\left(\mathbf{x}^{t}, \gamma^{t}, \mathbf{d}^{t}\right)+\alpha_{t} \sum_{i}\left[u_{i}^{t+1} \hat{d}_{i}^{t+1}-\left(c_{i}^{t+1}-\xi_{x, i}^{t}\right) \hat{x}_{i}^{t+1}-\left(\bar{c}_{i}^{t+1}-\xi_{\gamma, i}^{t}\right) \hat{\gamma}_{i}^{t+1}\right] \\
&-\alpha_{t} \sum_{i}\left[u_{i}^{t+1} d_{i}^{t}-\left(c_{i}^{t+1}-\xi_{x, i}^{t}\right) x_{i}^{t}-\left(\bar{c}_{i}^{t+1}-\xi_{\gamma, i}^{t}\right) \gamma_{i}^{t}\right]-\frac{\alpha_{t}^{2}}{2} L D \\
& \geq \mathcal{F}\left(\mathbf{x}^{t}, \gamma^{t}, \mathbf{d}^{t}\right)+\alpha_{t} \sum_{i}\left[\xi_{x, i}^{t}\left(\hat{x}_{i}^{t+1}-x_{i}^{t}\right)+\xi_{\gamma, i}^{t}\left(\hat{\gamma}_{i}^{t+1}-\gamma_{i}^{t}\right)\right]-\frac{\alpha_{t}^{2}}{2} L D
\end{aligned}
$$

where the last inequality is due to the fact that $\left(\hat{\mathbf{x}}^{t+1}, \hat{\gamma}^{t+1}, \hat{\mathbf{d}}^{t+1}\right)$ is an optimal solution to (5.14) for which $\left(\mathbf{x}^{t}, \gamma^{t}, \mathbf{d}^{t}\right)$ is a feasible solution.

Recalling that $\sum_{t} \alpha_{t}^{2}<\infty,\left\{\alpha_{t} \sum_{i}\left[\xi_{x, i}^{t}\left(\hat{x}_{i}^{t+1}-x_{i}^{t}\right)+\xi_{\gamma, i}^{t}\left(\hat{\gamma}_{i}^{t+1}-\gamma_{i}^{t}\right)\right]: t \geq 0\right\}$ is a sequence of zero mean independent random variables with finite total variance. Then

$$
\sum_{t} \alpha_{t} \sum_{i}\left[\xi_{x, i}^{t}\left(\hat{x}_{i}^{t+1}-x_{i}^{t}\right)+\xi_{\gamma, i}^{t}\left(\hat{\gamma}_{i}^{t+1}-\gamma_{i}^{t}\right)\right]
$$

converges almost surely (see Williams [105]). Since $\left(\mathbf{x}^{t}, \mathbf{d}^{t}\right)$ is bounded and $\mathcal{F}\left(\mathbf{x}^{t}, \gamma^{t}, \mathbf{d}^{t}\right)$ has Lipschitz continuous gradient, $\mathcal{F}\left(\mathbf{x}^{t}, \gamma^{t}, \mathbf{d}^{t}\right)$ is also bounded. We conclude that sequence $\left\{\mathcal{F}\left(\mathbf{x}^{t}, \gamma^{t}, \mathbf{d}^{t}\right): t>0\right\}$ converges almost surely.

\subsubsection{Proof of Theorem 9}

Referring to (5.18) and (5.19), after $T$ rounds the total payment for demand aggregator $i$ is

$$
\begin{aligned}
\tau_{i}^{T}= & \tau_{i}^{0}-\sum_{t=0}^{T-1} \sum_{k \neq i}\left(d_{k}^{t+1}-d_{k}^{t}\right) u_{k}^{t+1}+\sum_{t=0}^{T-1} \sum_{k}\left(x_{k}^{t+1}-x_{k}^{t}\right) c_{k}^{t+1}+\sum_{t=0}^{T-1} \sum_{k}\left(\gamma_{k}^{t+1}-\gamma_{k}^{t}\right) \bar{c}_{k}^{t+1} \\
= & \tau_{i}^{0}-\sum_{t=0}^{T-1} \sum_{k \neq i}\left(d_{k}^{t+1}-d_{k}^{t}\right) v_{k}^{\prime}\left(d_{k}^{t}\right)+\sum_{t=0}^{T-1} \sum_{k}\left(x_{k}^{t+1}-x_{k}^{t}\right)\left[C_{k}^{\prime}\left(x_{k}^{t}\right)+\frac{\partial C_{i}^{E}\left(\gamma_{k}^{t}, x_{k}^{t}\right)}{\partial x_{k}}+\xi_{x, k}^{t}\right] \\
& +\sum_{t=0}^{T-1} \sum_{k}\left(\gamma_{k}^{t+1}-\gamma_{k}^{t}\right)\left[\frac{\partial C_{i}^{E}\left(\gamma_{k}^{t}, x_{k}^{t}\right)}{\partial \gamma_{k}}+\xi_{\gamma, k}^{t}\right],
\end{aligned}
$$

and the total compensation for generator $i$ is

$$
\pi_{i}^{T}=\pi_{i}^{0}+\sum_{t=0}^{T-1} \sum_{k}\left(d_{k}^{t+1}-d_{k}^{t}\right) u_{k}^{t+1}-\sum_{t=0}^{T-1} \sum_{k \neq i}\left(x_{k}^{t+1}-x_{k}^{t}\right) c_{k}^{t+1}-\sum_{t=0}^{T-1} \sum_{k \neq i}\left(\gamma_{k}^{t+1}-\gamma_{k}^{t}\right) \bar{c}_{k}^{t+1}
$$




$$
\begin{aligned}
= & \pi_{i}^{0}+\sum_{t=0}^{T-1} \sum_{k}\left(d_{k}^{t+1}-d_{k}^{t}\right) v_{k}^{\prime}\left(d_{k}^{t}\right)-\sum_{t=0}^{T-1} \sum_{k \neq i}\left(x_{k}^{t+1}-x_{k}^{t}\right)\left[C_{k}^{\prime}\left(x_{k}^{t}\right)+\frac{\partial C_{i}^{E}\left(\gamma_{k}^{t}, x_{k}^{t}\right)}{\partial x_{k}}+\xi_{x, k}^{t}\right] \\
& -\sum_{t=0}^{T-1} \sum_{k \neq i}\left(\gamma_{k}^{t+1}-\gamma_{k}^{t}\right)\left[\frac{\partial C_{i}^{E}\left(\gamma_{k}^{t}, x_{k}^{t}\right)}{\partial \gamma_{k}}+\xi_{\gamma, k}^{t}\right] .
\end{aligned}
$$

Notice that the noise term

$$
\sum_{t=0}^{T-1}\left(x_{k}^{t+1}-x_{k}^{t}\right) \xi_{x, k}^{t}+\sum_{t=0}^{T-1}\left(\gamma_{k}^{t+1}-\gamma_{k}^{t}\right) \xi_{\gamma, k}^{t}
$$

has mean zero. In light of Assumption $7, \tau_{i}^{T}$ satisfies

$$
\begin{array}{r}
\mathbb{E}_{\xi}\left[\tau_{i}^{T}\right] \geq \tau_{i}^{0}-\left(\sum_{k \neq i} v_{k}\left(d_{k}^{T}\right)-\sum_{k} C_{k}\left(x_{k}^{T}\right)-\sum_{k} C_{k}^{E}\left(\gamma_{k}^{T}, x_{k}^{T}\right)\right)+\sum_{k \neq i} v_{k}\left(d_{k}^{0}\right)-\sum_{k} C_{k}\left(x_{k}^{0}\right) \\
-\sum_{k} C_{k}^{E}\left(\gamma_{k}^{0}, x_{k}^{0}\right)-\frac{1}{2} L D M
\end{array}
$$

and

$$
\begin{array}{r}
\mathbb{E}_{\xi}\left[\tau_{i}^{T}\right] \leq \tau_{i}^{0}-\left(\sum_{k \neq i} v_{k}\left(d_{k}^{T}\right)-\sum_{k} C_{k}\left(x_{k}^{T}\right)-\sum_{k} C_{k}^{E}\left(\gamma_{k}^{T}, x_{k}^{T}\right)\right)+\sum_{k \neq i} v_{k}\left(d_{k}^{0}\right)-\sum_{k} C_{k}\left(x_{k}^{0}\right) \\
-\sum_{k} C_{k}^{E}\left(\gamma_{k}^{0}, x_{k}^{0}\right) .
\end{array}
$$

Analogously,

$$
\begin{array}{r}
\mathbb{E}_{\xi}\left[\pi_{i}^{T}\right] \geq \pi_{i}^{0}+\left(\sum_{k} v_{k}\left(d_{k}^{T}\right)-\sum_{k \neq i} C_{k}\left(x_{k}^{T}\right)-\sum_{k \neq i} C_{k}^{E}\left(\gamma_{k}^{T}, x_{k}^{T}\right)\right)-\sum_{k} v_{k}\left(d_{k}^{0}\right)+\sum_{k \neq i} C_{k}\left(x_{k}^{0}\right) \\
+\sum_{k \neq i} C_{k}^{E}\left(\gamma_{k}^{0}, x_{k}^{0}\right)
\end{array}
$$

and

$$
\begin{array}{r}
\mathbb{E}_{\xi}\left[\pi_{i}^{T}\right] \leq \pi_{i}^{0}+\left(\sum_{k} v_{k}\left(d_{k}^{T}\right)-\sum_{k \neq i} C_{k}\left(x_{k}^{T}\right)-\sum_{k \neq i} C_{k}^{E}\left(\gamma_{k}^{T}, x_{k}^{T}\right)\right)-\sum_{k} v_{k}\left(d_{k}^{0}\right)+\sum_{k \neq i} C_{k}\left(x_{k}^{0}\right) \\
+\sum_{k \neq i} C_{k}^{E}\left(\gamma_{k}^{0}, x_{k}^{0}\right)+\frac{1}{2} L D M
\end{array}
$$


Hence the expected net surplus for demand aggregator $i$ satisfies

$$
\begin{aligned}
\mathbb{E}_{\xi}\left[v_{i}\left(d_{i}^{T}\right)-\tau_{i}^{T}\right] \in\left[\mathcal{F}\left(\mathbf{x}^{T}, \gamma^{T}, \mathbf{d}^{T}\right)-\mathcal{F}\left(\mathbf{x}^{0}, \gamma^{0}, \mathbf{d}^{0}\right)+v_{i}\left(d_{i}^{0}\right)-\tau_{i}^{0},\right. \\
\left.\mathcal{F}\left(\mathbf{x}^{T}, \gamma^{T}, \mathbf{d}^{T}\right)-\mathcal{F}\left(\mathbf{x}^{0}, \gamma^{0}, \mathbf{d}^{0}\right)+v_{i}\left(d_{i}^{0}\right)-\tau_{i}^{0}+\frac{1}{2} L D M\right] .
\end{aligned}
$$

In the limit $T \rightarrow \infty$,

$$
\mathbb{E}_{\xi}\left[v_{i}\left(d_{i}^{T}\right)-\tau_{i}^{T}\right] \geq \mathcal{F}\left(\mathbf{x}^{*}, \gamma^{*}, \mathbf{d}^{*}\right)-\mathcal{F}\left(\mathbf{x}^{0}, \gamma^{0}, \mathbf{d}^{0}\right)+v_{i}\left(d_{i}^{0}\right)-\tau_{i}^{0} .
$$

Untruthful behavior either results in divergent solutions, in which case the expected net surplus for demand aggregator $i$ is null, or leads to a suboptimal solution say $\left(\tilde{\mathbf{x}}^{*}, \tilde{\gamma}^{*}, \tilde{\mathbf{d}}^{*}\right)$. In the latter situation,

$$
\begin{aligned}
\mathbb{E}_{\xi}\left[v_{i}\left(d_{i}^{T}\right)-\tau_{i}^{T}\right] & \geq \mathcal{F}\left(\mathbf{x}^{*}, \gamma^{*}, \mathbf{d}^{*}\right)-\mathcal{F}\left(\mathbf{x}^{0}, \gamma^{0}, \mathbf{d}^{0}\right)+v_{i}\left(d_{i}^{0}\right)-\tau_{i}^{0} \\
& \geq \mathcal{F}\left(\tilde{\mathbf{x}}^{*}, \tilde{\gamma}^{*}, \tilde{\mathbf{d}}^{*}\right)-\mathcal{F}\left(\mathbf{x}^{0}, \gamma^{0}, \mathbf{d}^{0}\right)+v_{i}\left(d_{i}^{0}\right)-\tau_{i}^{0} \\
& \geq \mathbb{E}_{\xi}\left[v_{i}\left(\tilde{d}_{i}^{T}\right)-\tilde{\tau}_{i}^{T}\right]-\frac{1}{2} L D M .
\end{aligned}
$$

We will show in Section 5.5.3 that $\mathbb{E}_{\xi}\left[v_{i}\left(d_{i}^{T}\right)-\tau_{i}^{T}\right]>0$ with proper choice of $\tau_{i}^{0}$. Therefore, it is $\delta^{M}$-optimal for demand aggregator $i$ to be truthful when all the other participants are truthful.

The net surplus for generator $i$ satisfies

$$
\begin{array}{r}
\mathbb{E}_{\xi}\left[\pi_{i}^{T}-C_{i}\left(x_{i}^{T}\right)-C_{i}^{E}\left(\gamma_{i}^{T}, x_{i}^{T}\right)\right] \in\left[\mathcal{F}\left(\mathbf{x}^{T}, \gamma^{T}, \mathbf{d}^{T}\right)-\mathcal{F}\left(\mathbf{x}^{0}, \gamma^{0}, \mathbf{d}^{0}\right)-C_{i}\left(x_{i}^{0}\right)-C_{i}^{E}\left(\gamma_{i}^{0}, x_{i}^{0}\right)+\pi_{i}^{0},\right. \\
\left.\mathcal{F}\left(\mathbf{x}^{T}, \gamma^{T}, \mathbf{d}^{T}\right)-\mathcal{F}\left(\mathbf{x}^{0}, \gamma^{0}, \mathbf{d}^{0}\right)-C_{i}\left(x_{i}^{0}\right)-C_{i}^{E}\left(\gamma_{i}^{0}, x_{i}^{0}\right)+\pi_{i}^{0}+\frac{1}{2} L D M\right] .
\end{array}
$$

As $T \rightarrow \infty$,

$$
\begin{aligned}
\mathbb{E}_{\xi}\left[\pi_{i}^{T}-C_{i}\left(x_{i}^{T}\right)-C_{i}^{E}\left(\gamma_{i}^{T}, x_{i}^{T}\right)\right] \geq & \mathcal{F}\left(\mathbf{x}^{*}, \gamma^{*}, \mathbf{d}^{*}\right)-\mathcal{F}\left(\mathbf{x}^{0}, \gamma^{0}, \mathbf{d}^{0}\right)-C_{i}\left(x_{i}^{0}\right)-C_{i}^{E}\left(\gamma_{i}^{0}, x_{i}^{0}\right)+\pi_{i}^{0} \\
& \geq \mathcal{F}\left(\tilde{\mathbf{x}}^{*}, \gamma^{*}, \tilde{\mathbf{d}}^{*}\right)-\mathcal{F}\left(\mathbf{x}^{0}, \gamma^{0}, \mathbf{d}^{0}\right)-C_{i}\left(x_{i}^{0}\right)-C_{i}^{E}\left(\gamma_{i}^{0}, x_{i}^{0}\right)+\pi_{i}^{0} \\
& \geq \mathbb{E}_{\xi}\left[\tilde{\pi}_{i}^{*}+\tilde{\pi}_{i}^{E}-C_{i}\left(\tilde{d}_{i}^{*}\right)-C_{i}^{E}\left(\tilde{\gamma}_{i}^{*}, \tilde{x}_{i}^{*}\right)\right]-\frac{1}{2} L D M .
\end{aligned}
$$

Hence it is also $\delta^{M}$-optimal for generator $i$ to be truthful when all the other participants are truthful. 


\section{CHAPTER 6}

\section{Conclusions}

In this dissertation, we analyzed several multi-agent control/optimization algorithms for various problems under uncertainty. We now summarize them with a discussion on future work.

\subsection{Summary of the Dissertation}

Firstly, we developed a mathematical model for analyzing the benefits of social foraging in a noisy environment. We identified conditions on the nutrient profile ensuring that local agent actions will lead to cohesive foraging. For convex, smooth nutrient profiles we formalized the way in which swarming for social foraging is better at handling the effects of noise when compared to the average of individual foraging strategies. Under a swarming discipline, observational noise realizations that induce trajectories differing too much from the group average are likely to be discarded because of each individual's need to maintain cohesion. As a result, the group trajectories are less affected by noise. Simulation experiments indicate our theoretical results are robust to inter-agent communication constraints and non-convex nutrient profiles. These results suggest that swarming-like approaches for the control and/or optimization of networked agents may provide an additional level of robustness.

Secondly, inspired by the noise reduction property of swarming in social foraging, we proposed a distributed computing algorithmic scheme with $N>1$ computing threads operating under a connected network. At each step, each thread independently computes a 
new solution by using a noisy estimation of the gradient which is further perturbed by a combination of repulsive and attractive terms depending upon the relative distance to solutions identified by neighboring threads. This scheme resembles the mathematical models of flocking, swarming and other group formations found in nature. When the objective function is convex, we showed that a flocking-like approach for distributed stochastic optimization provides a noise reduction effect similar to that of a centralized stochastic gradient algorithm based upon the average of $N$ gradient samples at each step. When the overhead related to the time needed to gather $N$ samples and synchronization is not negligible, the flocking implementation outperforms a centralized stochastic gradient algorithm based upon the average of $N$ gradient samples at each step. When the objective function is not convex, the flocking-based approach seems better suited to escape locally optimal solutions due to the repulsive force which enforces a certain level of diversity in the set of candidate solutions. Here again, we showed that the noise reduction effect is similar to that associated to the centralized stochastic gradient algorithm based upon the average of $N$ gradient samples at each step.

Lastly, we proposed a class of iterative mechanisms for electricity markets. In an iterative mechanism, market participants are asked to report the cost or willingness to pay for small adjustments at each iteration and a tentative market dispatch is marginally adjusted ensuring network constraints are satisfied. Small adjustments require less information from market participants at each iteration and this facilitates the identification of incentives for ensuring truthful reporting of private information. At each iteration, each market participant is charged (or compensated) according to the marginal change in surplus to all other market participants. We show that truthful reporting is (approximately) a dominant strategy and that when the mechanism stops, the resulting economic dispatch is approximately efficient. Individual participation constraints are satisfied as the net surplus from trade is approximately shared equally by all participants and the mechanism is approximately weakly budget balanced. 


\subsection{Future Directions}

There remains several interesting and challenging directions for future research.

In the foraging model, we were assuming that the measurement of gradient is continuous and subject to i.i.d white noises. It would be interesting to explore the situation in which gradient signals are intermittent, and/or the random effects are state-dependent. These are practical concerns in both nature and engineering applications, e.g., when the signal of a nutrient profile is transmitted through turbulent fluids. In such complex environments, we may expect the repulsion force to play a crucial role in the foraging success.

In the presented flocking-type optimization algorithm, only constant stepsizes and fixed attraction/repulsion functions were considered. An important research direction is to design adaptive algorithms where stepsizes and attraction/repulsion functions vary in time depending upon the solutions obtained. This is crucial for ensuring convergence to the optimal solution when the variance of noise is great and when the objective function is potentially non-convex. Some work has been done in this respect on single-thread algorithms utilizing adaptive stepsizes (see [111] for instance) that may offer reference for improving the flocking-type algorithm.

The proposed iterative mechanisms for electricity markets may potentially be suitably altered in various ways as its main properties are not dependent upon the closed-form representation of network constraints but rather on convexity of the set of feasible solutions. Thus, the proposed mechanism may potentially be used in conjunction with convex approximations to $\mathrm{AC}$ power flow. Additionally, to speed up convergence, adjustments may be computed on the basis of an augmented Lagrangian. 


\section{Bibliography}

[1] Shin Mi Ahn and Seung-Yeal Ha. Stochastic flocking dynamics of the cuckersmale model with multiplicative white noises. Journal of Mathematical Physics, 51(10):103301, 2010.

[2] Edward J Anderson, Pär Holmberg, and Andrew B Philpott. Mixed strategies in discriminatory divisible-good auctions. The RAND Journal of Economics, 44(1):1-32, 2013.

[3] Tucker Balch and Ronald C Arkin. Behavior-based formation control for multirobot teams. Robotics and Automation, IEEE Transactions on, 14(6):926-939, 1998.

[4] Michele Ballerini, Nicola Cabibbo, Raphael Candelier, Andrea Cavagna, Evaristo Cisbani, Irene Giardina, Vivien Lecomte, Alberto Orlandi, Giorgio Parisi, Andrea Procaccini, et al. Interaction ruling animal collective behavior depends on topological rather than metric distance: Evidence from a field study. Proceedings of the national academy of sciences, 105(4):1232-1237, 2008.

[5] Dirk Bergemann and Alessandro Pavan. Introduction to symposium on dynamic contracts and mechanism design. Journal of Economic Theory, 159:679-701, 2015.

[6] Daniel Bienstock, Michael Chertkov, and Sean Harnett. Chance-constrained optimal power flow: Risk-aware network control under uncertainty. SIAM Review, 56(3):461495, 2014. 
[7] M Reza Bonyadi and Zbigniew Michalewicz. Particle swarm optimization for single objective continuous space problems: a review. Evolutionary computation, 2016.

[8] Tilman Borgers, Roland Strausz, and Daniel Krahmer. An introduction to the theory of mechanism design. Oxford University Press, USA, 2015.

[9] Vivek S Borkar et al. Stochastic approximation. Cambridge Books, 2008.

[10] Francois Bouffard, Francisco D Galiana, and Antonio J Conejo. Market-clearing with stochastic security-part i: formulation. Power Systems, IEEE Transactions on, 20(4):1818-1826, 2005.

[11] CM Breder. Equations descriptive of fish schools and other animal aggregations. Ecology, 35(3):361-370, 1954.

[12] Nicholas Britton. Essential mathematical biology. Springer Science \& Business Media, 2012.

[13] Francesco Bullo, Jorge Cortés, and Sonia Martinez. Distributed control of robotic networks: a mathematical approach to motion coordination algorithms. Princeton University Press, 2009.

[14] Yongcan Cao, Wenwu Yu, Wei Ren, and Guanrong Chen. An overview of recent progress in the study of distributed multi-agent coordination. Industrial Informatics, IEEE Transactions on, 9(1):427-438, 2013.

[15] José A Carrillo, Massimo Fornasier, Jesús Rosado, and Giuseppe Toscani. Asymptotic flocking dynamics for the kinetic cucker-smale model. SIAM Journal on Mathematical Analysis, 42(1):218-236, 2010.

[16] Renato LG Cavalcante and Slawomir Stanczak. A distributed subgradient method for dynamic convex optimization problems under noisy information exchange. Selected Topics in Signal Processing, IEEE Journal of, 7(2):243-256, 2013. 
[17] Edward H Clarke. Multipart pricing of public goods. Public choice, 11(1):17-33, 1971.

[18] Maurice Clerc and James Kennedy. The particle swarm-explosion, stability, and convergence in a multidimensional complex space. Evolutionary Computation, IEEE Transactions on, 6(1):58-73, 2002.

[19] Iain D Couzin, Jens Krause, Richard James, Graeme D Ruxton, and Nigel R Franks. Collective memory and spatial sorting in animal groups. Journal of theoretical biology, 218(1):1-11, 2002.

[20] Felipe Cucker and Jiu-Gang Dong. Avoiding collisions in flocks. Automatic Control, IEEE Transactions on, 55(5):1238-1243, 2010.

[21] Felipe Cucker and Ernesto Mordecki. Flocking in noisy environments. Journal de mathématiques pures et appliquées, 89(3):278-296, 2008.

[22] Felipe Cucker and Steve Smale. Emergent behavior in flocks. Automatic Control, IEEE Transactions on, 52(5):852-862, 2007.

[23] Rick Durrett. Probability: theory and examples. Cambridge university press, 2010.

[24] Krishnamurthy Dvijotham and Konstantin Turitsyn. Construction of power flow feasibility sets. arXiv preprint arXiv:1506.07191, 2015.

[25] Leah Edelstein-Keshet. Mathematical models in biology, volume 46. Siam, 1988.

[26] Natalia Fabra, Nils-Henrik Fehr, and David Harbord. Designing electricity auctions. The RAND Journal of Economics, 37(1):23-46, 2006.

[27] Michael C Fu. Stochastic gradient estimation. Springer, 2015.

[28] Crispin W Gardiner et al. Handbook of stochastic methods, volume 3. Springer Berlin, 1985.

[29] Veysel Gazi. On lagrangian dynamics based modeling of swarm behavior. Physica D: Nonlinear Phenomena, 260:159-175, 2013. 
[30] Veysel Gazi and Kevin M Passino. Stability analysis of swarms. Automatic Control, IEEE Transactions on, 48(4):692-697, 2003.

[31] Veysel Gazi and Kevin M Passino. A class of attractions/repulsion functions for stable swarm aggregations. International Journal of Control, 77(18):1567-1579, 2004.

[32] Veysel Gazi and Kevin M Passino. Stability analysis of social foraging swarms. Systems, Man, and Cybernetics, Part B: Cybernetics, IEEE Transactions on, 34(1):539-557, 2004.

[33] Veysel Gazi and Kevin M Passino. Swarm stability and optimization, volume 1. Springer, 2011.

[34] Stuart Geman and Chii-Ruey Hwang. Diffusions for global optimization. SIAM Journal on Control and Optimization, 24(5):1031-1043, 1986.

[35] Saeed Ghadimi and Guanghui Lan. Stochastic first-and zeroth-order methods for nonconvex stochastic programming. SIAM Journal on Optimization, 23(4):2341-2368, 2013.

[36] Chris Godsil and Gordon F Royle. Algebraic graph theory, volume 207. Springer Science \& Business Media, 2013.

[37] Theodore Groves. Incentives in teams. Econometrica: Journal of the Econometric Society, pages 617-631, 1973.

[38] Daniel Grünbaum. Schooling as a strategy for taxis in a noisy environment. Evolutionary Ecology, 12(5):503-522, 1998.

[39] Seung-Yeal Ha, Kiseop Lee, and Doron Levy. Emergence of time-asymptotic flocking in a stochastic cucker-smale system. Communications in Mathematical Sciences, $7(2): 453-469,2009$.

[40] Mark D Hill and Michael R Marty. Amdahl's law in the multicore era. Computer, $(7): 33-38,2008$. 
[41] Pär Holmberg and David Newbery. The supply function equilibrium and its policy implications for wholesale electricity auctions. Utilities Policy, 18(4):209-226, 2010.

[42] Chen Hong and Kong Li. Swarm intelligence-based extremum seeking control. Expert Systems with Applications, 38(12):14852-14860, 2011.

[43] Jiaqiao Hu. Model-based stochastic search methods. In Handbook of Simulation Optimization, pages 319-340. Springer, 2015.

[44] Jiaqiao Hu, Michael C Fu, and Steven I Marcus. A model reference adaptive search method for global optimization. Operations Research, 55(3):549-568, 2007.

[45] Jean Jacod and Philip E Protter. Probability essentials. Springer Science \& Business Media, 2003.

[46] Ali Jadbabaie, Jie Lin, and A Stephen Morse. Coordination of groups of mobile autonomous agents using nearest neighbor rules. Automatic Control, IEEE Transactions on, 48(6):988-1001, 2003.

[47] Alejandro Jofré, Nicolas Figueroa, and Benjamin Heymann. Mechanism design and allocation algorithms for network markets with piece-wise linear costs and quadratic externalities. Technical report, IPAM Workshop in Optimization and Equilibrium in Energy Economics., 2016.

[48] Alfred E Kahn, Peter C Cramton, Robert H Porter, and Richard D Tabors. Uniform pricing or pay-as-bid pricing: a dilemma for california and beyond. The Electricity Journal, 14(6):70-79, 2001.

[49] Javad Khazaei, Golbon Zakeri, and Shmuel S Oren. Market clearing mechanisms under uncertainty. Technical report, Working Paper, Princeton University, University of Auckland, University of California at Berkeley, 2014.

[50] Jack Kiefer, Jacob Wolfowitz, et al. Stochastic estimation of the maximum of a regression function. The Annals of Mathematical Statistics, 23(3):462-466, 1952. 
[51] Jack PC Kleijnen. Design and analysis of simulation experiments, volume 20. Springer, 2008.

[52] Anton J Kleywegt, Alexander Shapiro, and Tito Homem-de Mello. The sample average approximation method for stochastic discrete optimization. SIAM Journal on Optimization, 12(2):479-502, 2002.

[53] Harold Kushner and G George Yin. Stochastic approximation and recursive algorithms and applications, volume 35. Springer Science \& Business Media, 2003.

[54] Naomi Ehrich Leonard and Edward Fiorelli. Virtual leaders, artificial potentials and coordinated control of groups. In Decision and Control, 2001. Proceedings of the 40th IEEE Conference on, volume 3, pages 2968-2973. IEEE, 2001.

[55] Bernard C Lesieutre and Ian A Hiskens. Convexity of the set of feasible injections and revenue adequacy in ftr markets. IEEE Transactions on Power Systems, 20(4):17901798, 2005.

[56] Wei Li. Stability analysis of swarms with general topology. Systems, Man, and Cybernetics, Part B: Cybernetics, IEEE Transactions on, 38(4):1084-1097, 2008.

[57] Wei Li and Mark W Spong. Stability of general coupled inertial agents. Automatic Control, IEEE Transactions on, 55(6):1411-1416, 2010.

[58] Wei Li and Mark W Spong. Analysis of flocking of cooperative multiple inertial agents via a geometric decomposition technique. Systems, Man, and Cybernetics: Systems, IEEE Transactions on, 44(12):1611-1623, 2014.

[59] Yanfei Liu and Kevin M Passino. Stable social foraging swarms in a noisy environment. Automatic Control, IEEE Transactions on, 49(1):30-44, 2004.

[60] Ilan Lobel and Asuman Ozdaglar. Distributed subgradient methods for convex optimization over random networks. Automatic Control, IEEE Transactions on, 56(6):1291-1306, 2011. 
[61] Ali Marjovi, João Gonçalo Nunes, Lino Marques, and Aníbal De Almeida. Multi-robot exploration and fire searching. In Intelligent Robots and Systems, 2009. IROS 2009. IEEE/RSJ International Conference on, pages 1929-1934. IEEE, 2009.

[62] Sonia Martinez, Jorge Cortes, and Francesco Bullo. Motion coordination with distributed information. Control Systems, IEEE, 27(4):75-88, 2007.

[63] John McMillan. Reinventing the bazaar: A natural history of markets. WW Norton \& Company, 2003.

[64] Alexander Mogilner and Leah Edelstein-Keshet. A non-local model for a swarm. Journal of Mathematical Biology, 38(6):534-570, 1999.

[65] Alexander Mogilner, Leah Edelstein-Keshet, Leah R Bent, and Athan Spiros. Mutual interactions, potentials, and individual distance in a social aggregation. Journal of mathematical biology, 47(4):353-389, 2003.

[66] Luc Moreau. Stability of multiagent systems with time-dependent communication links. Automatic Control, IEEE Transactions on, 50(2):169-182, 2005.

[67] James D Murray. Mathematical biology II: spatial models and biomedical applications. Springer, 2001.

[68] James D Murray. Mathematical biology I: an introduction. Springer, 2002.

[69] Arkadi Nemirovski, Anatoli Juditsky, Guanghui Lan, and Alexander Shapiro. Robust stochastic approximation approach to stochastic programming. SIAM Journal on Optimization, 19(4):1574-1609, 2009.

[70] Yurii Nesterov. Introductory lectures on convex optimization: A basic course, volume 87. Springer Science \& Business Media, 2013.

[71] Bernt Øksendal. Stochastic differential equations. Springer, 2003. 
[72] Akira Okubo. Dynamical aspects of animal grouping: swarms, schools, flocks, and herds. Advances in biophysics, 22:1-94, 1986.

[73] Reza Olfati-Saber. Flocking for multi-agent dynamic systems: Algorithms and theory. Automatic Control, IEEE Transactions on, 51(3):401-420, 2006.

[74] Julia K Parrish and William M Hamner. Animal groups in three dimensions: how species aggregate. Cambridge University Press, 1997.

[75] Julia K Parrish, Steven V Viscido, and Daniel Grünbaum. Self-organized fish schools: an examination of emergent properties. The biological bulletin, 202(3):296-305, 2002.

[76] Kevin M Passino. Biomimicry of bacterial foraging for distributed optimization and control. Control Systems, IEEE, 22(3):52-67, 2002.

[77] Boris T Polyak and Anatoli B Juditsky. Acceleration of stochastic approximation by averaging. SIAM Journal on Control and Optimization, 30(4):838-855, 1992.

[78] Geoffrey Pritchard, Golbon Zakeri, and Andrew Philpott. A single-settlement, energyonly electric power market for unpredictable and intermittent participants. Operations research, 58(4-part-2):1210-1219, 2010.

[79] Shi $\mathrm{Pu}$, Alfredo Garcia, and Zongli Lin. Noise reduction by swarming in social foraging. Automatic Control, IEEE Transactions on, 2016.

[80] Craig W Reynolds. Flocks, herds and schools: A distributed behavioral model. In ACM SIGGRAPH computer graphics, volume 21, pages 25-34. ACM, 1987.

[81] Hannes Risken. Fokker-planck equation. Springer, 1984.

[82] Herbert Robbins and Sutton Monro. A stochastic approximation method. The annals of mathematical statistics, pages 400-407, 1951.

[83] Raina Robeva and Terrell Hodge. Mathematical concepts and methods in modern biology: using modern discrete models. Academic Press, 2013. 
[84] Andrija T Saric and Aleksandar M Stankovic. Applications of ellipsoidal approximations to polyhedral sets in power system optimization. Power Systems, IEEE Transactions on, 23(3):956-965, 2008.

[85] Alexander Shapiro. Monte carlo sampling methods. Handbooks in operations research and management science, 10:353-425, 2003.

[86] Yuhui Shi and Russell C Eberhart. Parameter selection in particle swarm optimization. In Evolutionary programming VII, pages 591-600. Springer, 1998.

[87] Carlos Silva, Bruce F Wollenberg, and Charles Z Zheng. Application of mechanism design to electric power markets. Power Systems, IEEE Transactions on, 16(1):1-8, 2001.

[88] James C Spall. Multivariate stochastic approximation using a simultaneous perturbation gradient approximation. Automatic Control, IEEE Transactions on, 37(3):332341, 1992.

[89] James C Spall. Introduction to stochastic search and optimization: estimation, simulation, and control, volume 65. John Wiley \& Sons, 2005.

[90] Kunal Srivastava and Angelia Nedić. Distributed asynchronous constrained stochastic optimization. Selected Topics in Signal Processing, IEEE Journal of, 5(4):772-790, 2011.

[91] Miloš S Stanković and Dušan M Stipanović. Extremum seeking under stochastic noise and applications to mobile sensors. Automatica, 46(8):1243-1251, 2010.

[92] Daniel Strömbom. Collective motion from local attraction. Journal of theoretical biology, 283(1):145-151, 2011.

[93] Housheng Su, Xiaofan Wang, and Zongli Lin. Flocking of multi-agents with a virtual leader. Automatic Control, IEEE Transactions on, 54(2):293-307, 2009. 
[94] Ichiro Suzuki and Masafumi Yamashita. Distributed anonymous mobile robots: Formation of geometric patterns. SIAM Journal on Computing, 28(4):1347-1363, 1999.

[95] Nicolas Tabareau, Jean-Jacques Slotine, and Quang-Cuong Pham. How synchronization protects from noise. PLoS Comput Biol, 6(1):e1000637, 2010.

[96] Herbert G Tanner, Ali Jadbabaie, and George J Pappas. Flocking in fixed and switching networks. Automatic Control, IEEE Transactions on, 52(5):863-868, 2007.

[97] John Toner and Yuhai Tu. Flocks, herds, and schools: A quantitative theory of flocking. Phys. Rev. E, 58:4828-4858, Oct 1998.

[98] Zaid J Towfic and Ali H Sayed. Adaptive penalty-based distributed stochastic convex optimization. Signal Processing, IEEE Transactions on, 62(15):3924-3938, 2014.

[99] Hal R Varian. Non-parametric analysis of optimizing behavior with measurement error. Journal of Econometrics, 30(1-2):445-458, 1985.

[100] Tamás Vicsek, András Czirók, Eshel Ben-Jacob, Inon Cohen, and Ofer Shochet. Novel type of phase transition in a system of self-driven particles. Physical review letters, $75(6): 1226,1995$.

[101] Tamás Vicsek and Anna Zafeiris. Collective motion. Physics Reports, 517(3):71-140, 2012.

[102] Irene K Voellmy, Julia Purser, Douglas Flynn, Philippa Kennedy, Stephen D Simpson, and Andrew N Radford. Acoustic noise reduces foraging success in two sympatric fish species via different mechanisms. Animal Behaviour, 89:191-198, 2014.

[103] Matthew A Wale, Stephen D Simpson, and Andrew N Radford. Noise negatively affects foraging and antipredator behaviour in shore crabs. Animal Behaviour, 86(1):111-118, 2013. 
[104] Huiwei Wang, Xiaofeng Liao, Tingwen Huang, and Chaojie Li. Cooperative distributed optimization in multiagent networks with delays. Systems, Man, and Cybernetics: Systems, IEEE Transactions on, 45(2):363-369, 2015.

[105] David Williams. Probability with martingales. Cambridge university press, 1991.

[106] Robert Wilson. rgame theoretic approaches to trading processessin tru (man bewley, ed., advances in economic theory: Fifth world congress, 1987.

[107] Steven Wong and J David Fuller. Pricing energy and reserves using stochastic optimization in an alternative electricity market. Power Systems, IEEE Transactions on, 22(2):631-638, 2007.

[108] Wencen Wu, Dongsik Chang, and Fumin Zhang. A bio-inspired robust 3d plume tracking strategy using mobile sensor networks. In Decision and Control (CDC), 2013 IEEE 52nd Annual Conference on, pages 4571-4578. IEEE, 2013.

[109] Wencen Wu and Fumin Zhang. Robust cooperative exploration with a switching strategy. Robotics, IEEE Transactions on, 28(4):828-839, 2012.

[110] Yunjian Xu and Steven H Low. An efficient and incentive compatible mechanism for wholesale electricity markets.

[111] Farzad Yousefian, Angelia Nedić, and Uday V Shanbhag. On stochastic gradient and subgradient methods with adaptive steplength sequences. Automatica, 48(1):56-67, 2012.

[112] Wenwu Yu, Guanrong Chen, and Ming Cao. Distributed leader-follower flocking control for multi-agent dynamical systems with time-varying velocities. Systems $\&$ Control Letters, 59(9):543-552, 2010.

[113] Jinye Zhao, Tongxin Zheng, and Eugene Litvinov. Variable resource dispatch through do-not-exceed limit. Power Systems, IEEE Transactions on, 30(2):820-828, 2015. 
[114] Rui Zou, Vijay Kalivarapu, Eliot Winer, James Oliver, and Sourabh Bhattacharya. Particle swarm optimization based source seeking. arXiv preprint arXiv:1501.06622, 2015. 